\title{
Phase-Coded-Linear-Frequency- Modulated Waveform for a Low Cost High Resolution Radar System
}

\author{
by Melin Ngwar \\ A thesis \\ presented to the Department of Electronics \\ in fulfillment of the \\ thesis requirement for the degree of \\ Masters of Applied Science \\ Ottawa-Carleton Institute for Electrical Engineering \\ Department of Electronics \\ Carleton University \\ Ottawa, Ontario
}


Library and Archives

Canada

Published Heritage

Branch

395 Wellington Street Ottawa ON K1A ON4 Canada
Bibliothèque et

Archives Canada

Direction du

Patrimoine de l'édition

395 , rue Wellington

Ottawa ON K1A ON4

Canada
Your file Votre référence

ISBN: 978-0-494-71567-3

Our file Notre référence

ISBN: 978-0-494-71567-3

\section{NOTICE:}

The author has granted a nonexclusive license allowing Library and Archives Canada to reproduce, publish, archive, preserve, conserve, communicate to the public by telecommunication or on the Internet, loan, distribute and sell theses worldwide, for commercial or noncommercial purposes, in microform, paper, electronic and/or any other formats.

The author retains copyright ownership and moral rights in this thesis. Neither the thesis nor substantial extracts from it may be printed or otherwise reproduced without the author's permission.
AVIS:

L'auteur a accordé une licence non exclusive permettant à la Bibliothèque et Archives Canada de reproduire, publier, archiver, sauvegarder, conserver, transmettre au public par télécommunication ou par l'Internet, prêter, distribuer et vendre des thèses partout dans le monde, à des fins commerciales ou autres, sur support microforme, papier, électronique et/ou autres formats.

L'auteur conserve la propriété du droit d'auteur et des droits moraux qui protège cette thèse. $\mathrm{Ni}$ la thèse ni des extraits substantiels de celle-ci ne doivent être imprimés ou autrement reproduits sans son autorisation.
In compliance with the Canadian Privacy Act some supporting forms may have been removed from this thesis.

While these forms may be included in the document page count, their removal does not represent any loss of content from the thesis.
Conformément à la loi canadienne sur la protection de la vie privée, quelques formulaires secondaires ont été enlevés de cette thèse.

Bien que ces formulaires aient inclus dans la pagination, il n'y aura aucun contenu manquant. 


\section{AUTHOR'S DECLARATION}

I hereby declare that I am the sole author of this thesis. This is a true copy of the thesis, including any required final revisions, as accepted by-my examiners.

I understand that my thesis may be made electronically available to the public. 


\begin{abstract}
Low cost Radar Systems are difficult to realize due to their high peak power requirement. High output power implies the need for unconventional and expensive power amplifiers such as magnetrons and travelling wave tubes. Peak output power is reduced by increasing the pulse width of the transmitted signal with minimum output power obtained from a continuous waveform or infinite pulse width. However, the drawback to increasing the pulse width is deterioration in resolution. This thesis uses pulse compression to achieve the required resolution. It combines two compression schemes: phase modulation and frequency modulation. Phase modulation is used to reject ambiguous targets while frequency modulation is used to achieve the finest resolution. Convolution of the phase modulated and frequency modulated waveforms yields a waveform which has the fine resolution property of the frequency modulated signal and the ambiguous target rejection property of the phase modulated signal. Target detection is done by correlation, and both types of pulse compression schemes have an auto-correlation response which approximates a delta function. Convolution is the only scheme which preserves the delta function auto-correlation response of the resulting waveform upon combination of the frequency and phase modulated signals. The Radar System demonstrated in this thesis also has a speed detection capability and this can be used to separate moving targets from stationery ones. Measured target speed is directly proportional to the frequency (Doppler) shift caused by moving targets and is obtained in this thesis by observing the phase shift over successive echoes. Since serial Doppler processing is done on correlated values, it has a higher CNR at the Doppler frequency than parallel Doppler processing performed on the received signal.
\end{abstract}




\section{Acknowledgements}

The guidance given by my supervisor, Professor Jim Wight has been-invaluable to my research efforts. His aid in the acquisition of Research grants and facilities has provided me with the best mental and physical environment throughout the course of this project.

All work done during the course of this research has been carried out at the D.Roy / T.Roy Advanced Sensor Processing Lab. I will like to acknowledge D-TA systems for financially supporting this research and providing the equipment required for experimental evaluation. I will also like to thank Pier Bortot, CTO of D-TA Systems for his invaluable assistance in all aspects of design and implementation.

Financial Support for this thesis was provided by the Department of Electronics at Carleton University, Faculty of Graduate Studies at Carleton University, Ontario Center of Excellence and D-TA Systems. 


\section{Table of Contents}

AUTHOR'S DECLARATION .................................................................................. ii

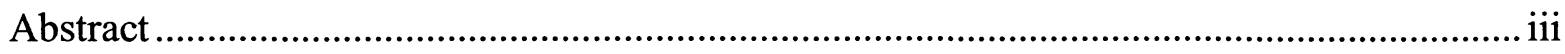

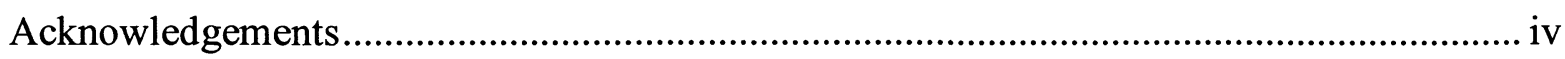

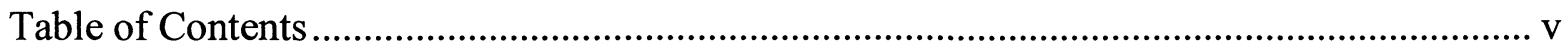

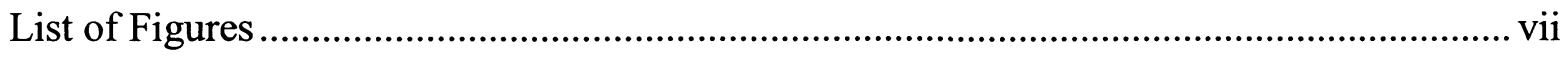

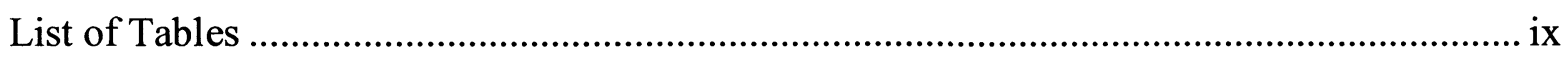

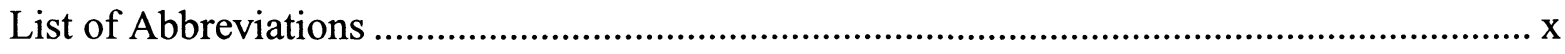

Chapter 1 Introduction to Low Cost Radar.................................................................. 1

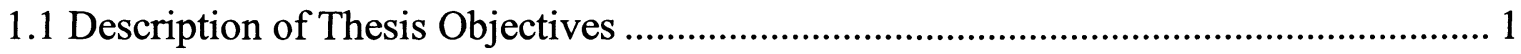

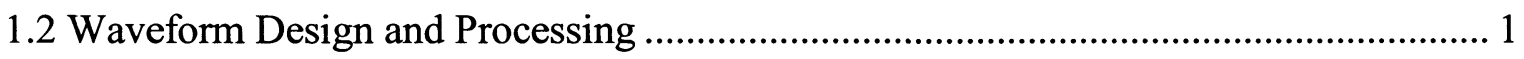

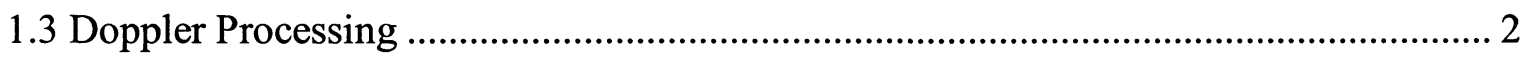

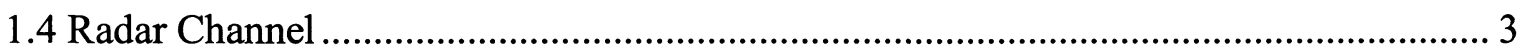

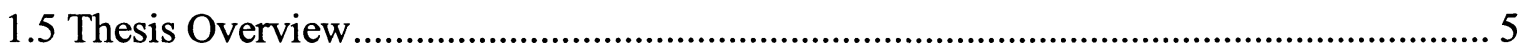

Chapter 2 Radar Overview and System Hardware ....................................................... 6

2.1 Background on Radar Concepts ...................................................................... 6

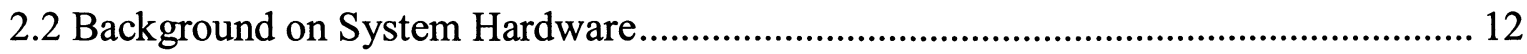

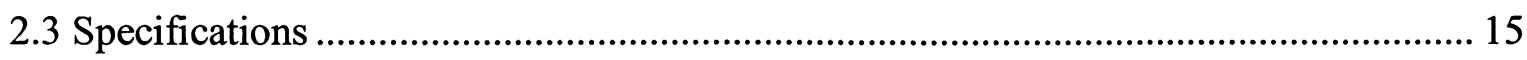

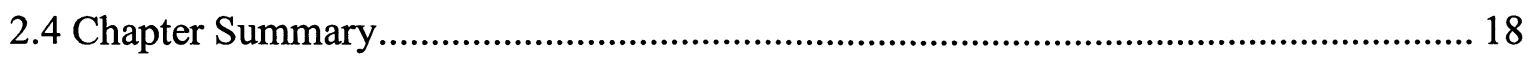

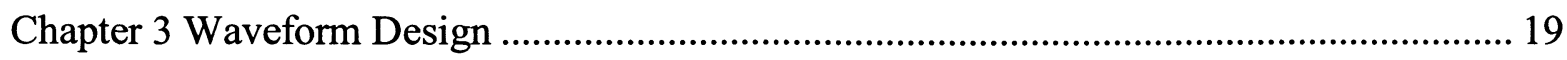

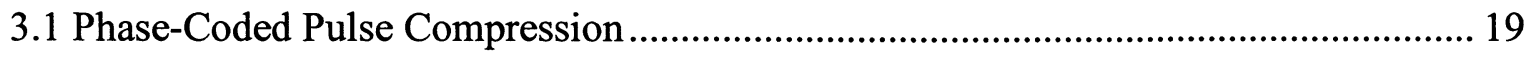

3.2 Frequency Modulation Compression ............................................................... 21 


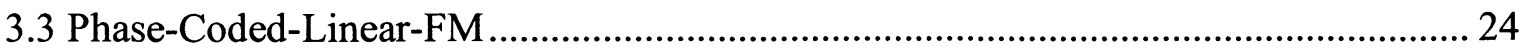

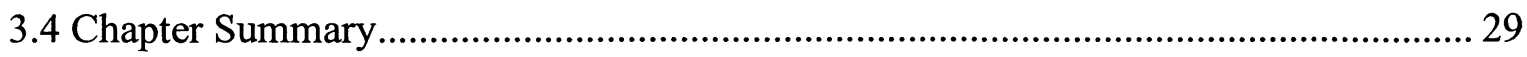

Chapter 4 Radar Simulation in Matlab ............................................................................ 30

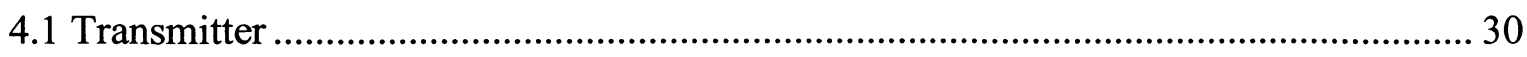

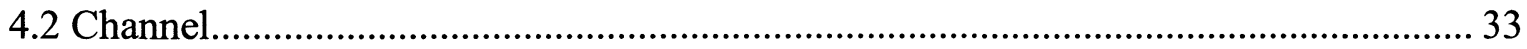

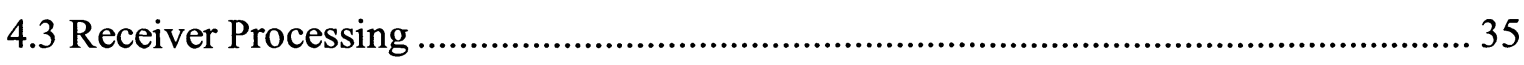

4.4 Simulated Serial Vs Parallel Processing ……………………...................................... 43

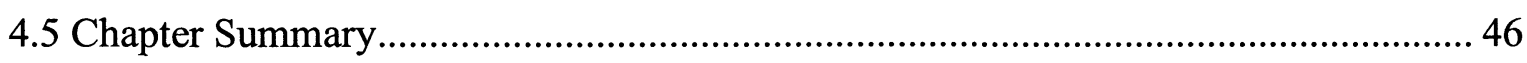

Chapter 5 Implementation and Testing ............................................................................... 47

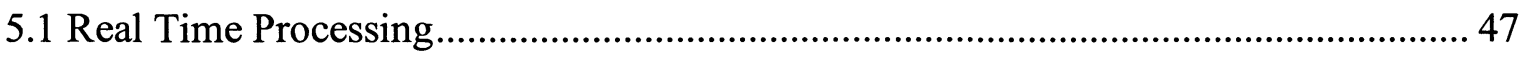

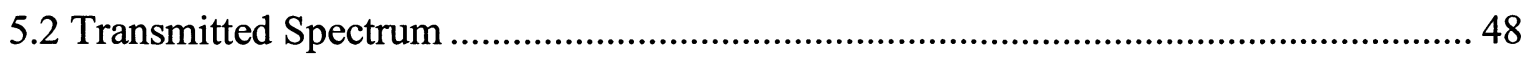

5.3 Resolution and Power Verification .............................................................................. 51

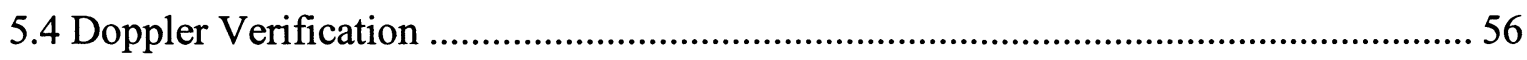

5.4.1 Doppler Test Setting ........................................................................................... 56

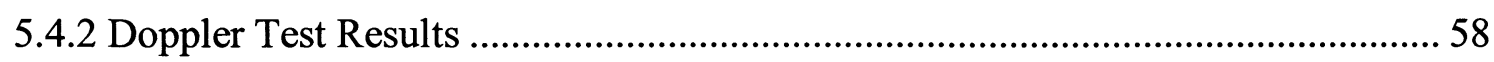

5.5 Tested Serial Vs Parallel Radar Processing ...................................................................... 61

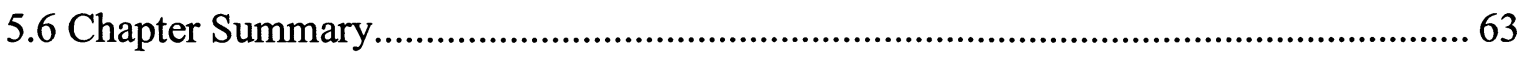

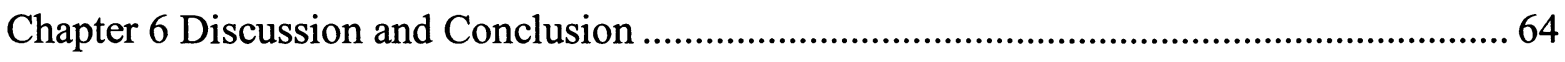

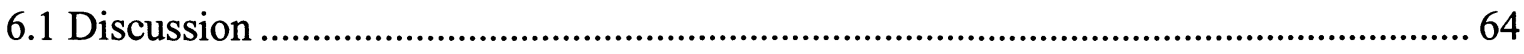

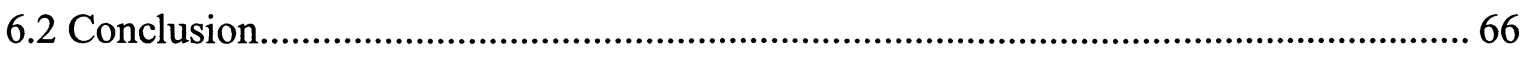

Appendix A Antenna Measurements ................................................................................... 70 


\section{List of Figures}

Figure 2.1: Generalized Receiver Chain ........................................................................... 9

Figure 2.2: Change in SNR with probability of Detection for various probabilities of false

alarm [4]

Figure 2.3: Probability distribution function for Swerling targets.......................................... 11

Figure 2.4: Plot of Additional SNR with Probability of detection for Swerling 1-4 [4]...... 12

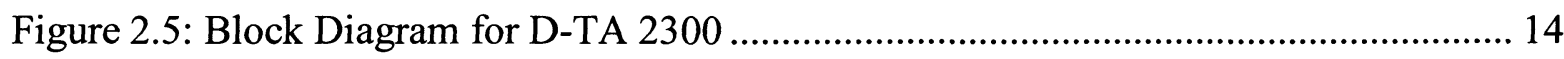

Figure 2.6: Block Diagram for D-TA 3290 (RF Up-converter) ............................................. 15

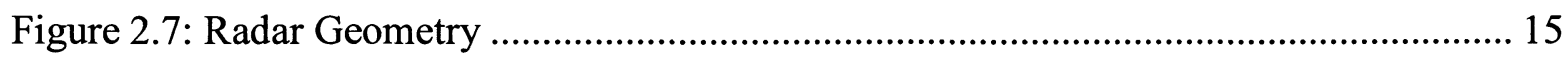

Figure 3.1: Auto-Correlation of MLS with $\mathrm{n}=20$ and $\mathrm{N}=1,048,575$ truncated to $640 \mathrm{bits}$ per

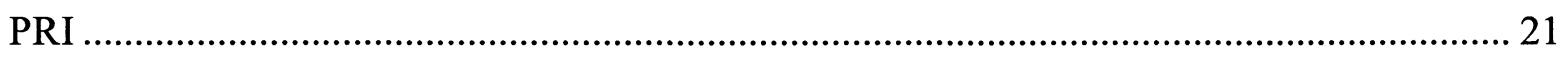

Figure 3.2: Auto-Correlation of linear-FM chirp................................................................ 24

Figure 3.3: Auto-Correlation of convolved phase modulated and linear-FM signal .............. 27

Figure 3.4: Normalized peak cross-correlation value over successive pulses ......................... 28

Figure 4.1: Block Diagram for the transmitter.................................................................. 31

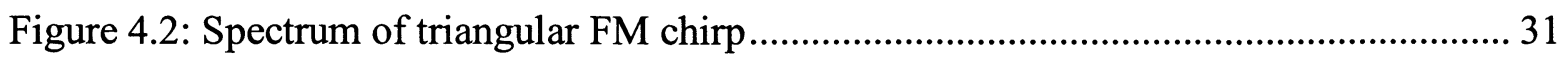

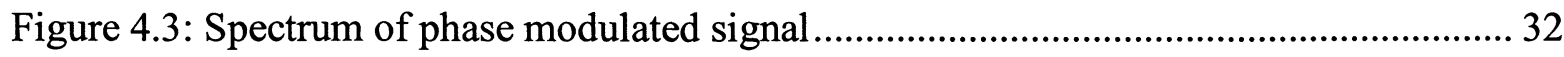

Figure 4.4: Spectrum of phase-coded-linear-frequency-modulated signal............................. 32

Figure 4.5: Block Diagram for Radar Channel .................................................................. 35

Figure 4.6: Block Diagram for Receiver Correlation Processing ............................................. 37

Figure 4.7: Simulated Auto-Correlation Response for a Swerling III or IV target ................. 37

Figure 4.8: Simulated Auto-Correlation Response for a Swerling I or II targets .................... 38 
Figure 4.9: Doppler Spectrum for Swerling I target ............................................................ 40

Figure 4.10: Doppler Spectrum for Swerling III target ....................................................... 40

Figure 4.11: Doppler Spectrum for Swerling II target.......................................................... 41

Figure 4.12: Doppler Spectrum for Swerling IV target .................................................... 41

Figure 4.13: Doppler Spectrum obtained from 1024 point FFT at clutter range bin............... 42

Figure 4.14: Doppler Spectrum of Serial process for non-fluctuating target .......................... 45

Figure 4.15: Doppler Spectrum of Parallel process for non-fluctuating target......................... 45

Figure 5.1: Block Diagram of Baseband Processor ................................................................ 48

Figure 5.2: Output of D-TA 2210 as seen on Spectrum Analyzer........................................ 49

Figure 5.3: Output of D-TA 3290 as seen on Spectrum Analyzer.......................................... 49

Figure 5.4: D-TA 2300 (on right) and RF transceiver (on left) ………………………....... 50

Figure 5.5: Circular polarized antenna (on left) and Horn antenna (on right) ........................ 50

Figure 5.6: Plot of Auto-correlation against range for phase-coded-linear-FM waveform ..... 53

Figure 5.7: Plot of Auto-correlation against range for phase-coded waveform........................ 54

Figure 5.8: Plot of Auto-correlation against range for linear-FM waveform ...........................5 54

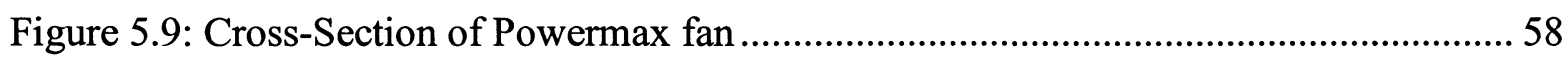

Figure 5.10: Doppler spectrum at target range bin for fan rotating at 1220rpm..................... 59

Figure 5.11: Doppler spectrum at target range bin for fan rotating at 1517rpm....................... 60

Figure 5.12: Serial and Parallel Doppler Spectrum of fan at 1220rpm .................................. 62

Figure 5.13: Serial and Parallel Doppler Spectrum of fan at 1517rpm ..................................62

Figure 6.1: Elevation plane Gain measurement for the $\mathrm{H}-1734$ antenna at $1.5 \mathrm{GHz}$.............. 70

Figure 6.2: Azimuth plane Gain measurement for the $\mathrm{H}-1734$ antenna at $1.5 \mathrm{GHz}$................ 70 


\section{List of Tables}

Table 5.1: Table of Normalized Auto-correlation values............................55

Table 5.2: Tested and Simulated Serial and Parallel carrier to noise ratios at different

frequencies..............................................................63 


\section{List of Abbreviations}

ADC Analog to Digital Converter

AWGN Additive White Gaussian Noise

CNR Carrier to Noise Ratio

DAC Digital to Analog Converter

dB decibels

FIFO First in First out buffer

FFT Fast Fourier Transform

FM Frequency Modulation

IF Intermediate Frequency

LFSR Linear Feedback Shift Register

LOS Line of Sight

MLS Maximum Length Sequence

NCO Numerically Controlled Oscillator

PLS Partial Length Sequence

PM Phase Modulation

PRI Pulse Repetition Interval

PRF Pulse Repetition Frequency 


\section{Chapter 1}

\section{Introduction to Low Cost Radar}

\subsection{Description of Thesis Objectives}

Most existing radar systems are quite expensive due to their high transmitter power requirement. Since transmission in pulsed radar only occurs for a small fraction of the PRI, a high power level is required to achieve a reasonable maximum range. Regular power amplifiers are insufficient to provide the required power levels, hence the need for TWTs and Magnetrons which are quite costly. The solution to this problem is to increase system gain by transmitting for $100 \%$ of the PRI or using a continuous waveform. In this way, the average power $\left(P_{a v}\right)$ can be maintained by decreasing the peak power $\left(P_{t}\right)$ and increasing the pulse width $(\tau)$ as derived from the equation 1.0.

$$
P_{a v}=\frac{P_{t} \tau}{P R I}
$$

The purpose of this thesis is to design and test a waveform which requires low power, has high resolution, rejects ambiguous targets and detects moving targets with a high carrier to noise ratio. The proposed waveform and processing is implemented on sensor interfaces built by D-TA Systems.

\subsection{Waveform Design and Processing}

Pulse compression is used to spread the transmitted energy and at the same time achieve the same resolution as a narrow pulse. The most common pulse compression schemes are phase-coded and frequency modulated waveforms. This thesis examines the said 
compression schemes and proposes a waveform which has both phase coding and frequency modulation properties. Range resolution is the minimum detectable separation between two targets at the same bearing. Both frequency and phase modulated waveforms have finer range resolution than pulsed waveforms for a particular bandwidth. However, the resolution of phase modulated signals is limited by the IF bandwidth while the optimum achievable resolution of a frequency modulated signal is limited by the sampling frequency. This implies that FM signals can achieve much finer resolution than phase modulated signals. FM signals are however, periodic meaning ambiguous targets are not rejected. Phase modulated signals are derived from truncated random sequences with long periods. The implication is PM signals can reject ambiguous targets by cross-correlation. The proposed combined scheme possesses the fine resolution property of the FM signal and ambiguous target rejection property of the PM signal. Target range is obtained by auto-correlation of the transmitted signal and its echo. Correlation compresses the echo such that even signals below the noise floor appear as a peak at the appropriate range bin.

\subsection{Doppler Processing}

For Doppler detection, typical radar systems detect the phase of the echo over successive pulses and use a bank of filters (FFT) to detect the Doppler frequency of the target. For echoes below the noise floor, a huge FFT length or number of filters is required to obtain the Doppler frequency. Doppler detection which is done by processing the auto-correlation phase is called serial Doppler processing because the range is first detected by auto-correlation before Doppler is processed. This way the FFT length required to detect the Doppler

frequency is much smaller and hence a more efficient use of resources. The drawback to 
system processing in this fashion is greater latency as range and Doppler processing are done sequentially as opposed to systems which process in parallel. Parallel Doppler processing refers to systems which detect the Doppler shift and range simultaneously. While parallel processing might be faster than serial processing, the resulting carrier to noise ratio at the Doppler frequency is often smaller than that obtained from a serial process. This is because serial processes extract the signal from the noise before Doppler processing while parallel processes do not.

\subsection{Radar Channel}

Accurate values of range for low angle targets are often difficult to determine for most radars due to the presence of multipath. In maritime environments most of this error is manifested in the elevation because the echo's image occurs only in elevation even though residual cross-talk can cause manifestation of this error in the azimuth [1]. With knowledge of the delay between the line of sight and multipath component, compression parameters can be chosen such that the line of sight and multipath components are separated by correlation. In this way the radar system acts as a Rake receiver without recombination as the radar receiver is only interested in the path of minimum delay. Poor elevation accuracy is quite detrimental to system performance because it limits the Radar's ability to implement effective countermeasures to impending threats. For example missiles approaching a ship travel at very high speed thus they should be detected with acceptable accuracy using the fewest number of pulses. The radar system discussed in this paper is tailored to operate in the presence of both land and sea clutter. Land clutter has a Doppler shift around zero while sea 
clutter comprises of waves with a non-zero Doppler shift. Sea clutter has a Doppler spectrum associated with it and its average Doppler shift is proportional to the wind velocity. Clutter Doppler shift is however a lot smaller than that of expected targets hence seaclutter can be distinguished from targets as such. The bandwidth of the sea clutter spectrum is much larger than that of land clutter and as a result some sea waves appear as targets. As a result, the Doppler cut-off for radar operation in the presence of sea clutter is much larger than that of land clutter.

Echoes at the receiver originating from a particular target are a result of line of sight and surface reflection. The difference between the earliest and latest multipath components for a particular environment is the delay spread. The deviation from the delay spread is often quite large because the multipath delay at any particular time depends on the target height and elevation. Lower elevation angles at any particular range have smaller multipath delays compared to higher elevation angles as shown in equation 2.13 . This makes their multipath components more difficult to separate from their line of sight. Also the further the target, the smaller the multipath delay for a particular elevation (see equation 2.13). Compressed pulses with chip length much greater that the delay-spread encounter flat fading or constructive multipath interference. The drawbacks to pulses with large chip lengths are poor resolution and an inability to separate individual multipath components. Radar systems which utilize compressed pulses designed to encounter flat fading are unable to accurately determine the elevation angle due to the multipath effect on monopulse antennas [2]. This implies that a system which can separate multipath from line of sight benefits from better range resolution and elevation accuracy. Such systems should be designed to operate at the unambiguous 
range for low angles of elevation and targets within the limits of operation will achieve superior performance.

\subsection{Thesis Overview}

Chapter 1 provides a description of the Radar problem and an overview of the Design methodology. Chapter 2 describes the Radar concepts utilized in the waveform design and processing. Chapter 2 also provides a background on the system hardware required for waveform implementation. Chapter 3 is a detailed examination of the Design process used to generate the proposed phase-coded-linear-FM signal. Chapter 4 shows the simulated performance of the proposed waveform under channel effects while Chapter 5 shows the tested results. Finally, Chapter 6 provides a comparison of the expected, simulated and tested results. 


\section{Chapter 2}

\section{Radar Overview and System Hardware}

\subsection{Background on Radar Concepts}

Radio Detection and Ranging (RADAR) is a system that determines the location of objects by transmitting a signal and detecting its echo. A portion of the transmitted signal that reaches the target is reflected in all directions and the radar antenna collects the returned energy in its direction [2]. The distance from Radar to target (R) as shown in equation 2.1 is determined by measuring the time between the transmitted signal and its echo reception $(\Delta t)$. $R=\frac{c \Delta t}{2}$

Where $\mathrm{c}$ is the speed of light. Target azimuth is obtained by rotating the antenna about a vertical axis while elevation is obtained by determining the angle of arrival from beam processing. Moving targets apply a frequency shift (Doppler effect) to the transmitted signal which is proportional to the relative velocity between the Radar and target. The receiver uses these frequency shifts to distinguish moving targets from stationery ones. The maximum expected (unambiguous) range determines the maximum pulse length or pulse repetition interval (PRI). The maximum pulse rate is the inverse of the PRI and is called the pulse repetition frequency (PRF). Echoes which take longer than the PRI to arrive represent ambiguous targets and they cause faulty range measurements if they aren't rejected, because the receiver doesn't know which transmitted pulse they correspond to. Unambiguous range is given by equation 2.2 . 
$R_{U N A M B I G}=\frac{c}{2 * P R F}$

Pulse compression utilizes frequency or phase modulation for compression of the transmitted signal. Longer pulses have higher energy and compression ensures that range resolution is not affected. Range resolution is given by equation 2.1 , where $\Delta t$ is the pulse width for rectangular pulses and the chip width in the case of phase modulated pulses. The maximum range is determined by the minimum signal the receiver can detect and is given by equation 2.3.

$R_{\text {max }}^{4}=\frac{P_{t} G_{t} G_{r} \sigma \lambda^{2}}{(4 \pi)^{2} P_{r_{-} \text {min }}}$

$P_{t}$ is peak transmitter power, $G_{t}$ and $G_{r}$ are peak transmitter and receiver gain respectively, $\sigma$ is the target cross-sectional area, $\lambda$ is the signal wavelength and $P_{r_{-} \min }$ is the minimum received power. $R_{\max }$ is limited by the minimum received power and could represent both ambiguous and unambiguous targets; processing is required to distinguish one from the other.

For a generalized receiver chain shown in figure 2.1 below, the signal envelope at the output of the matched filter with exclusively noise at its input has a Rayleigh probability distribution function provided that the channel is corrupted by Additive White Gaussian Noise. The Rayleigh PDF with variance of $\left(\psi_{o}\right)$ is shown in equation 2.4 below.

$p(R)=\frac{R}{\psi_{o}} \exp \left(-R^{2} / 2 \psi_{o}\right)$

The probability that a false alarm occurs becomes the probability that the noise voltage envelope exceeds a particular threshold $\left(V_{T}\right)$ and is given by equation 2.5 . 
$P_{f a}=\int_{V_{T}}^{\infty} p(R) d R=e^{\left(-V_{T}^{2} / 2 \psi_{o}\right)}$

If the signal at the receiver input consists of a sinusoid with amplitude $A$ and noise, the envelope at the output of the band-pass filter has a Rician probability distribution function shown in equation 2.6 .

$p(R)=\frac{R}{\psi_{o}} \exp \left(-\left(R^{2}+A^{2}\right) / 2 \psi_{o}\right) * I_{o}\left(R A / \psi_{o}\right)$

Where $I_{o}$ is the modified Bessel function of zero order.

The Probability that a target is detected is the probability that the signal plus noise envelope exceeds a given threshold as shown in equation 2.7.

$P_{d}=\frac{1}{2}\left[1-\operatorname{erf}\left(\frac{V_{T}-A}{\sqrt{2 \psi_{O}}}\right)\right]+\left[\exp \left(-\frac{\left(V_{T}-A\right)^{2} / 2 \psi_{O}}{2 \sqrt{2 \pi} \mathrm{A} / \sqrt{\psi_{O}}}\right)\right]\left[1-\left(\frac{V_{T}}{4 \mathrm{~A}}+\frac{1+\left(V_{T}-A\right)^{2} / \psi_{O}}{\left(8 \mathrm{~A}^{2}\right) / \psi_{O}}\right)\right]$

Substituting equation 2.5 and $\frac{A}{\sqrt{\psi_{O}}}=\sqrt{2 S N R}$ into the expression for probability of detection (2.7) yields the curves shown in figure 2.2. Figure 2.2 shows the variation of signal to noise ratio (SNR) with probability of detection $\left(P_{d}\right)$ for various values of probability of false alarm $\left(P_{f a}\right) . P_{f a}$ and $P_{d}$ are processing parameters which are usually initially known and figure 2.2 can be used to determine the required SNR for a single pulse return. Multiple pulse returns can be used to improve the resulting SNR by pulse integration. If the target cross-section is large compared to the beam-width, the number of pulses returned from a target during a single scan is given by equation 2.8 .

$n_{B}=\frac{\theta_{B} f_{P}}{\dot{\theta}_{s}}$ 
Where $\theta_{B}$ is the antenna beam-width in degrees, $f_{P}$ the PRF and $\dot{\theta}_{s}$ the antenna scan rate in $\operatorname{deg} / \mathrm{s}$.

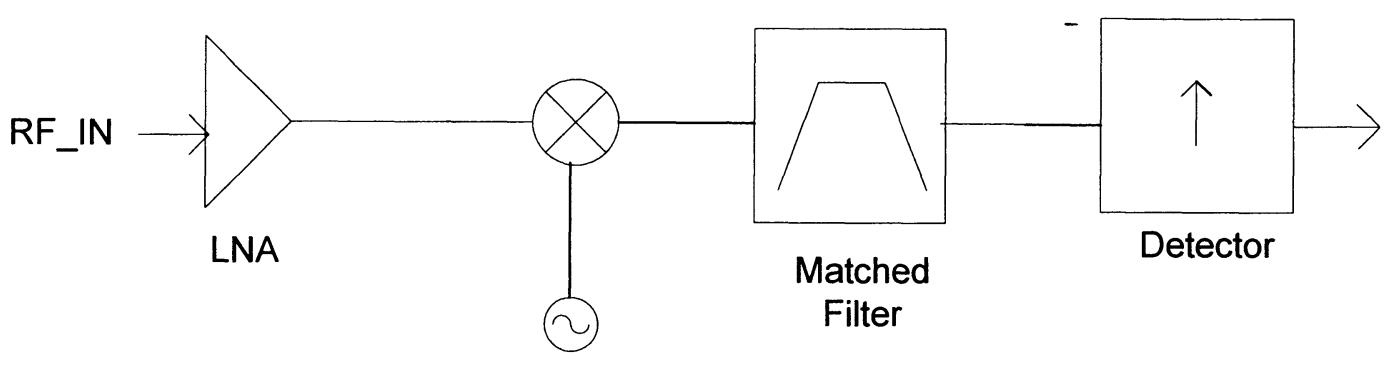

Figure 2.1: Generalized Receiver Chain

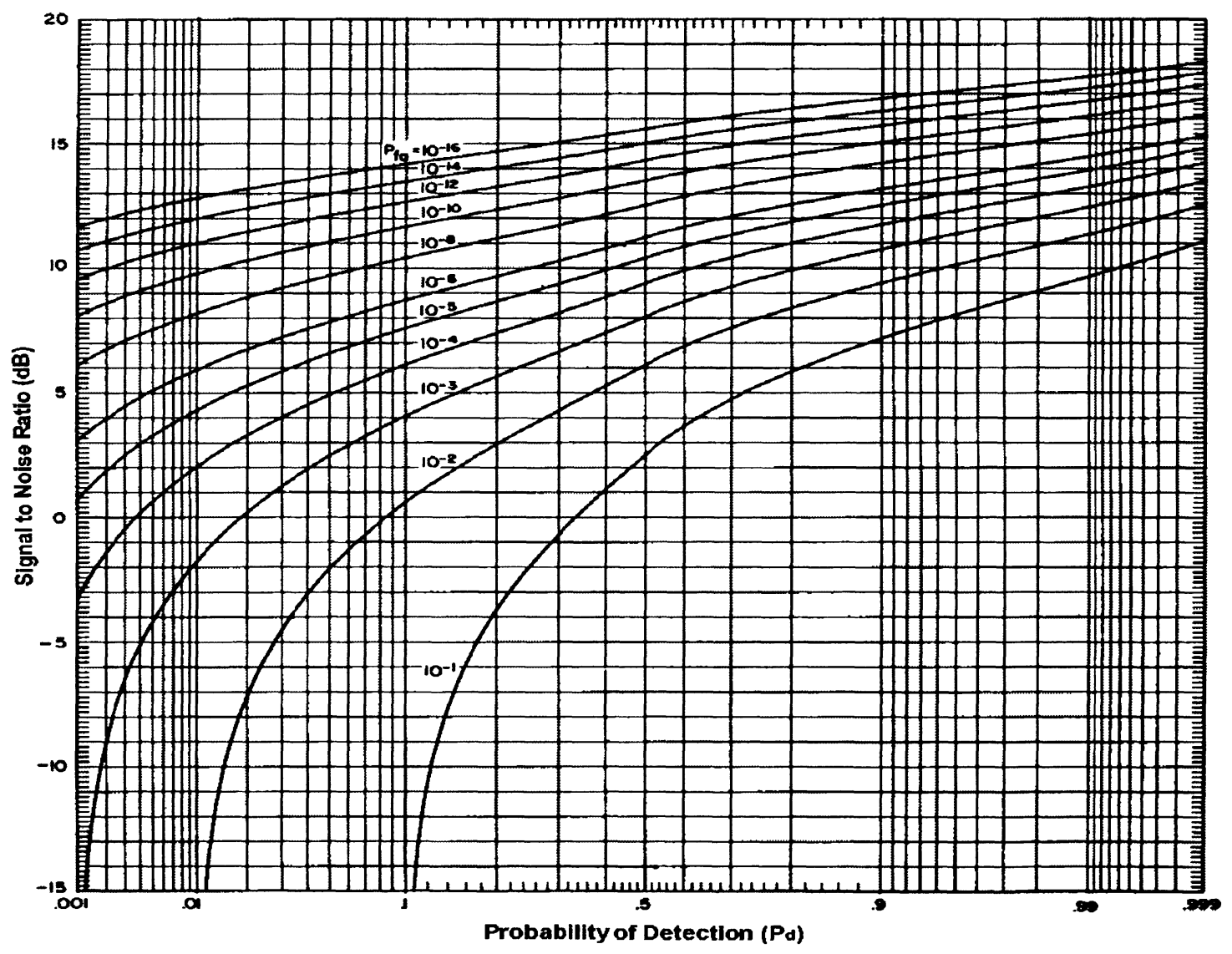

Figure 2.2: Change in SNR with probability of Detection for various probabilities of false alarm [4] 
The filter in figure 2.1 is matched to the transmitted signal hence yielding an optimum signal to noise ratio at its output. The detector at the filter output uses a pre-determined SNR threshold based on the $P_{d}$ and $P_{f a}$ to indicate the-presence of a target. Pre-detection integration and post-detection integration sum up the SNRs at the input and output of the detector respectively. For pre-detection integration: $S N R_{1}=n S N R_{n}$, where $S N R_{n}$ is the SNR of a single pulse while $S N R_{1}$ is the total SNR of the integrated pulses [5]. For postdetection integration:

$S N R_{1}=n E_{i}(n) S N R_{n}$, where $E_{i}(n)$ is the integration efficiency. $E_{i}(n)$ depends on the type of detector used and is usually experimentally determined. Equation 2.3 can be expressed in terms of $S N R_{1}$ by replacing the minimum received power with equation 2.9.

$P_{r_{-} \min }=k T_{o} B_{n} F_{n} S N R_{n}$

Where $F_{n}$ is the noise factor, $k$ is Boltzmann's constant, $T_{o}$ is room temperature and $B_{n}$ the noise bandwidth. Substituting equation 2.9 in 2.3 and replacing $S N R_{n}$ with $\frac{S N R_{1}}{n E_{i}(n)}$ yields equation 2.10.

$R_{\max }^{4}=\frac{P_{t} G^{2} \lambda^{2} \sigma n E_{i}(n)}{(4 \pi)^{3} k T_{o} B_{n} F_{n} S N R_{1}}$

Target cross-sections are classified into five Swerling categories according to their probability distribution function (pdf) and their pulse to pulse cross-section variation. The pdf for Swerling I and II is given in equation 2.11 and is shown in figure 2.3 for an average cross-section of $2 m^{2}$. 
$p(\sigma)=\frac{1}{\sigma_{\text {ave }}} \exp \left(-\sigma / \sigma_{\text {ave }}\right)$

For Swerling I, the cross-section is constant throughout the scan but independent from scan to scan while Swerling II has an independent cross-section from pulse to pulse. The pdf for Swerling III and IV is shown in equation 2.12 below and is also shown in figure 2.3 for an average cross-section of $4 m^{2}$.

$$
p(\sigma)=\frac{4 \sigma}{\sigma_{\text {ave }}} \exp \left(-2 \sigma / \sigma_{\text {ave }}\right)
$$

Again, for Swerling III, the cross-section is constant throughout the scan but independent from scan to scan while Swerling IV has an independent cross-section from pulse to pulse [4]. Due to target fluctuation, additional SNR to that required for a non-fluctuating target is required to achieve a specified probability of detection. Figure 2.4 shows the variation in additional SNR with probability of detection for all four Swerling cases.
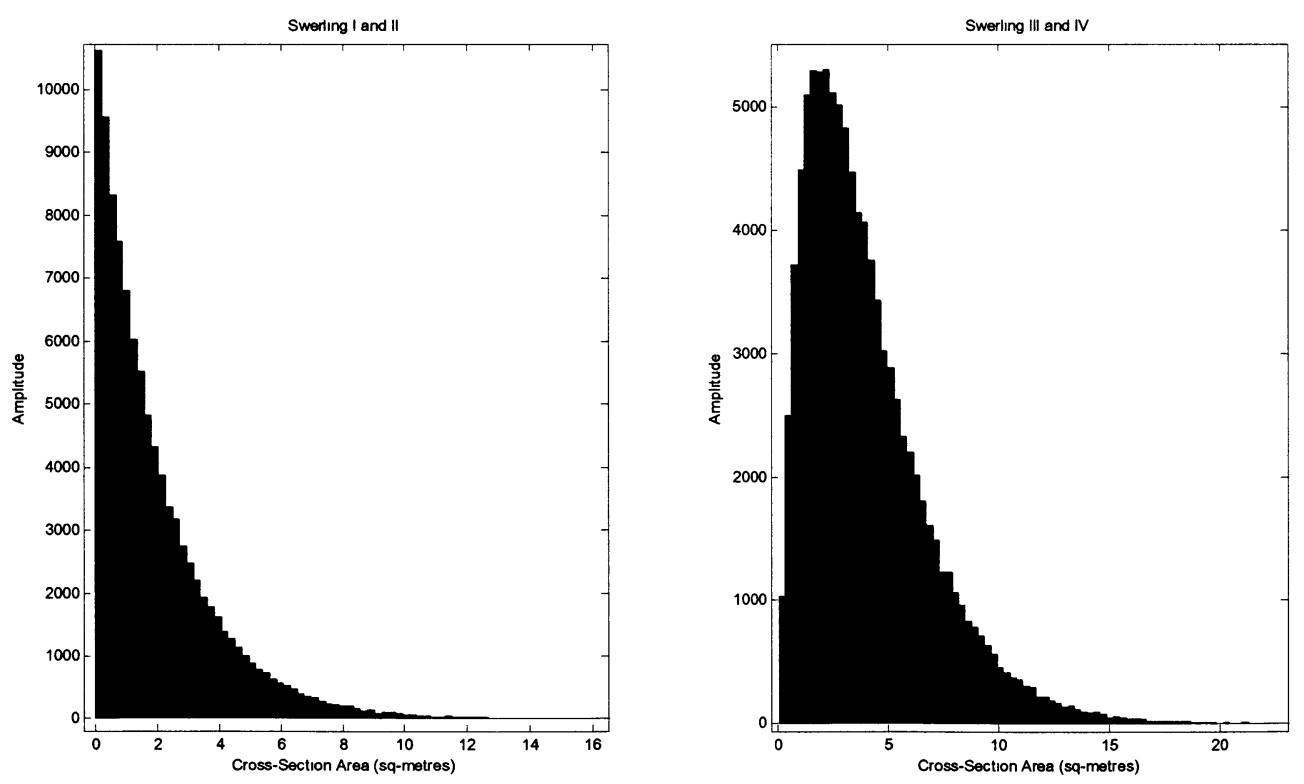

Figure 2.3: Probability distribution function for Swerling targets 


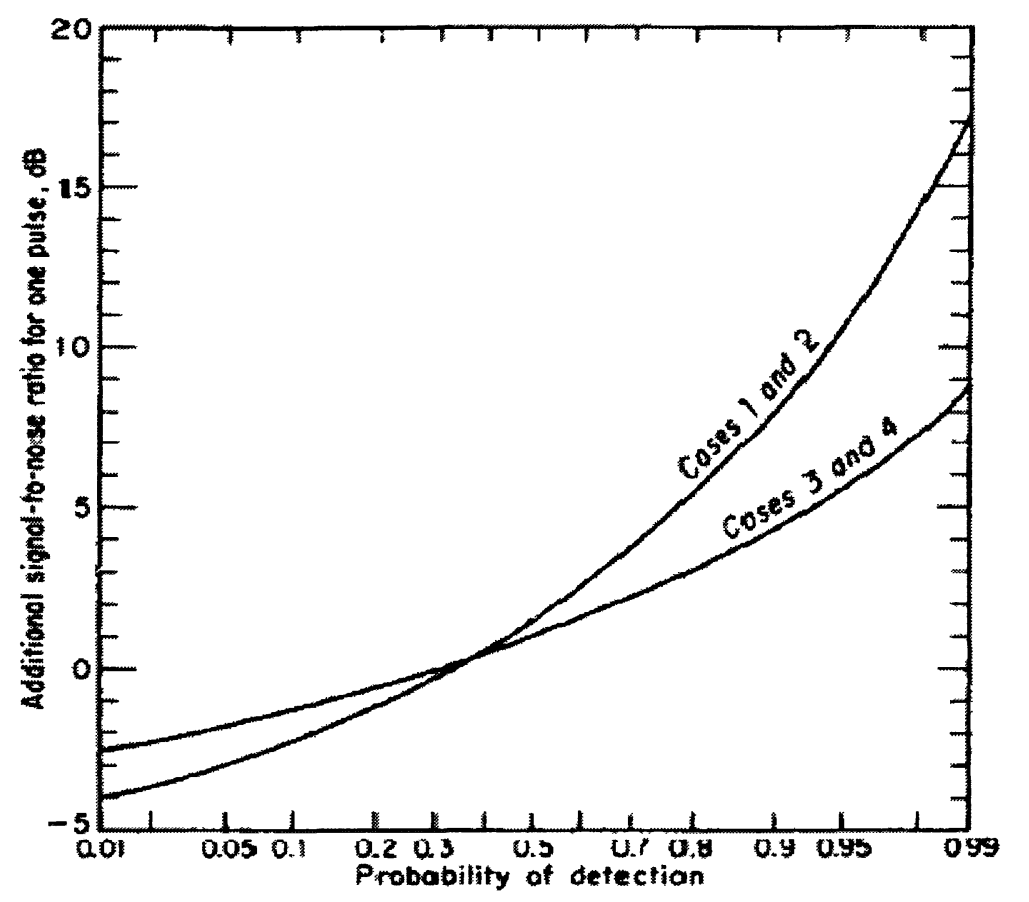

Figure 2.4: Plot of Additional SNR with Probability of detection for Swerling 1 - 4 [4]

\subsection{Background on System Hardware}

The hardware used to implement the Radar system in this thesis is designed by D-TA Systems and it is comprised of two software radio systems and a computer. The first software radio labeled D-TA 2300 receives a waveform in the form of packets from the computer and converts it to an analog signal. It also digitizes analog signals and groups them into packets in accordance with a user-specified packet size. Its DAC frequency is tunable; the maximum DAC frequency with interpolation is $500 \mathrm{MHz}$ and $130 \mathrm{MHz}$ without. This puts a $62.5 \mathrm{MHz}$ limit on the signal bandwidth and $250 \mathrm{MHz}$ limit on the center frequency. The maximum ADC sampling frequency is $130 \mathrm{MHz}$ and this places a $62.5 \mathrm{MHz}$ bandwidth restriction on the input signal. Figures 2.5 and 2.6 show block diagrams for the D-TA 2300 and D-TA 3290 
respectively. The D-TA 2300 stores data from the PC in a buffer (FIFO) for DAC sampling. The DAC chip possesses a programmable interpolator and an NCO for up-conversion. The maximum NCO frequency is half the DAC chip sampling frequency after interpolation. DAC chip sampling is obtained from the Phase Locked Loop shown in figure 2.5. The PLL operates off a $100 \mathrm{MHz}$ TCXO reference input and a VCO with range of $375 \mathrm{MHz}$ to $415 \mathrm{MHz}$ at its output. During transceiver operation, when both the ADC and DAC are in operation, their sampling clock is obtained from the same VCO. This implies that even though they may have different divider ratios, their base factor is always the same. Antenna sizes are inversely proportional to the carrier frequency implying the higher the carrier frequency, the smaller the antenna. To obtain a carrier frequency which yields a reasonable antenna size, a carrier frequency of at least $1 \mathrm{GHz}$ is required and to achieve this carrier, an RF system is needed. The D-TA 3290 accepts an input signal centered at $70 \mathrm{MHz}$ with a $40 \mathrm{MHz}$ bandwidth and upconverts it to any frequency in the 0 to $6 \mathrm{GHz}$ range. It also accepts an RF input in the 0 to $6 \mathrm{GHz}$ range and down-converts to $70 \mathrm{MHz}$ with $40 \mathrm{MHz}$ of bandwidth. The input and output IF filters are both centered at $70 \mathrm{MHz}$ with $40 \mathrm{MHz}$ of bandwidth. The D-TA 3290 utilizes 4 synthesizers in different combinations for up-conversion and down-conversion as depicted in the UCON and DCON circuitry respectively to achieve the required RF range. Both the UCON and DCON generate their respective outputs depending on the user-specified (programmable) RF and IF output frequencies. The filter bank at the mixer output for DCON and input for UCON consist of filters which select different frequency bands in the $0-6 \mathrm{GHz}$ range. Switches are placed at their inputs and outputs to select the appropriate frequency band. A power amplifier is the final link in the up-converter chain. The down-converter has 4 
Low Noise Amplifiers which cover the $0-6 \mathrm{GHz}$ band and a switch is used to select the appropriate low noise amplifier.

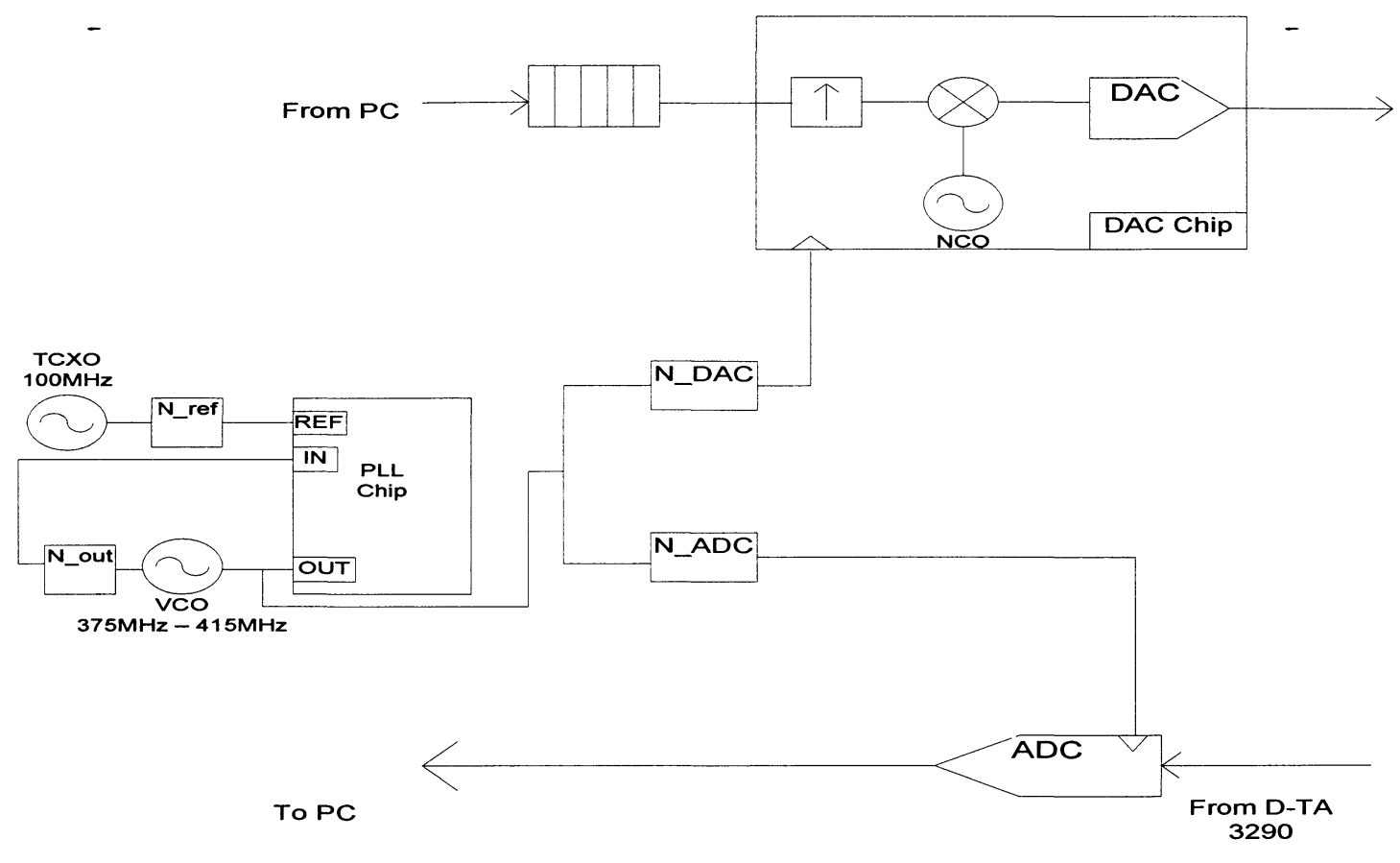

Figure 2.5: Block Diagram for D-TA 2300 


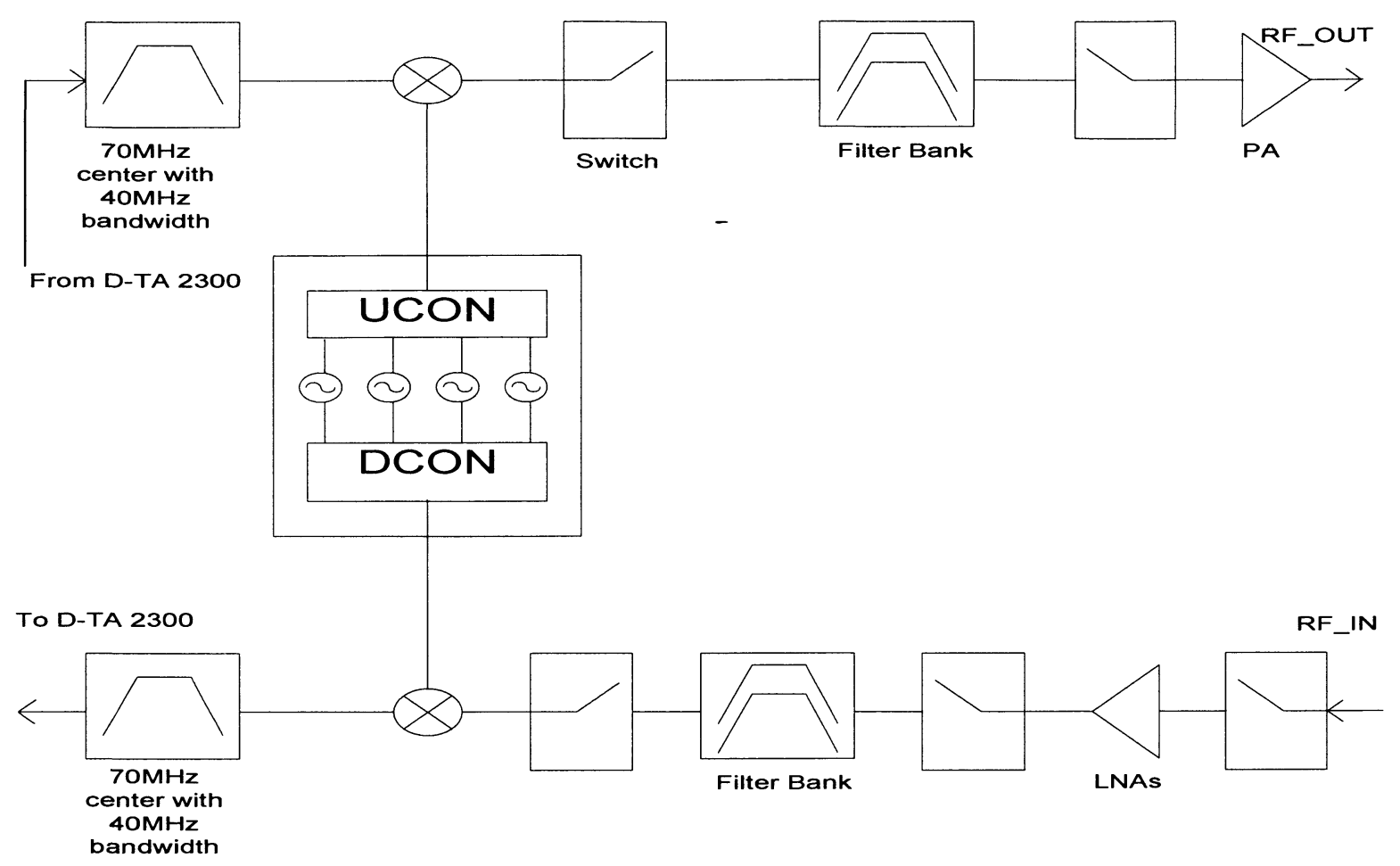

Figure 2.6: Block Diagram for D-TA 3290 (RF Up-converter)

\subsection{Specifications}

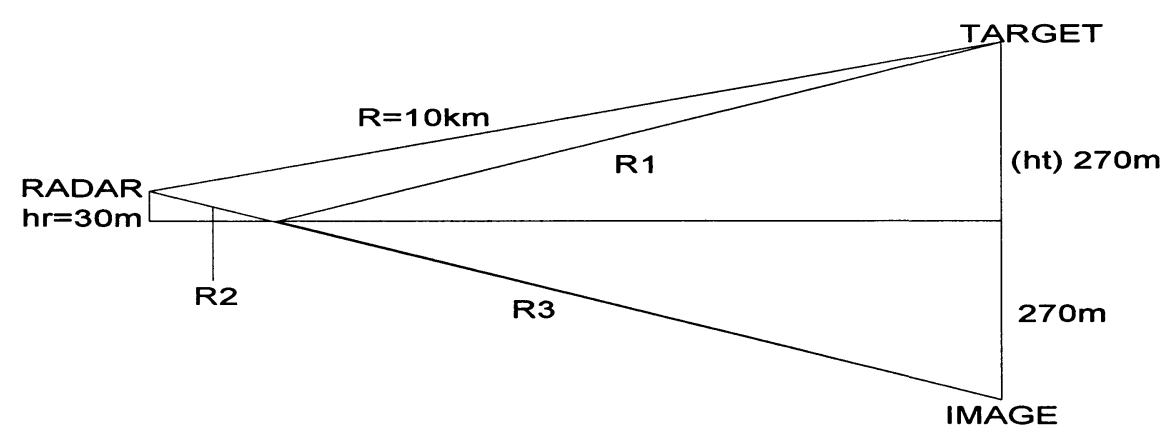

Figure 2.7: Radar Geometry

Figure 2.7 shows the line of sight and multipath echoes of a radar antenna with $30 \mathrm{~m}$ of height (hr) transmitting to a target $10 \mathrm{~km}$ away at a height of $270 \mathrm{~m}(\mathrm{ht})$. The multipath delay is 
derived from the geometry of figure 2.7 . The delay is inversely proportional to range and directly proportional to target height. This implies that the minimum delay is obtained at the maximum range and smallest elevation. The range $(\mathrm{R})$ defines the line of sight echo while R1 and R2 define the interfering echo. With known values of range, elevation, R1 and R2 as depicted in figure 2.7, the expression for multipath delay can be derived as shown below.

$$
\begin{aligned}
& R_{2}+R_{3}=R_{1}+R_{2}=\sqrt{\left(h_{t}+h_{r}\right)^{2}+R^{2}-\left(h_{t}-h_{r}\right)^{2}}=\sqrt{R^{2}+4 h_{t} h_{r}} \\
& \Delta T_{1}=\frac{2 R}{c}, \Delta T_{2}=\frac{\left(R+R_{1}+R_{2}\right)}{c} ;
\end{aligned}
$$

Multipath delay $(\tau)=\Delta T_{2}-\Delta T_{1}=\frac{R_{1}+R_{2}-R}{c}=\frac{\sqrt{R^{2}+4 h_{t} h_{r}}-R}{c}=\frac{R\left(1+\frac{4 h_{t} h r}{R^{2}}\right)^{0.5}-R}{c}=5.4 n s$

Using binomial expansion for $4 h_{t} h_{r} \ll R^{2}$

$\tau \approx \frac{R\left(1+\frac{2 h_{t} h_{r}}{R^{2}}\right)-R}{c}=\frac{2 h_{t} h_{r}}{R * c}$

By setting the target range in figure 2.7 to equal the unambiguous range,

$P R I=\frac{2 R}{c}=2 * \frac{10 k}{3 * 10^{8}}=6.67 * 10^{-5} s=>P R F=15 \mathrm{kHz}$.

The DTA-2300 has a maximum packet size of 4096 samples for the ADC. This implies that the maximum PRI length that can be contained in a single packet is 65.536us, or a minimum PRF of $15.26 \mathrm{kHz}$. The corresponding unambiguous range is $9.8304 \mathrm{~km}$. The maximum range for a single pulse is limited by the D-TA 3290 parameters and is calculated with equation 2.10 . 
Where $P_{t}=10 \mathrm{~W}, G=20 \mathrm{~dB}, \lambda=0.15 \mathrm{~m}, \sigma=1 \mathrm{~m}^{2}, k T_{o}=-174 \mathrm{dBm} / \mathrm{Hz}$, Number of pulses $-\mathrm{n}=1$, Integration Efficiency $-E_{i}(n)=1$, Noise Bandwidth $-B_{n}=40 \mathrm{MHz}$, Noise Factor $-F_{n}=10$, Single pulse SNR $-\left(\frac{S}{N}\right)_{1}=6.02 d B$. Substituting the above D-TA 3290 specifications into the range equation yields a maximum range of $649.54 \mathrm{~m}$. The above equation however, ignores the compression gain $\left(G_{c}\right)$. Including the compression gain in the range equation (2.10) yields the following result in equation 2.14.

$R_{\max }^{4}=\frac{P_{t} G^{2} \lambda^{2} \sigma n E_{i}(n) G_{c}}{(4 \pi)^{3} k T_{o} B_{n} F_{n}\left(\frac{S}{N}\right)_{1}}$

$G_{c}$ in a phase-coded compression scheme is a function of unambiguous range.

$G_{c}=\frac{\Delta t}{T_{c}}=\frac{2 R_{\text {unambig }}}{c T_{c}}$

Where Tc is the chip width and $R_{\text {unambig }}=9.8304 \mathrm{~km}$ from $\mathrm{ADC}$ packet size limitations. Substituting for $G_{c}$ in the range equation yields equation 2.16 .

$R_{\max }^{4}=\frac{2 * P_{t} G^{2} \lambda^{2} \sigma n E_{i}(n) R_{u n a m b i g}}{(4 \pi)^{3} k T_{o} B_{n} F_{n}\left(\frac{S}{N}\right)_{1} c T_{C}}$

The optimum Tc occurs when the signal bandwidth equals the noise bandwidth. To achieve this, $T_{c_{-} o p t}=\frac{2}{B_{n}}=\frac{2}{40 M}=50 \mathrm{~ns}$. Tc is also limited by the baseband sampling rate (Fs) which is $62.5 \mathrm{MHz}$ in this case. Since Tc must be a factor of $1 / \mathrm{Fs}$, The closest $\mathrm{Tc}$ to the optimum is 64ns. Substituting the maximum achievable chip length in addition to the other system parameters into the range equation yields a new maximum range of $3.69 \mathrm{~km}$. To achieve the required unambiguous range, either pulse integration or an increase of transmitted power is 
required. To ensure low power utilization, pulse integration is utilized and at least 80 pulses are required to achieve the unambiguous range.

\subsection{Chapter Summary}

This Chapter shows the derivation of the Radar equation. Based on the Radar equation, curves are derived which show the relationship between the SNR, probability of false alarm and probability of detection. It also shows the probability distribution for classification of Swerling targets. This chapter then provides an overview of the hardware used to implement the waveform testing. Finally, starting parameters for the waveform design are obtained from given specifications and hardware constraints. 


\section{Chapter 3}

\section{Waveform Design}

\subsection{Phase-Coded Pulse Compression}

This thesis is based on the implementation of a system with low peak output power (tens of Watts) which achieves comparable maximum range to systems with kilo-Watts of power. Systems with long pulse widths require less transmitter power but suffer from poor range resolution if pulse compression is not utilized. This implies that a transmitted signal with pulse width equal to the PRI can be optimized for minimal output power provided an optimum pulse compression scheme is chosen. An optimum compression scheme implies maximum gain, minimum side lobes and optimum resolution. The two most common compression schemes are phase coded waveforms and frequency modulated waveforms.

Phase coded waveforms consist of a group of chips/bits where the chip duration is the inverse of the IF bandwidth. Multiplying the chip sequence by the carrier waveform either maintains the phase of the carrier pulses per bit duration or flips the phase by 180 degrees. The received signal delay is obtained by varying the transmitted signal delay, and at each delay the received sequence is multiplied and added with its corresponding transmitted sequence. This process is called auto-correlation. Cross-correlation is realized when the received signal is multiplied and added to a different transmitted sequence. Maximal-length sequences (MLS) have a correlation peak value equal to the number of chips and a value of -1 at every other delay. These sequences are derived from primitive polynomials and have 
randomness characteristics similar to those ascribed to truly random sequences. Any polynomial can be implemented in hardware with shift registers and an X-OR gate. The number of shift register stages (n) determine the length of the sequence and highest degree of the polynomial while the inputs to each X-OR gate determine the type of polynomial. If the generated polynomial is primitive, a maximal-length sequence is obtained with a length of $N=2^{n}-1$ before repetition. Given $\mathrm{n}$ shift register stages, the total number of maximal length sequences is given by equation 3.0.

$M=\frac{N}{n} \Pi\left(1-\frac{1}{p_{i}}\right)$

Where $p_{i}$ represents the prime factors of $\mathrm{N}$ [20]. Since a maximal length sequence is repetitive after $\mathrm{N}$ chips, PRIs with pulse widths equal to $N T_{\text {chip }}$ achieve pulse compression but still suffer from range ambiguity. Range ambiguity can be resolved by transmitting a different sequence every PRI. In this way, unambiguous targets are processed using the autocorrelation function while ambiguous targets are processed using cross-correlation. A transmission scheme implemented in this manner with a different MLS per PRI is hardware intensive because $\mathrm{M}$ primitive polynomials for a specific number of shift registers (n) have to be found and a different polynomial implemented per PRI. An alternative to this is using truncated portions of a single MLS for each PRI with the drawback being higher side-lobe levels for certain sequences as shown in figure 3.1. 


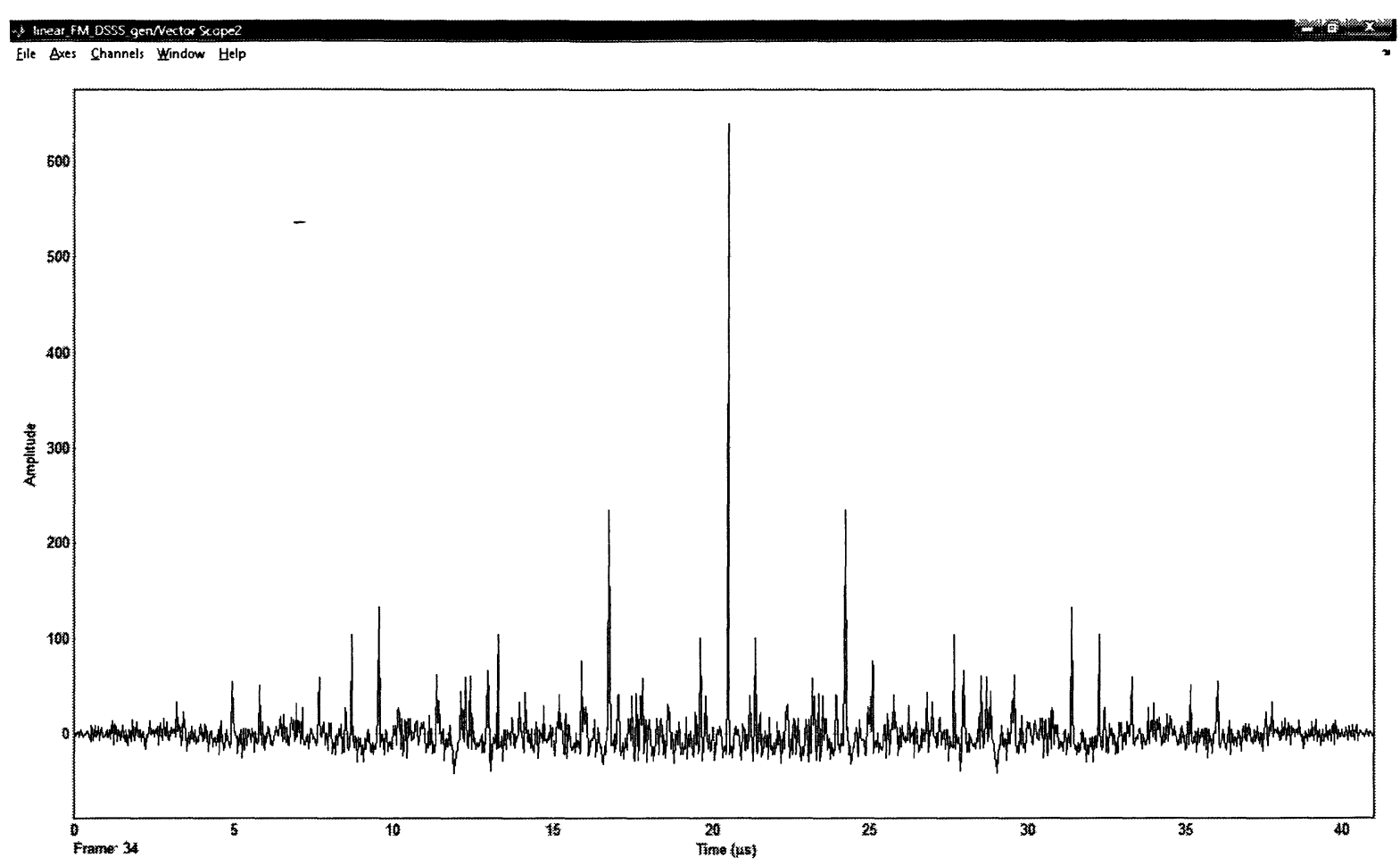

Figure 3.1: Auto-Correlation of MLS with $n=20$ and $N=1,048,575$ truncated to 640 bits per PRI

\subsection{Frequency Modulation Compression}

Frequency modulation is another form of pulse compression and it operates by changing the carrier frequency over a specified bandwidth and time. Frequency modulated signals are of the form: $y(t)=A \sin \left(2 \pi f_{c} t+\theta(t)\right)$, where $\theta(t)$ varies according to the type of modulation. Since the desired waveform property as a result of frequency modulation is high resolution; the best type of frequency modulation to use is linear FM because its autocorrelation response has the narrowest main lobe. For a linear FM signal, $\quad \theta(t)=\beta t^{2}$ where $\beta$ is the frequency slope. The difference-frequency resulting from the product of the 
sent and received signals has a constant frequency offset $(\Delta \mathrm{f})$ which depends on the target range [7]. If $\Delta \mathrm{f}$ is known, target delay $\left(T_{r}\right)$ can be obtained from equation 3.1.

$T_{r}=\frac{\Delta f}{\Delta F} * \tau=\frac{2 R}{c}$

Where $\tau$ is the chirp period, $\Delta F$ is the difference between the maximum and minimum frequency, $R$ the range and $c$ the speed of light. This implies that theoretically, frequency modulated waveforms can have no resolution limit. However, in digital systems, the smallest observable delay and hence resolution limit is the inverse sampling frequency. For digital reception and processing, range and Doppler from linear FM pulses can be obtained by correlation and filtering respectively in that order. The processing is identical to that for phase-coded waveforms except that phase-codes use linear correlation while linear FM uses circular correlation. Analytical derivation of the linear FM auto-correlation function is shown below.

$y_{\text {chirp }}=\mathrm{e}^{\mathrm{j}\left(w n+\beta n^{2}\right)}$, w is the starting angular frequency, $\beta$ the angular frequency slope and $n$ the discrete time.

$A_{c}(n)=\sum_{k=-\infty}^{k=\infty} \mathrm{e}^{\mathrm{j}\left(w k+\beta k^{2}\right)} \mathrm{e}^{\mathrm{j}\left(w(k+n)+\beta(k+n)^{2}\right)}$, where $A_{c}(n)$ represents circular autocorrelation. From $\mathrm{Z}$ transform properties, If $h(n)=\mathrm{e}^{\mathrm{j}\left(w n+\beta n^{2}\right)}$

Then $A_{F}(f)=H(f) \cdot(H(f))^{*}$

Where $A_{F}(f)=F\left\{A_{c}(n)\right\} \& H(f)=F\{h(n)\}$ 


$$
\begin{aligned}
& H(f)=\sqrt{\frac{\pi}{-j \beta}} e^{-\frac{\pi f^{2}}{j \beta}} \text { from Fourier transform properties implying } \\
& A_{F}(f)=\sqrt{\frac{\pi}{-j \beta}} e^{-\frac{\pi\left(f-\frac{w}{2 \pi}\right)^{2}}{j \beta}} * \sqrt{\frac{\pi}{j \beta}} e^{\frac{\pi\left(f-\frac{w}{2 \pi}\right)^{2}}{j \beta}}=\frac{\pi}{\beta} \\
& =>A_{c}(n)=F^{-1}\left\{A_{F}(f)\right\}=\frac{\pi}{\beta} \delta(n) .
\end{aligned}
$$

Note that the above Fourier transforms are valid for an infinite time domain signal. From the above derivation, it can be observed that the auto-correlation of an infinite time and continuous linear chirp yields a delta function scaled by a constant. For practical purposes, an infinite time domain signal is unachievable and as a result, the auto-correlation deviates from the ideal delta function by introducing side-lobes and degrading peak resolution. The non idealities can be observed in figure 3.2 which is the auto-correlation response of a linear chirp ranging from 0 to $15.625 \mathrm{MHz}$ with a $62.5 \mathrm{MHz}$ sampling frequency (16ns sampling period). Based on the range and sampling frequency, $\beta=2 * p i * \frac{15.625 e 6}{1023 * 1.6 e-8}=$ $\frac{5.998 E 12 \mathrm{rad}}{\mathrm{s}^{2}}, N=1023.1023$ values of $\mathrm{h}(\mathrm{n})$ are obtained from $0 \mathrm{~s}-1023 * 1.6 \mathrm{E}-8 \mathrm{~s}$. The autocorrelation shown in figure 3.2 is obtained by finding the Fourier transform of $h(n)$, multiplying by its conjugate and taking the inverse Fourier transform. 


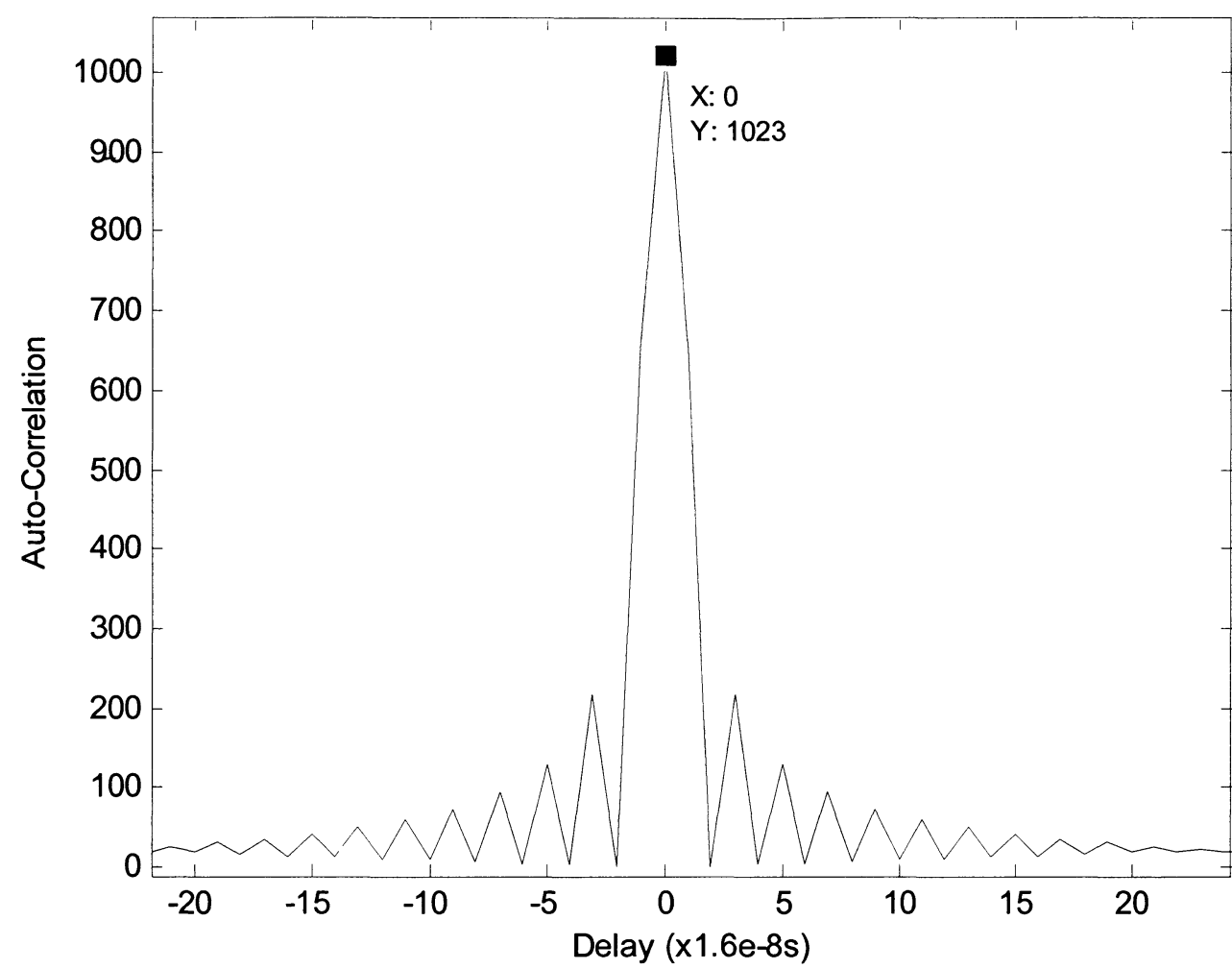

Figure 3.2: Auto-Correlation of linear-FM chirp

It can also be observed from the auto-correlation function derivation that the starting frequency for the chirp given by ' $w$ ' does not affect the auto-correlation response hence the transmitted signal can be simplified to $h(n)=\mathrm{e}^{\mathrm{j}\left(\beta n^{2}\right)}$.

\subsection{Phase-Coded-Linear-FM}

Linear FM pulses have superior resolution over phase-coded compression because the chip width limitation of the phase coded waveform is IF bandwidth whereas a frequency modulated waveform can change at the sampling frequency. Since the processing of both 
linear FM and phase-coded waveforms is similar, a compression scheme can be designed which exploits the advantages of both waveforms. Convolving a triangular FM pulse with a random pulse train yields such a compression scheme. Convolution ensures that the delta function auto-correlation responses of both the phase-codes and linear chirps are maintained in the combined waveform. The random pulse train ensures that returns outside the transmitted pulse train are cross-correlated and this brings about rejection of ambiguous targets. The derived waveform benefits from improved resolution as target resolution is determined by the triangular FM pulse. It also benefits from a lack of range ambiguity as a result of phase coding because random pulse correlation ensures that second time around (and higher) targets are rejected. In order to reject ambiguous targets, echo processing should be a cross-correlation as opposed to auto-correlation for unambiguous targets. Since the linear FM signal is repetitive every PRI, its cross-correlation is equivalent to its autocorrelation. Phase codes on the other hand have separate auto-correlation and crosscorrelation properties. The combination of phase codes and linear FM properties in a single waveform should be done in such a way that the delta function auto-correlation property is preserved. Preservation of the delta function auto-correlation property is done by convolution of a phase-coded waveform with a linear FM waveform and analytical proof is shown below.

$$
\begin{aligned}
& h(n)=\mathrm{e}^{\mathrm{j}\left(\beta n^{2}\right)} \otimes \mathrm{e}^{\mathrm{j}(\theta(n))} \\
& A_{c}(n)=\sum_{k=1}^{k=N}\left(\mathrm{e}^{\mathrm{j}\left(\beta k^{2}\right)} \otimes \mathrm{e}^{\mathrm{j}(\theta(k))}\right)\left(\mathrm{e}^{\mathrm{j}\left(\beta(k+n)^{2}\right)} \otimes \mathrm{e}^{\mathrm{j}(\theta(k+n))}\right) \\
& =>A_{F}(f)=F\left\{\mathrm{e}^{\mathrm{j}\left(\beta n^{2}\right)} \otimes \mathrm{e}^{\mathrm{j}(\theta(n))}\right\} * F\left\{\mathrm{e}^{-\mathrm{j}\left(\beta n^{2}\right)} \otimes \mathrm{e}^{-\mathrm{j}(\theta(n))}\right\}=\frac{\pi}{\beta} * N
\end{aligned}
$$


A convolution in time domain is equivalent to a multiplication in the frequency domain thus the above expression reduces to the multiplication of the Fourier transform of each of the individual components. The linear FM products reduce to $\frac{\pi}{\beta}$ while the phase coded products reduce to the number of chips $(\mathrm{N})$.

$$
=>A_{c}(n)=\frac{\pi}{\beta} * N * \delta(n)
$$

From the auto-correlation expression above, we can notice that the correlation gain of the chirp has been multiplied by the correlation gain of the phase coded waveform. Figure 3.3 shows the auto-correlation response of the convolution of the linear FM waveform with a 1023 chip maximal length sequence. Since the phase modulated signal is a MLS, the convolved waveform shown in figure 3.3 is a approximately equivalent to the product of the response shown in figure 3.2 and a discrete ideal delta function. The correlation gain can be observed to be $1.046 \mathrm{E} 6$ as determined by the product of the chirp gain (1023) and the number of MLS chips (1023). In reality, the extra factor of $\mathrm{N}$ in the correlation gain of the phase-coded-linear-FM signal is not achievable for a fixed output power level because the power of the convolved waveform is the sum of the chirp power and phase coded waveform power in $\mathrm{dB}$. The auto-correlation expression above also shows us that the applying a phase coded waveform to the chirp does not affect its correlation response thus the radar system can benefit from the high resolution of a chirp compared to a phase-coded waveform for a fixed bandwidth. The cross correlation derivation of the phase-coded-linear-FM waveform is shown below. 
$X_{c}(n)=\left(\mathrm{e}^{\mathrm{j}\left(\beta n^{2}\right)} \otimes \mathrm{e}^{\mathrm{j}(\theta(n))}\right) \otimes\left(\mathrm{e}^{-\mathrm{j}\left(\beta n^{2}\right)} \otimes \mathrm{e}^{-\mathrm{j}(\alpha(n))}\right)$. From the associative convolution property, this can be rearranged to:

$$
\begin{aligned}
& X_{c}(n)=\left(\mathrm{e}^{\mathrm{j}\left(\beta n^{2}\right)} \otimes \mathrm{e}^{-\mathrm{j}\left(\beta n^{2}\right)}\right) \otimes\left(\mathrm{e}^{\mathrm{j}(\theta(n))} \otimes \mathrm{e}^{-\mathrm{j}(\alpha(n))}\right)=\frac{\pi}{\beta} \delta(n) \otimes\left(\mathrm{e}^{\mathrm{j}(\theta(n))} \otimes \mathrm{e}^{-\mathrm{j}(\alpha(n))}\right) \\
& =>X_{c}(n)=\frac{\pi}{\beta}\left(\mathrm{e}^{\mathrm{j}(\theta(n))} \otimes \mathrm{e}^{-\mathrm{j}(\alpha(n))}\right)
\end{aligned}
$$

Where $\alpha(n)$ and $\theta(n)$ are two different PN sequences. Optimally, the cross-correlation should be 0 but PN sequences have poor cross-correlation properties with cross-correlation values of up to $0.35 * \mathrm{~N}$ for certain sequences [8]. Due to the relatively small number of maximal length sequences for a given $\mathrm{N}$, partial sequences have to be used for correlation.

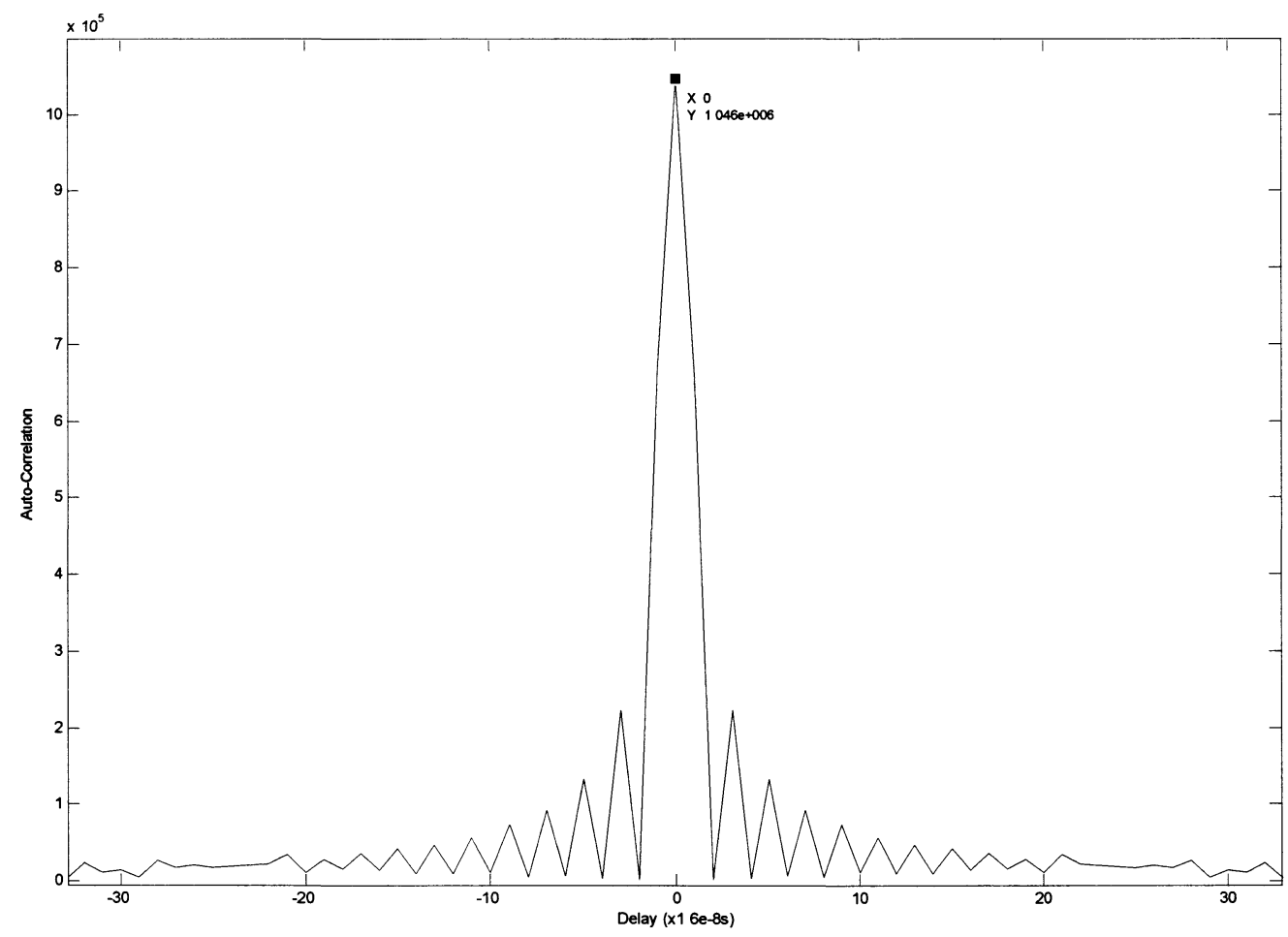

Figure 3.3: Auto-Correlation of convolved phase modulated and linear-FM signal 27 
The drawback to using partial length sequences (PLS) is their auto-correlation response approximates the delta function. The auto-correlation gain of a PLS is equal to the length of the sequence as in a MLS but the PLS contains side-lobes as opposed to no side-lobes from a MLS [9]. Given the following base-bandwidth restriction: $B=\frac{1}{T_{c}}=\frac{1}{4 T_{s}}=\frac{1}{4 * 1.6 E-8}=$ 15.625 M Hz, and PRI restriction of 65.55us, the chirp gain (B*PRI) is 1024 and the number of phase-code chips is 1024. Figure 3.4 shows the cross-correlation or second time around target rejection over multiple partial sequences of the transmitted waveform. The transmitted waveform is obtained by the circular convolution of a chirp with bandwidth-time product of 1024 and a truncated PN sequence consisting of 1024 chips from a 20-bit LFSR. It can be observed that the attenuation factor ranges from 0 to 0.25 with an average of 0.15 .

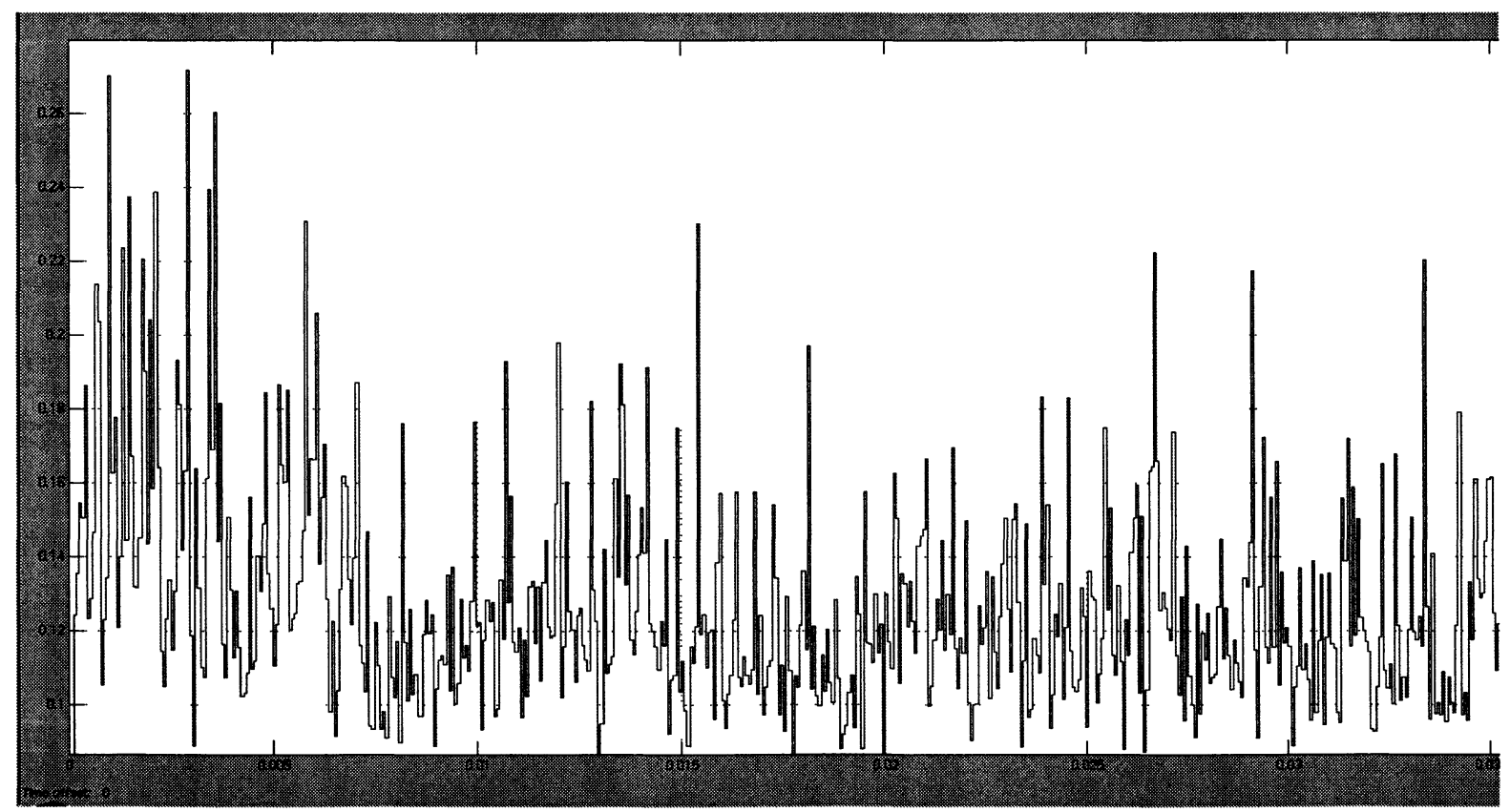

Figure 3.4: Normalized peak cross-correlation value over successive pulses 


\subsection{Chapter Summary}

This chapter examines the properties of both phase and frequency modulated waveforms and derives their individual auto-correlation responses. It then uses their individuatcorrelation responses to derive the auto-correlation response of the phase-coded-linear-FM signal.

Finally, this chapter looks at the cross-correlation properties of the phase-coded-linear-FM signal. 


\section{Chapter 4}

\section{Radar Simulation in Matlab}

\subsection{Transmitter}

This chapter generates and simulates a model in Simulink to verify the ability of the proposed waveform to reject noise and jamming, accurately detect targets in the presence of multipath, and detect range and Doppler in the presence of noise.

As derived from the radar specifications, a chip rate (inverse of chip length $-T_{c}$ ) of $15.625 \mathrm{MHz}$ at a $62.5 \mathrm{MHz}$ sampling rate is required to achieve maximum compression gain. The PRI is determined in the specifications to be 65.536 us. The phase-coded-linear-FM waveform also compresses the pulse to achieve finer resolution given by $R=\frac{c \Delta t}{2}=$ $\frac{3 E 8 * 1.6 e-8}{2}=2.4 m$, where $\Delta t$ is the inverse of the sampling frequency. Figure 4.1 shows the Simulink block diagram used to implement the phase-coded-linear-FM waveform. The phase-codes or chips are obtained from a 20 bit LFSR and converted to a bipolar from a

unipolar format. The number of chips per PRI is obtained as follows: $N=\frac{P R I}{T_{c}}=1024$. These chips are convolved with a triangular FM pulse ranging from DC to $15.625 \mathrm{MHz}$ and lasting 65.536us. Note that the FM compression gain given by its bandwidth-time product is equal to the number of phase coding chips. Figures 4.2 and 4.3 show the spectra of both chirp and phase modulated waveforms respectively; the product of one spectrum with the 
conjugate of the other yields the spectrum of the phase-coded-linear-FM waveform in figure 4.4.

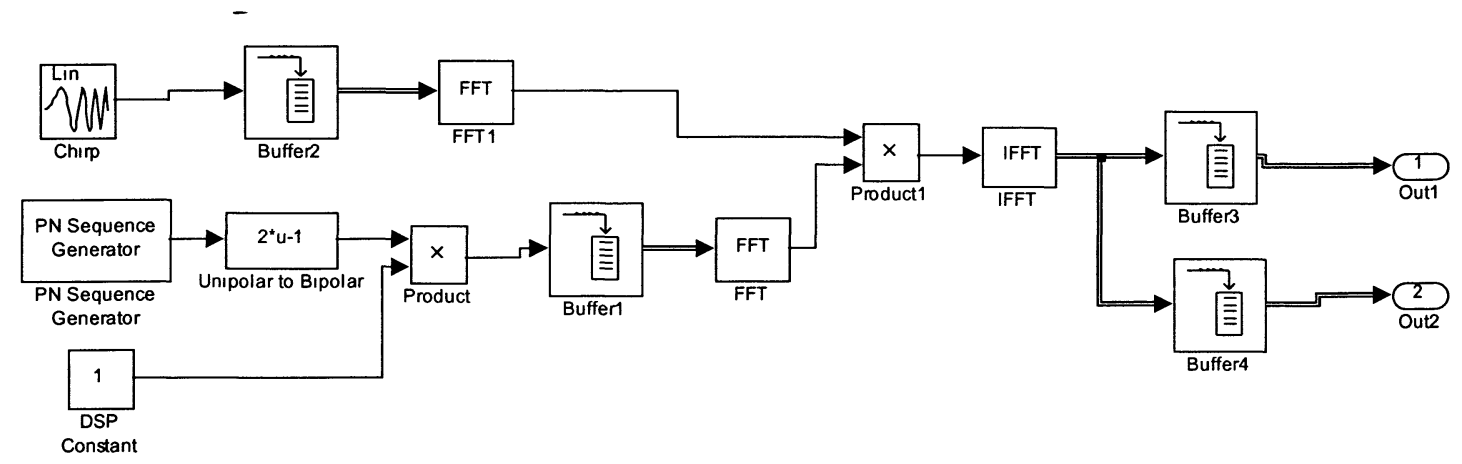

Figure 4.1: Block Diagram for the transmitter

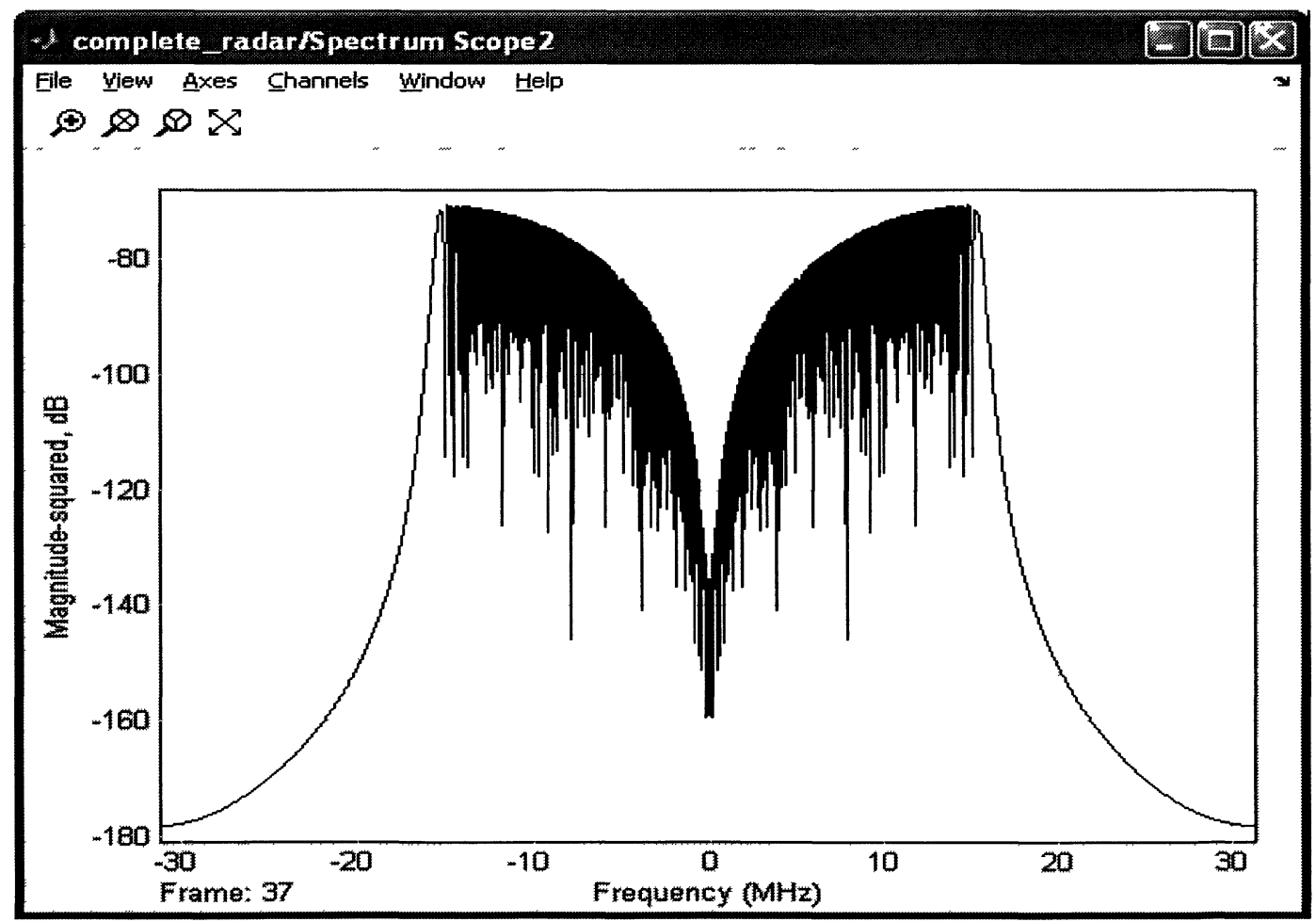

Figure 4.2: Spectrum of triangular FM chirp 


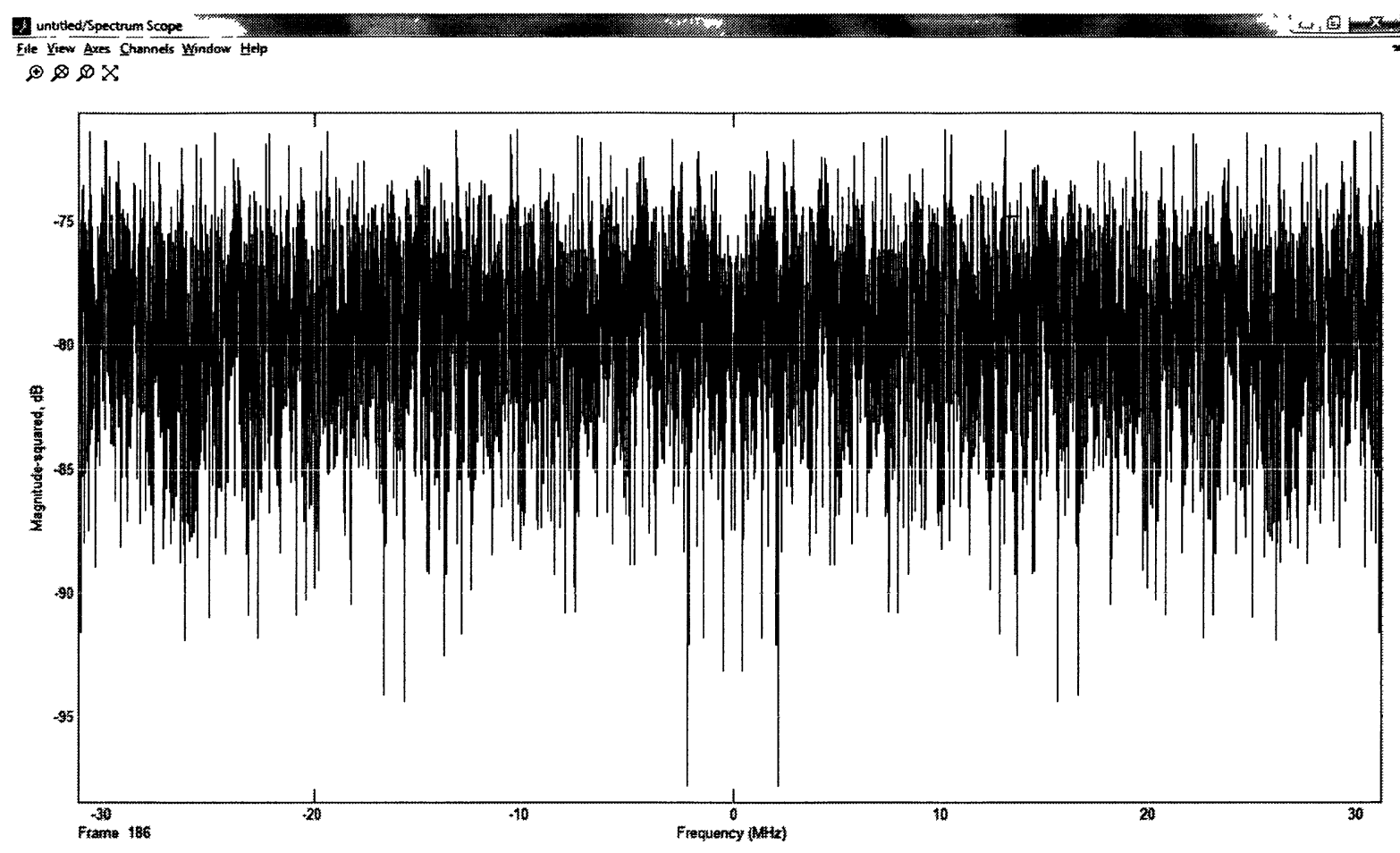

Figure 4.3: Spectrum of phase modulated signal

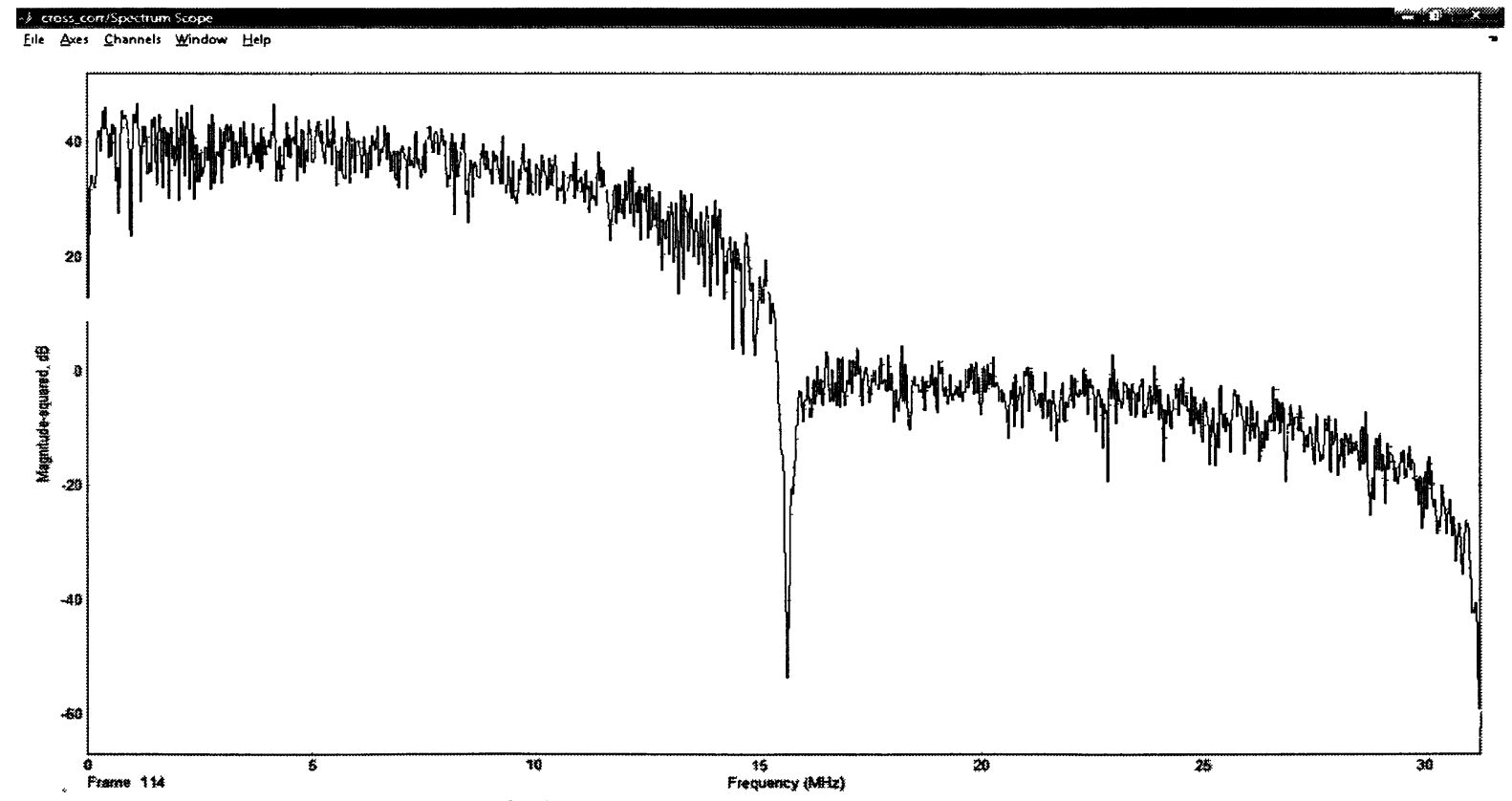

Figure 4.4: Spectrum of phase-coded-linear-frequency-modulated signal 
The spectrum shown in figure 4.2 is that of a triangular FM signal and it is different from the spectrum of a linear FM signal which is approximately flat. The equal but opposite frequency slopes of the triangular FM signal cause the resulting null at DC. The phase modulated signal is pseudo-random noise hence its approximately flat spectrum as shown in figure 4.3.

\subsection{Channel}

The channel effects considered in this model are multipath, noise, target cross-section and Doppler shift. As shown in the Specification section, multipath delay depends on the range and elevation of a particular target. Individual multipath components cannot be separated unless the multipath delay is greater than the time equivalent of the target resolution as determined by the transmitted waveform. In this case, target resolution is determined by the inverse of the sampling frequency $-16 \mathrm{~ns}$. For range measurements, the ability of the receiver to separate multipath components does not affect range accuracy as long as the path with minimum delay is chosen. As a result degradation in accuracy due to multipath only occurs when determining target elevation. Multipath is modeled by adding a delayed version of the transmitted signal to the transmitted signal. Since the LOS and delayed paths are different, their respective free space losses and antenna gains are computed before addition. Additive White Gaussian noise is generated by an existing Simulink block and added to the transmitted signal with a signal to noise ratio at the receiver of $10 \mathrm{~dB}$ including processing gain and $-20 \mathrm{~dB}$ without processing gain. Free space path loss is computed by the expression in equation 4.0. 
$L=\frac{\lambda^{2}}{(4 \pi)^{2} R^{4}}$

Implying an amplitude attenuation of $\sqrt{L}$. Target Doppler is modeled by applying a frequency shift to the carrier given by equation 4.1

$f_{d}=\frac{v f_{c}}{c}$

Where $v$ is target velocity, $f_{c}$ is the carrier frequency and $c$ the speed of light. The target constitutes part of the channel and is implemented as a delay in echo reception. Moving targets have variable delays in accordance to their velocities. Clutter also constitutes part of the radar channel and it comprises of stationery and sea clutter. Stationery clutter results from non-moving targets; as a result their echoes have low Doppler shifts though not quite zero as a result of antenna rotation. The sea comprises of waves which are due to the earth's rotation and wind velocity, as a result echoes returning from sea waves have a non-zero Doppler shift. Empirical data suggests that the clutter spectrum has a Gaussian shape with an additional peak at the edge of the spectrum due to the main beam of the antenna [10]. Only the main beam clutter frequency as determined by the sea state and wind velocity is implemented in this model. The virtual sea clutter velocity at the peak of the clutter spectrum for $\mathrm{X}$ and $\mathrm{C}$ band radar looking upwind at low grazing angles can be estimated by equation 4.2.

$V_{v i r} \approx 0.25+0.13 U$

Where $U$ is the wind speed [11]. Wind speeds vary from $0 \mathrm{~m} / \mathrm{s}$ for calm seas to $36.9 \mathrm{~m} / \mathrm{s}$ for hurricanes [12]. This implies that virtual clutter velocities range from approximately $0.25 \mathrm{~m} / \mathrm{s}$ to $5.047 \mathrm{~m} / \mathrm{s}$ under various sea conditions. The resulting Doppler frequencies are $3.3 \mathrm{~Hz}$ to $67.3 \mathrm{~Hz}$ at a carrier frequency of $4 \mathrm{GHz}$. The Simulink Channel model is shown in figure 4.5. 
Clutter returns (as shown in figure 4.5 as delays $15-18$ ) are positioned before the target range bin (delay 21 in figure 4.5) to ensure the receiver's ability to detect a target in the presence of clutter. The delay number represents the distance from the target to the Radar. The magnitude of the auto-correlation response depends on the target cross-section and the transmitted phase-code sequence. Clutter positioning in figure 4.5 can also be used to verify the receiver's ability to distinguish the target from delayed (multipath) components of the transmitted signal. More multipath components can be used in figure 4.5 but the purpose of this test is to verify the receiver`s ability to distinguish components separated by more than one delay unit.

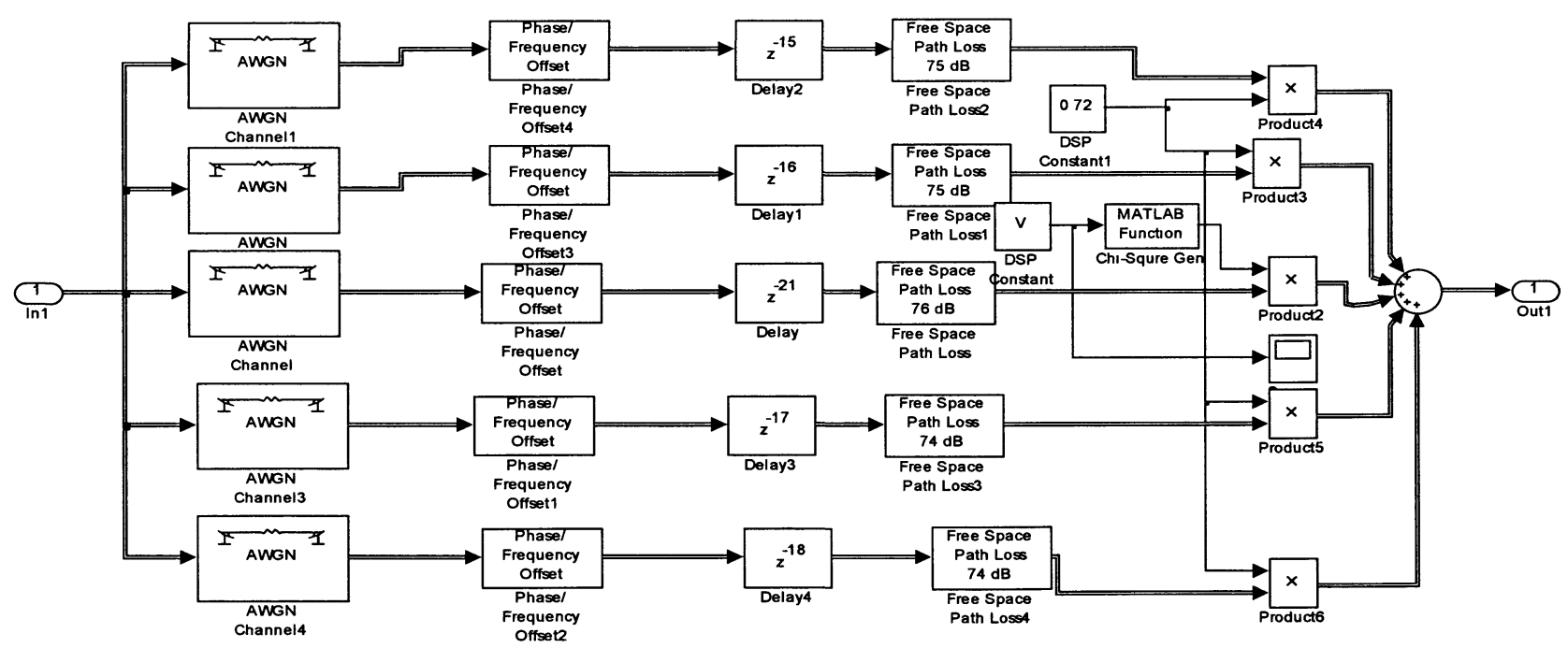

Figure 4.5: Block Diagram for Radar Channel

\subsection{Receiver Processing}

The receiver is implemented by buffering the received bits per PRI and correlating the entire PRI with the transmitted sequence. The correlation processing gain (pulse width/chip length) 
due to the phase coded effect of the waveform is $10 \log (\mathrm{N})=10 \log (1024)=30.1 \mathrm{~dB}$ since 1024 chips are sent per pulse [13]. The correlation processing gain due to the frequency modulation property of the waveform is the bandwidth-time product: $10 \log (\mathrm{BT})=10 \log$ $(15.625 \mathrm{M} * 65.536 \mathrm{u})=30.1 \mathrm{~dB}$. The total processing gain is the sum in $\mathrm{dB}$ of the individual processing gain yielding a total gain of $60.2 \mathrm{~dB}$ used to improve the receiver SNR hence the probability of detection. Since the transmitted waveform is continuous, each received PRI contains both the current and previous transmitted sequence. As a result, correlation is implemented by taking the FFT of the received sequence and the last two transmitted sequences. The received PRI spectrum is multiplied by the complex conjugate of the transmitted sequence spectrum before taking an inverse FFT to yield the time domain autocorrelation response. It is advantageous to use FFTs for correlation of long sequences because the processing time is $3 N * \log N+N$ compared to $N^{2}$ for a traditional correlation algorithm where $\mathrm{N}$ is the number of samples per PRI [14]. The receiver block diagram in figure 4.6 shows the correlation processing. Depending on the target cross-section, the autocorrelation value for the target range bin varies. Figure 4.7 shows the auto-correlation response for the Swerling III and IV pdf while figure 4.8 shows the auto-correlation response for the Swerling I and II pdf. The target range bin is the second peak in both figures 4.7 and 4.8 which appears at 32.94 us. Due to the nature of the Swerling I and II pdf, the cross-section returns are much higher compared to Swerling III and IV as can be observed from target auto-correlation values shown in figure 4.7 and 4.8. The receiver can also distinguish returns separated by at least $16 \mathrm{~ns}$ as shown in figure 4.7 and 4.8 thus verifying the receiver's ability to distinguish LOS from multipath components. Also, since the delay between the clutter and 
target peaks as shown in figure 4.8 and 4.9 is 48 ns. The waveform range resolution is also verified for targets separated by 3 range resolution bins. The maximum peak corresponds to a clutter return with a virtual Doppler frequency of $67 \mathrm{~Hz}$ while the second most prominent peak corresponds to a target moving a velocity of $300 \mathrm{~m} / \mathrm{s}$.

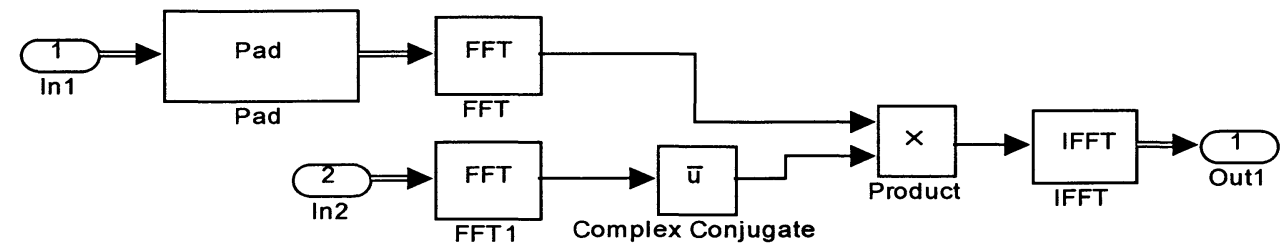

Figure 4.6: Block Diagram for Receiver Correlation Processing

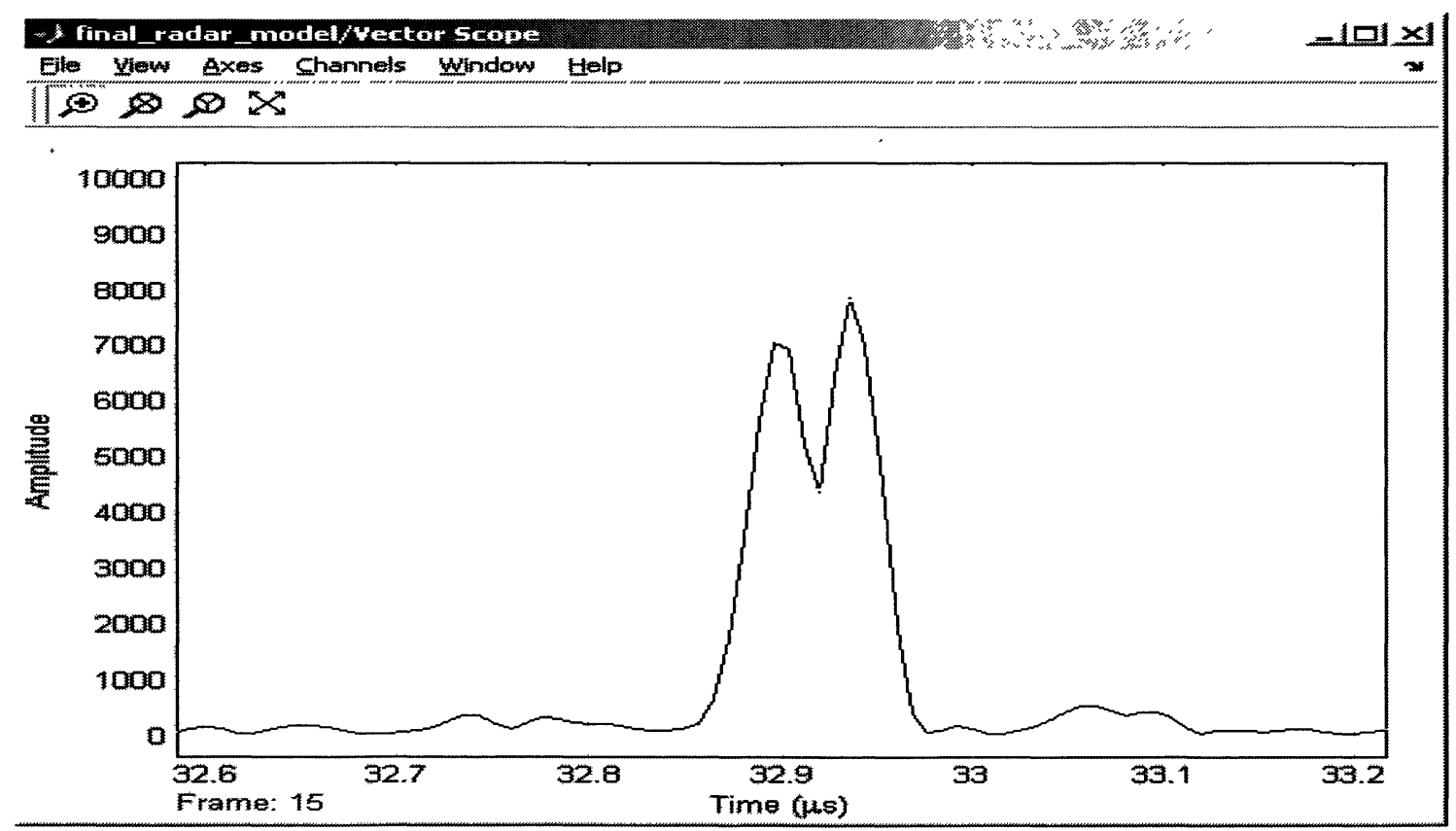

Figure 4.7: Simulated Auto-Correlation Response for a Swerling III or IV target 


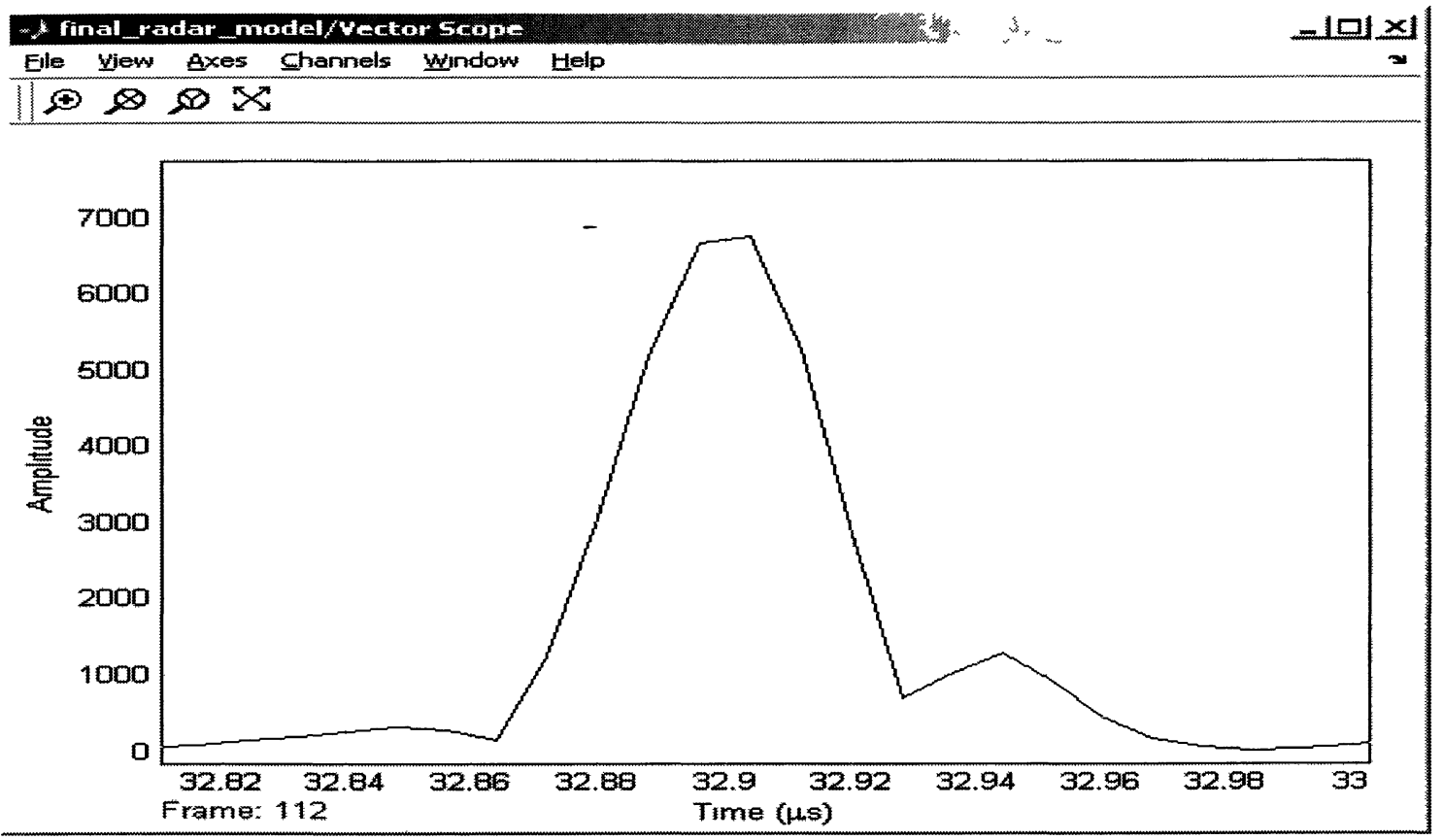

Figure 4.8: Simulated Auto-Correlation Response for a Swerling I or II targets

The Doppler frequency is obtained by computing the FFT of the complex auto-correlation result over successive pulses. Computing the Doppler shift in this way is called Serial Doppler processing. Since the waveform is a Spread Spectrum, the carrier is masked thus correlation should be used to extract the return from the noise floor before processing. Figure 4.9 shows the spectrum of auto-correlation values sampled at the PRF for the target range bin obtained from figure 4.8. The auto-correlation values are stored over 256 pulses for a target moving at $300 \mathrm{~m} / \mathrm{s}$ and a signal with carrier frequency of $4 \mathrm{GHz}$. The required Doppler frequency given the above specifications is $4 \mathrm{kHz}$ (from $f_{d}=\frac{v f_{c}}{c}$ and the frequency which provides the peak magnitude obtained from a 256 point FFT is observed from figure 4.9 to 4.12 to coincide with this value. Figure 4.9 shows the Doppler frequency at the target range 
bin for a Swerling I target. Frequency shifts due to the target and clutter are observable at this range bin because the target cross-section is much smaller than the clutter cross-section. For a Swerling II target, the target Doppler shift is again observed at the expected frequency (as shown in figure 4.11) although, the carrier to noise ratio is less than that for Swerling I targets because of the pulse to pulse variation in cross-section. Swerling III has a high carrier to noise ratio (as shown in figure 4.10) like Swerling I but without the clutter Doppler shift because the Swerling III cross-section is much higher that the clutter cross-section. The carrier to noise ratio for a Swerling IV target is smaller than that of Swerling III as observed in figure 4.12 due to pulse to pulse cross-section variation. Its carrier to noise ratio is also greater than that of Swerling II due to its higher cross-section return and no dominant peak is observed at the clutter Doppler frequency for the same reason. The Doppler frequency at the clutter range bin is shown in figure 4.13 and it shows a peak frequency at $67 \mathrm{~Hz}$. The received clutter has a high carrier to noise ratio as clutter is simulated with a non-fluctuating crosssection. 


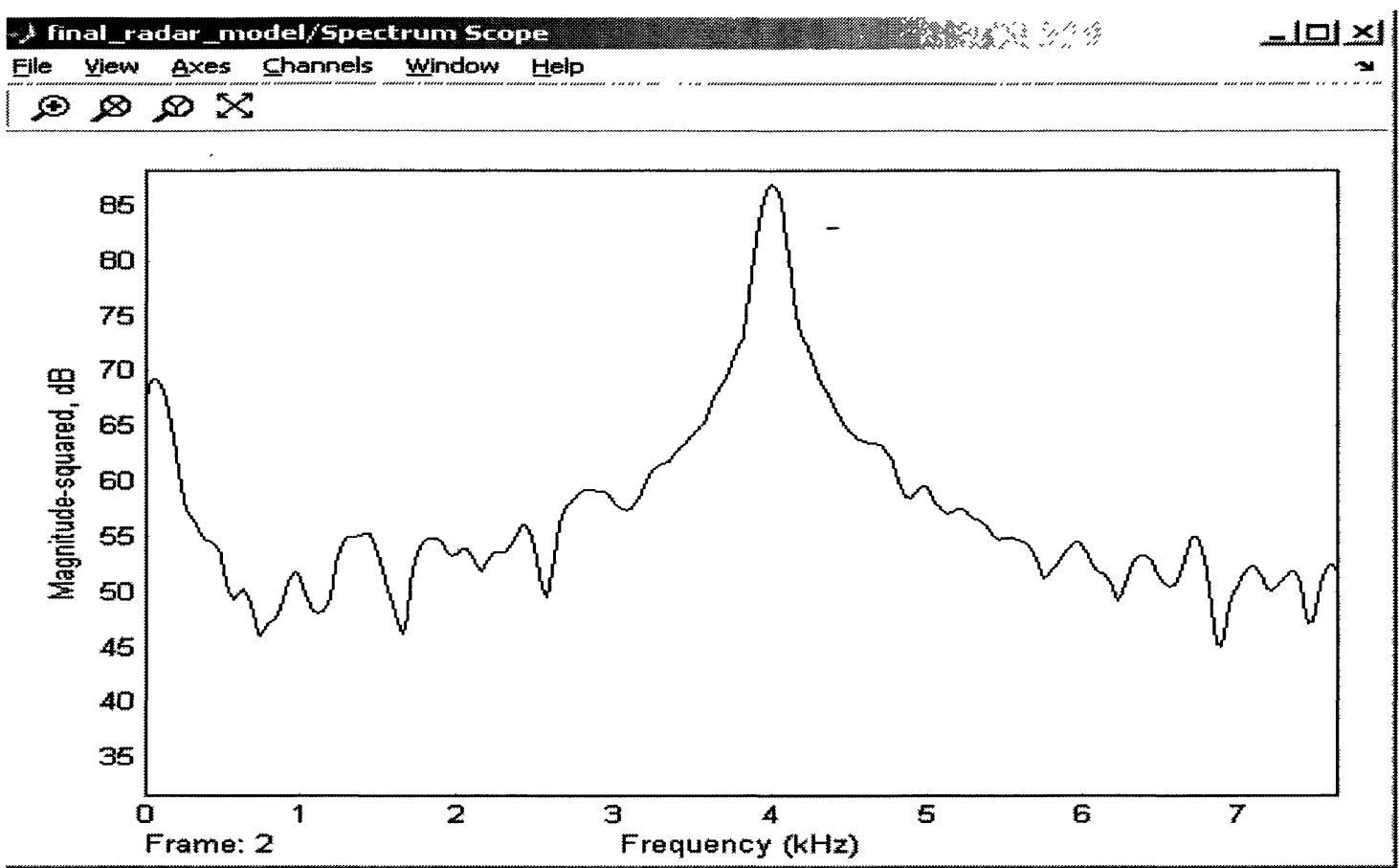

Figure 4.9: Doppler Spectrum for Swerling I target
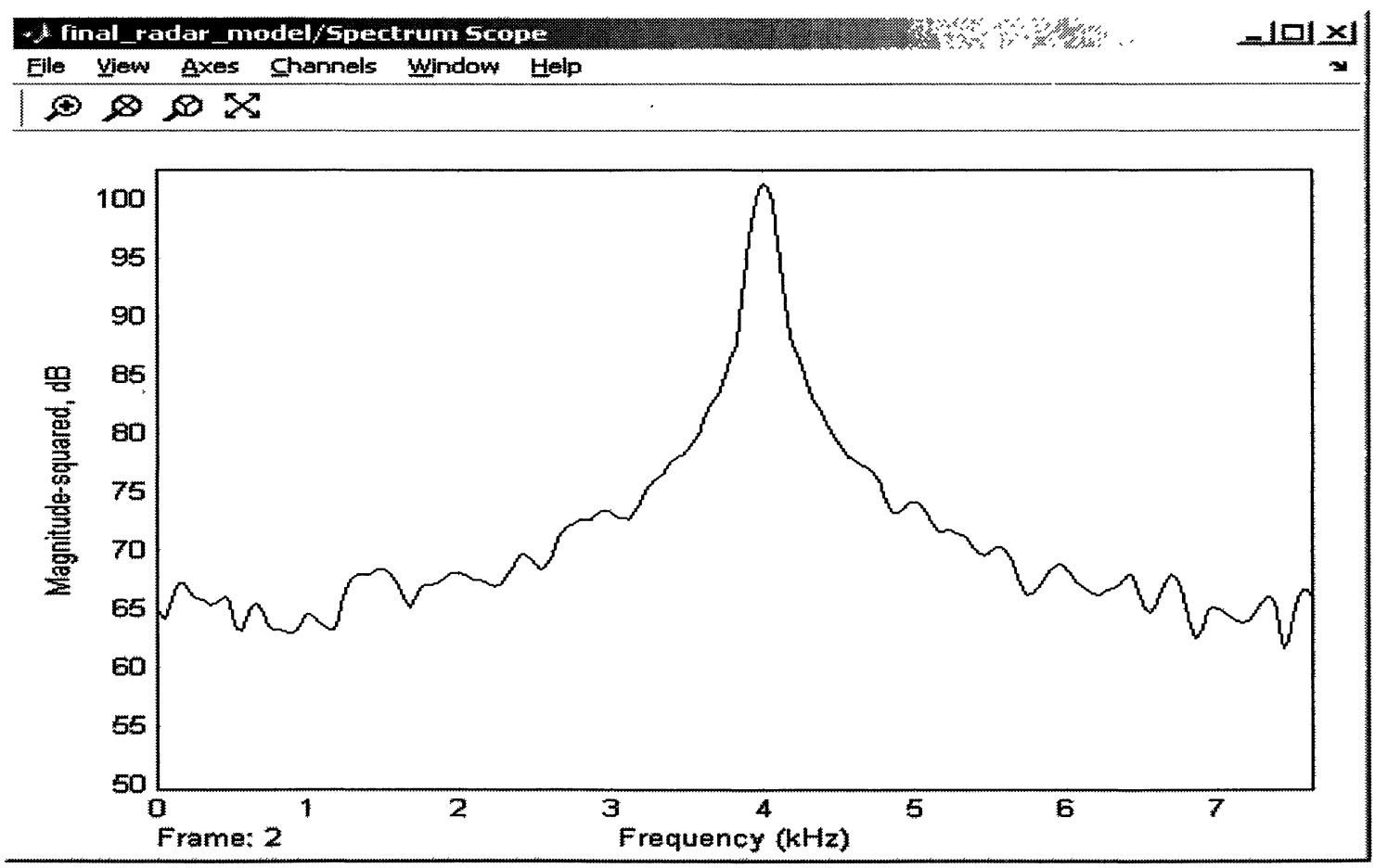

Figure 4.10: Doppler Spectrum for Swerling III target 


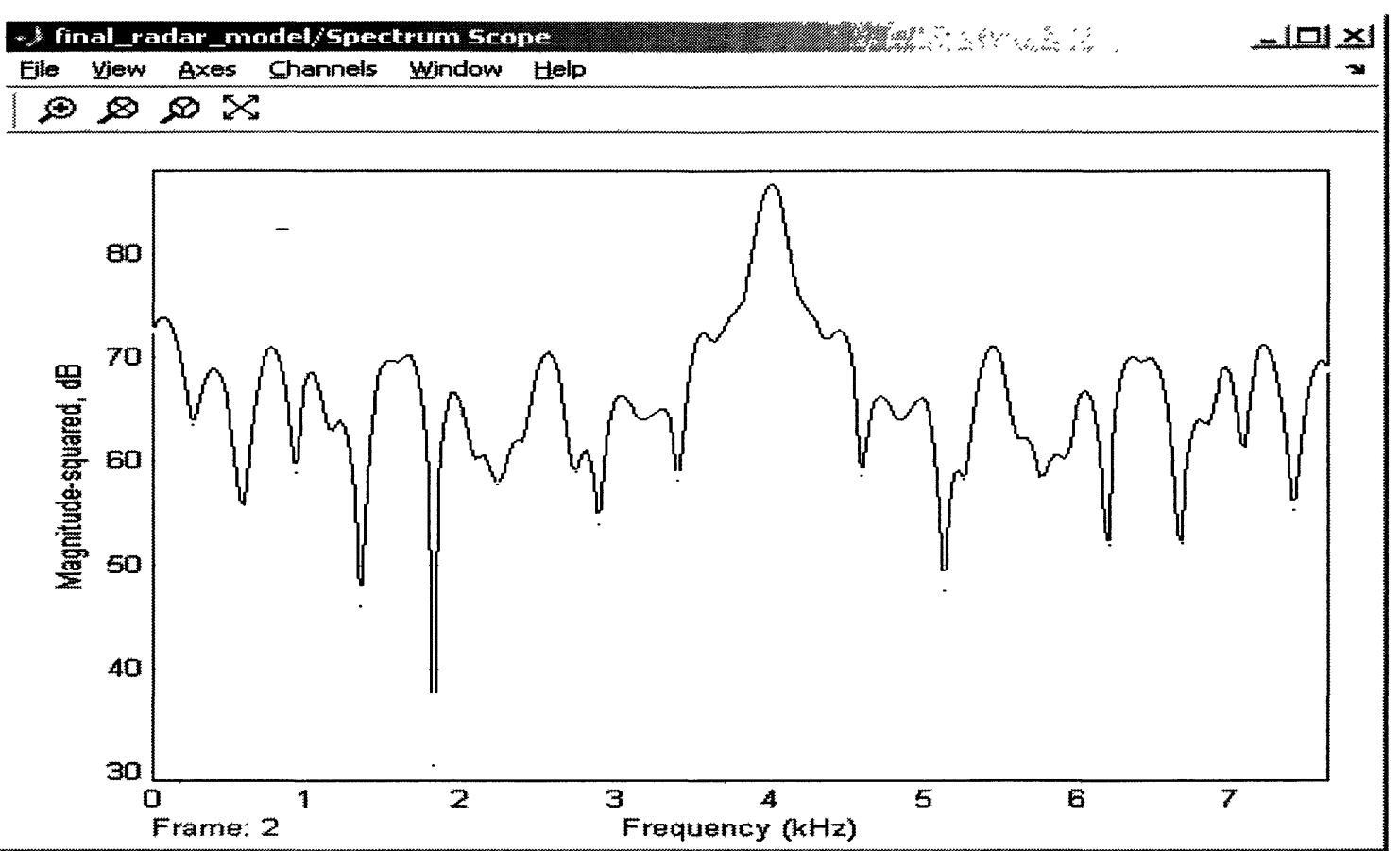

Figure 4.11: Doppler Spectrum for Swerling II target
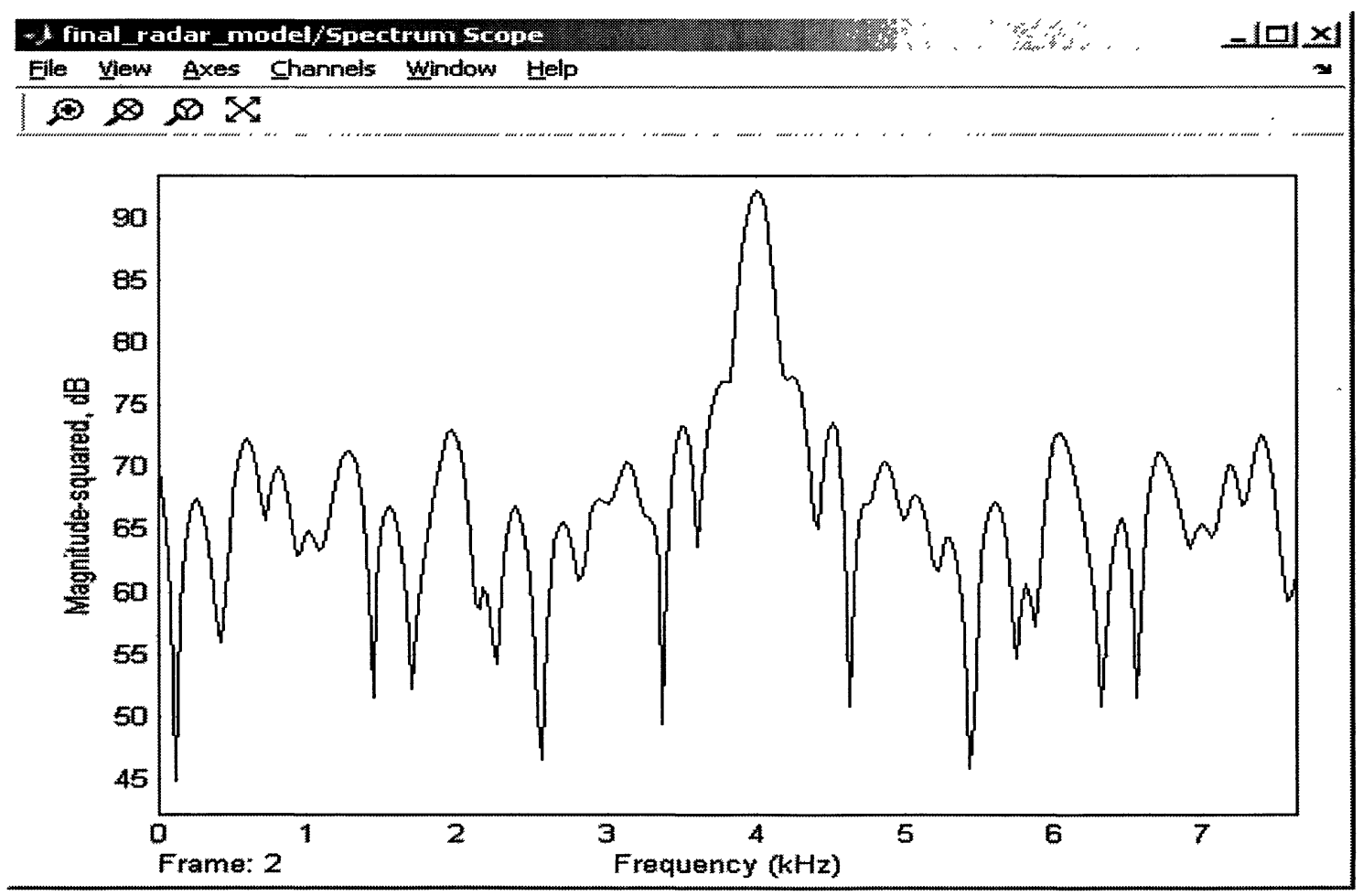

Figure 4.12: Doppler Spectrum for Swerling IV target 
Virtual clutter velocities as obtained from equation 4.2 range from approximately $0.25 \mathrm{~m} / \mathrm{s}$ to $5.047 \mathrm{~m} / \mathrm{s}$ under various sea conditions with resulting Doppler frequencies of $3.3 \mathrm{~Hz}$ to $67.3 \mathrm{~Hz}$ at a carrier frequency of $4 \mathrm{GHz}$. Figure 4.13 shows the spectrum of auto-correlation values sampled at the PRF for the clutter range bin obtained from figure 4.8. The clutter Doppler is $67.47 \mathrm{~Hz}$ as obtained from the sea state and figure 4.13 shows a peak power at this value. The Doppler frequency is computed by taking a 256 point FFT at each range bin and the Doppler shift is the frequency with the highest carrier to noise ratio.
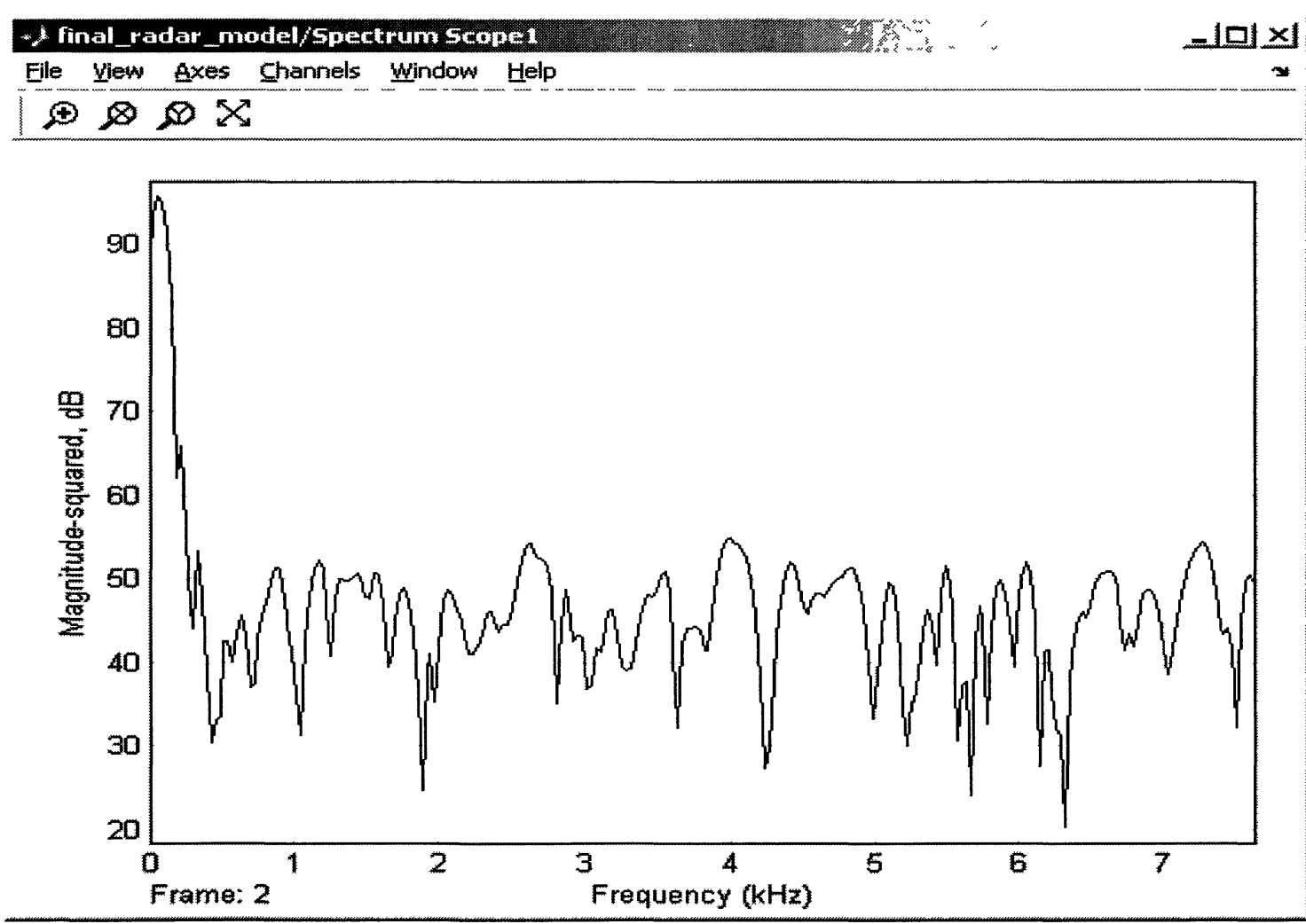

Figure 4.13: Doppler Spectrum obtained from 1024 point FFT at clutter range bin

Processing auto-correlation values to obtain the Doppler frequency improves the signal to noise ratio of the Doppler spectrum as we are processing on signals which have been 
extracted from noise. From auto-correlation values, range bins with targets or clutter are known and are used to select relevant Doppler information. Clutter can thus be rejected by setting a threshold for the minimum Doppler frequency expected and eliminating all range correlations returns below this threshold.

\subsection{Simulated Serial Vs Parallel Processing}

The determining factors for a choice between serial and parallel Doppler processing are Doppler SNR and processing speed. Parallel Radar processing implies the range and Doppler processing are done simultaneously while serial Radar processing implies range and Doppler information are extracted sequentially. Range information is obtained by correlation thus in a serial Radar process, the echo is correlated before being processed for Doppler information. Parallel Doppler processing is easily implemented in Radar Systems with waveforms which are repetitive per PRI. For such Radar Systems, the phase of the echo at each range bin is exclusively dependent on the target Doppler shift. Other Radar Systems like the one introduced in this thesis transmit different waveforms per PRI and as a result, the change in phase at each range bin is as a result of both the target Doppler shift and transmitted waveform properties. The detection of Doppler shift for such a system requires the removal of the random waveform effect before Doppler processing is carried out. The waveform proposed in this research has both frequency and phase modulated properties. The frequency modulated property is periodic and can be viewed over successive pulses without corrupting Doppler information. The phase modulated property, however, changes with each PRI implying the random nature of the phase codes has to be removed such that the target Doppler shift can be extracted. Correlation is one method of removing the random nature of 
the echoes as the auto-correlation output is consistent at the target range bin regardless of the transmitted phase code. Other random cancellation schemes require the detection of the presence of a signal and the required detection scheme should take considerably less time than it takes to correlate a signal otherwise parallel processing becomes counter-productive. Another method of removing the random nature of successive pulses is realized by taking the product of the transmitted and received waveforms at the appropriate delay (range bin). This research does not examine signal detection schemes required for parallel processing. It assumes that the radar system is able to implement signal detection with almost no delay and it examines the difference in carrier to noise ratio at the Doppler frequency between parallel and serial Radar processes. The time required for processing of a fast FFT is given by $N \log N$, where $\mathrm{N}$ is the length of the FFT. Ignoring time required for multiplication, correlation requires a processing time of $3 N \log N$ as the FFT of the transmitted and received sequences are computed as well as the inverse FFT of the complex product of said sequences. To maintain the same amount of processing time between the parallel and serial processes, the Doppler FFT length of the parallel process is chosen to be $4 N$ while the Doppler FFT length of the serial process is $N$ where $N=4096$. Figure 4.14 and 4.15 show the carrier to noise ratio at a known target delay and Doppler frequency of $100 \mathrm{~Hz}$ for serial and parallel Radar systems respectively. The simulated target is also non-fluctuating. 


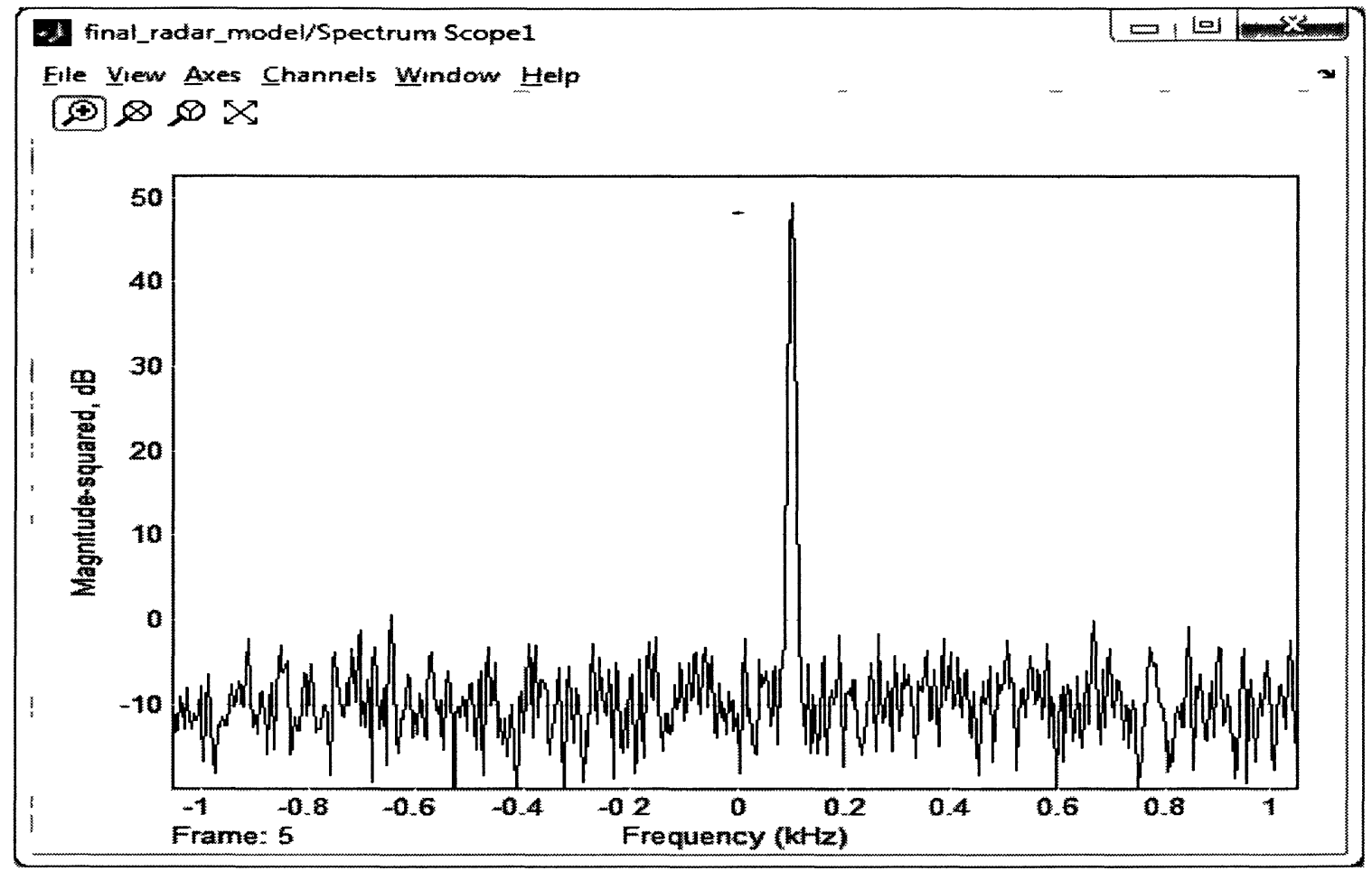

Figure 4.14: Doppler Spectrum of Serial process for non-fluctuating target

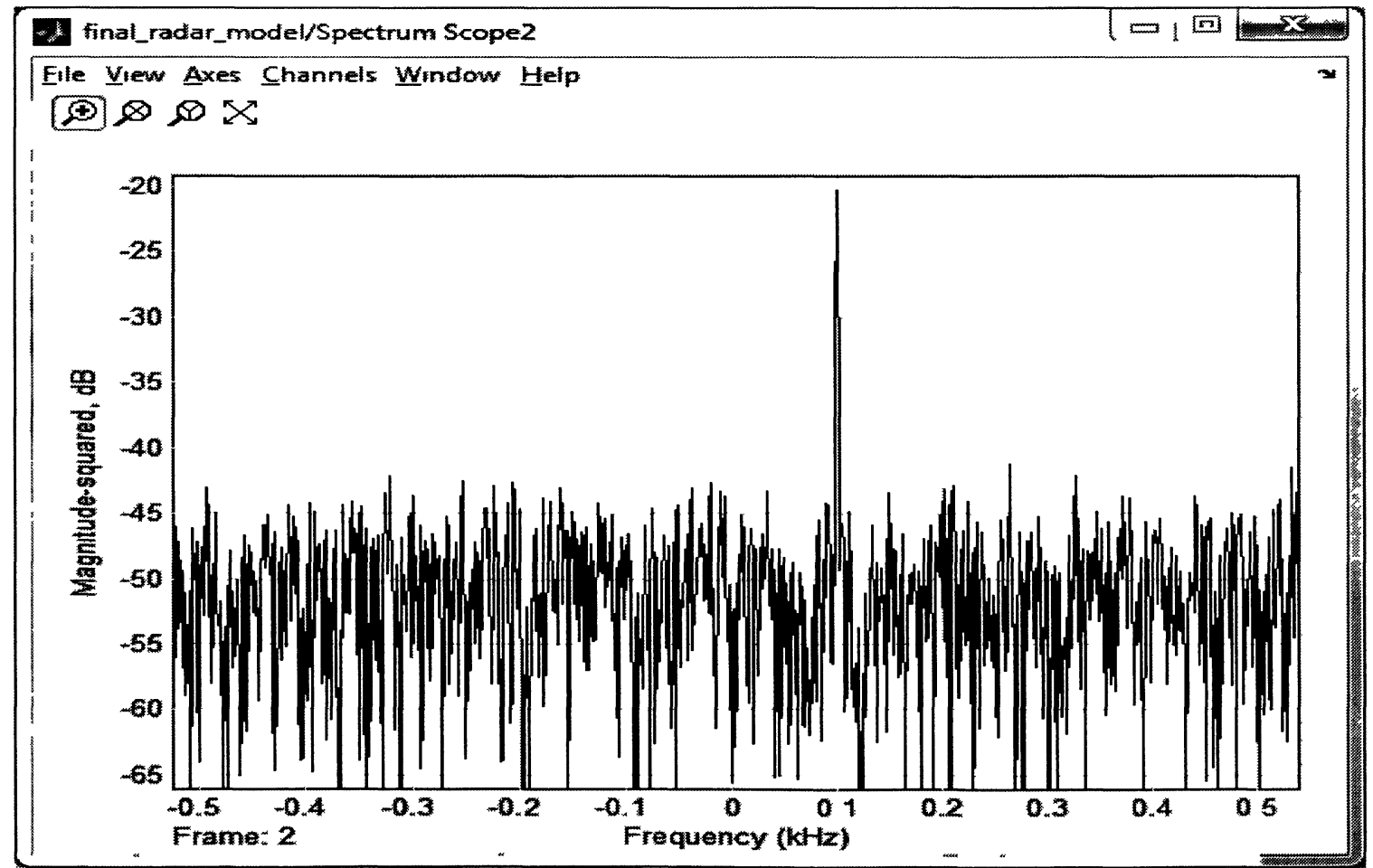

Figure 4.15: Doppler Spectrum of Parallel process for non-fluctuating target 
It can be observed from figure 4.14 that the Carrier to Noise ratio at the Doppler frequency is about $55 \mathrm{~dB}$ for the serial process while the parallel process in figure 4.15 shows a Carrier to Noise ratio of about $28 \mathrm{~dB}$. The simulated improvement factor for the serial Vs parallel process for the same processing time is $27 \mathrm{~dB}$.

\subsection{Chapter Summary}

This chapter verifies the ability of the waveform and receiver processing to detect the presence of a target in the presence of AWGN, clutter, multipath and target cross-section through Matlab/Simulink simulations. This chapter also verifies waveform range resolution by observing the delay between successive auto-correlation peaks. Finally, both serial and parallel Doppler processing is simulated in this Chapter and serial processing is observed to have about $27 \mathrm{~dB}$ improvement in CNR over parallel processing. 


\section{Chapter 5 \\ Implementation and Testing}

\subsection{Real Time Processing}

Tests carried out in this thesis are done to verify the improvement in the power requirement for systems that utilize pulse compression over systems that do not. The tests also verify target range resolution improvement of the proposed phase-coded-linear-FM waveform over phase-coded and linear-FM waveforms. Finally, the tests verify moving target detection from Doppler information and the improvement in CNR of a serial over a parallel Doppler processor. Baseband processing is implemented on a $\mathrm{PC}$ and the block diagram is shown in figure 5.1 below. The transmitted waveform generated by the convolution of a random PN sequence and a linear chirp is stored in the PC's memory before being sent to the D-TA 2300. On the receive side, FFTs on both the received and transmitted sequences are done to implement the auto-correlation function. The magnitude of the auto-correlation response is used to display target range. The received auto-correlation responses are stored in a buffer and FFT processing over successive pulses at each range bin is used to display the Doppler information. Modifications to the kernel layer of the PC were required to ensure real time processing. This important because any delay in the packet sequence of the received signal will change the range processing from auto-correlation to cross-correlation. 


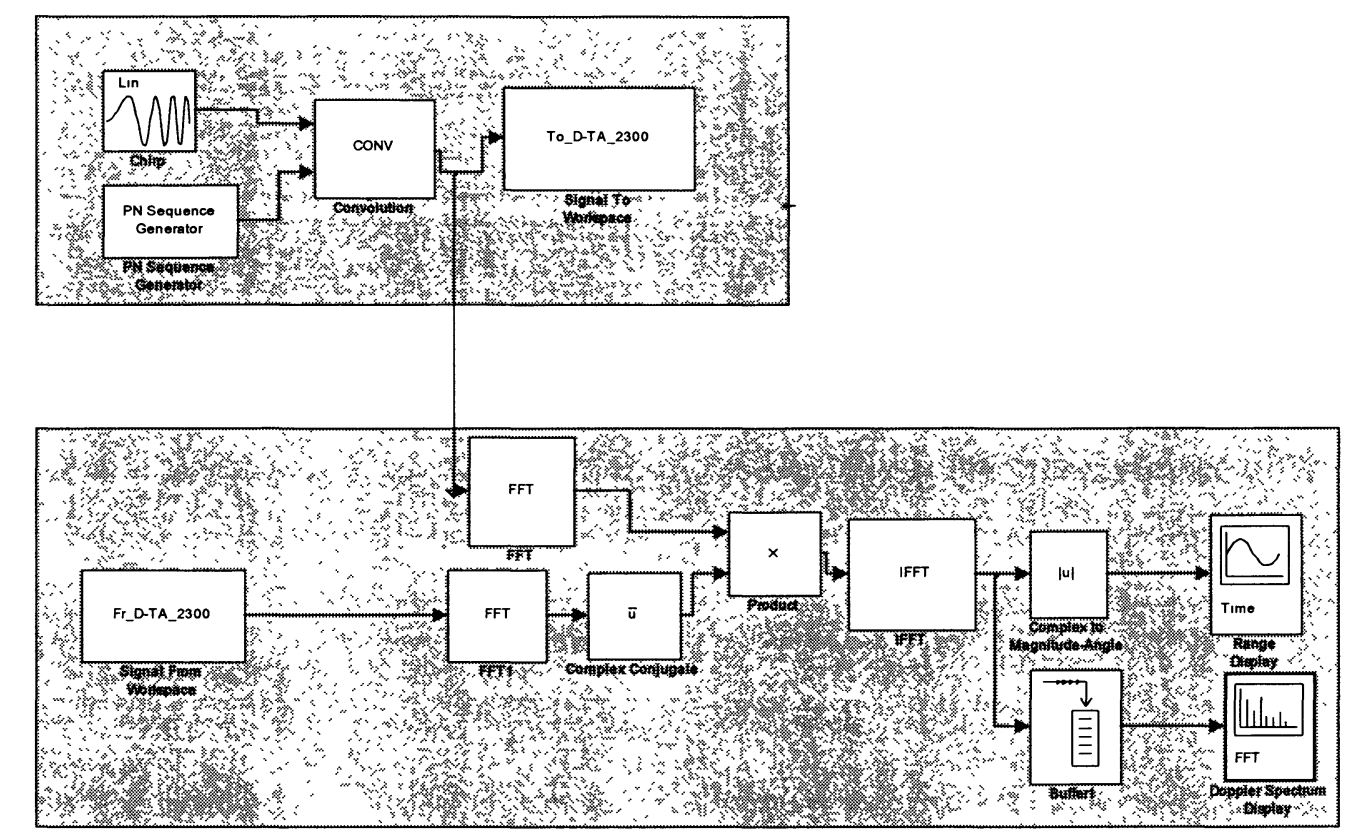

Figure 5.1: Block Diagram of Baseband Processor

\subsection{Transmitted Spectrum}

Figure 5.2 shows the spectrum of the transmitted waveform at the D-TA 2300 output as obtained by the spectrum analyzer. The D-TA 2300 convert's computer packets representing the transmitted waveform to an analog signal centered at $70 \mathrm{MHz}$ with $31.25 \mathrm{MHz}$ of bandwidth. The tested spectrum coincides with the simulated spectrum shown in figure 4.4. The only difference is the simulated spectrum is at baseband with a bandwidth of $15.625 \mathrm{MHz}$ while the tested spectrum is centered at $70 \mathrm{MHz}$ with twice the base-bandwidth of $31.25 \mathrm{MHz}$. The 70MHz IF signal is used as input to the D-TA 3290 for IF to RF upconversion. The spectrum at the output of the D-TA 3290 is the same as that in figure 5.1 except that it is centered at $1.472 \mathrm{GHz}$. This spectrum is shown in figure 5.2. 


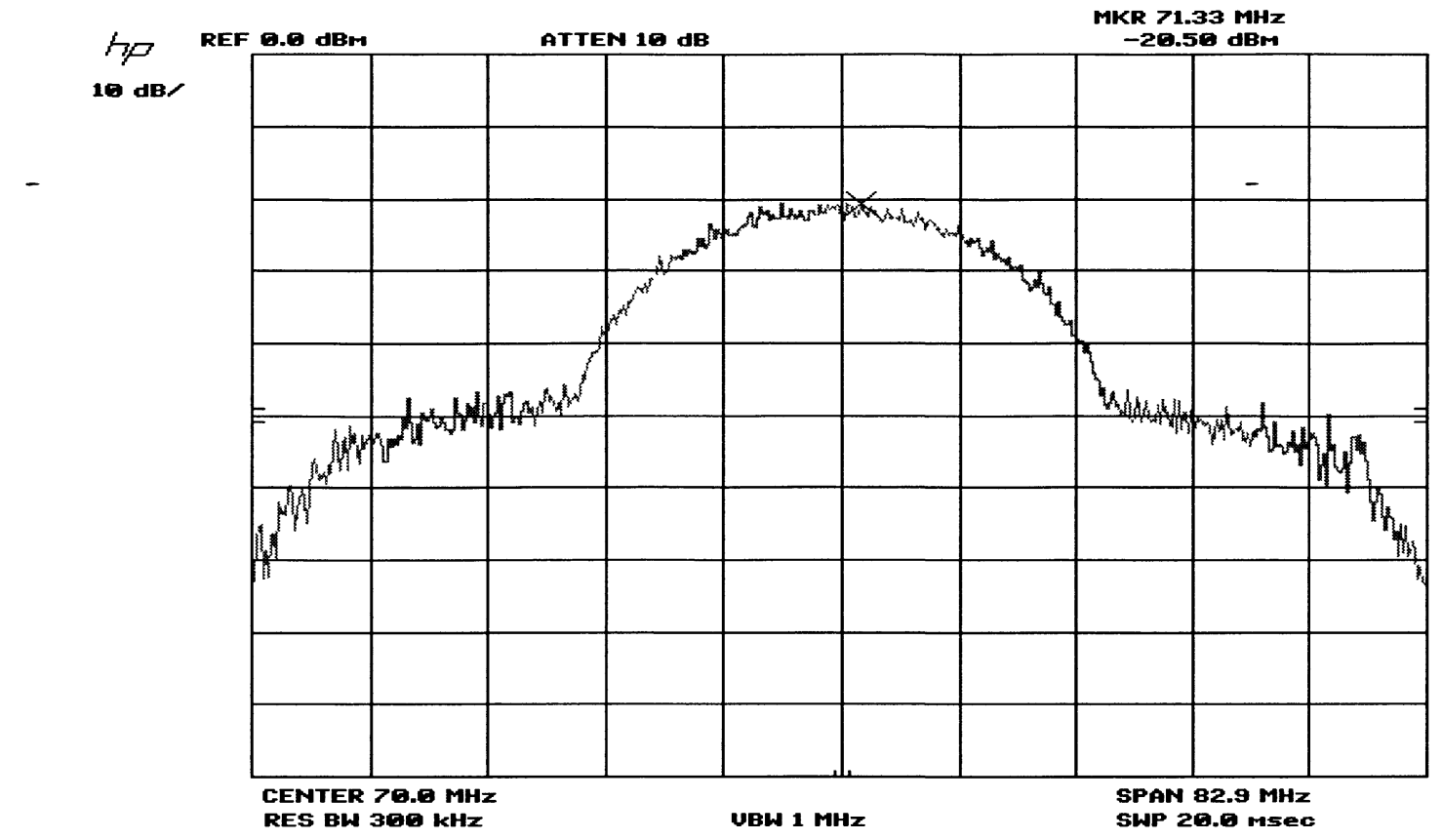

Figure 5.2: Output of D-TA 2210 as seen on Spectrum Analyzer

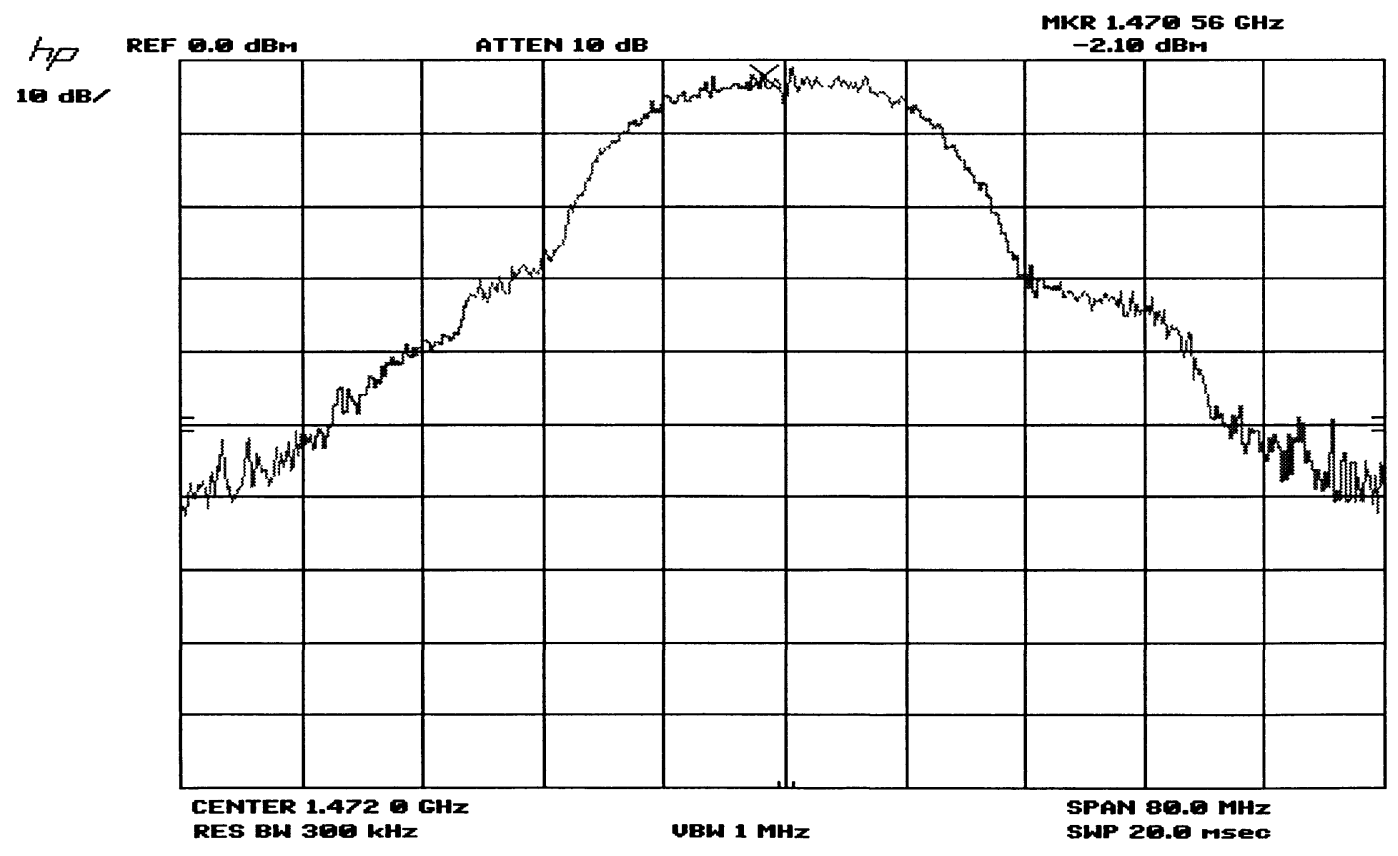

Figure 5.3: Output of D-TA 3290 as seen on Spectrum Analyzer 


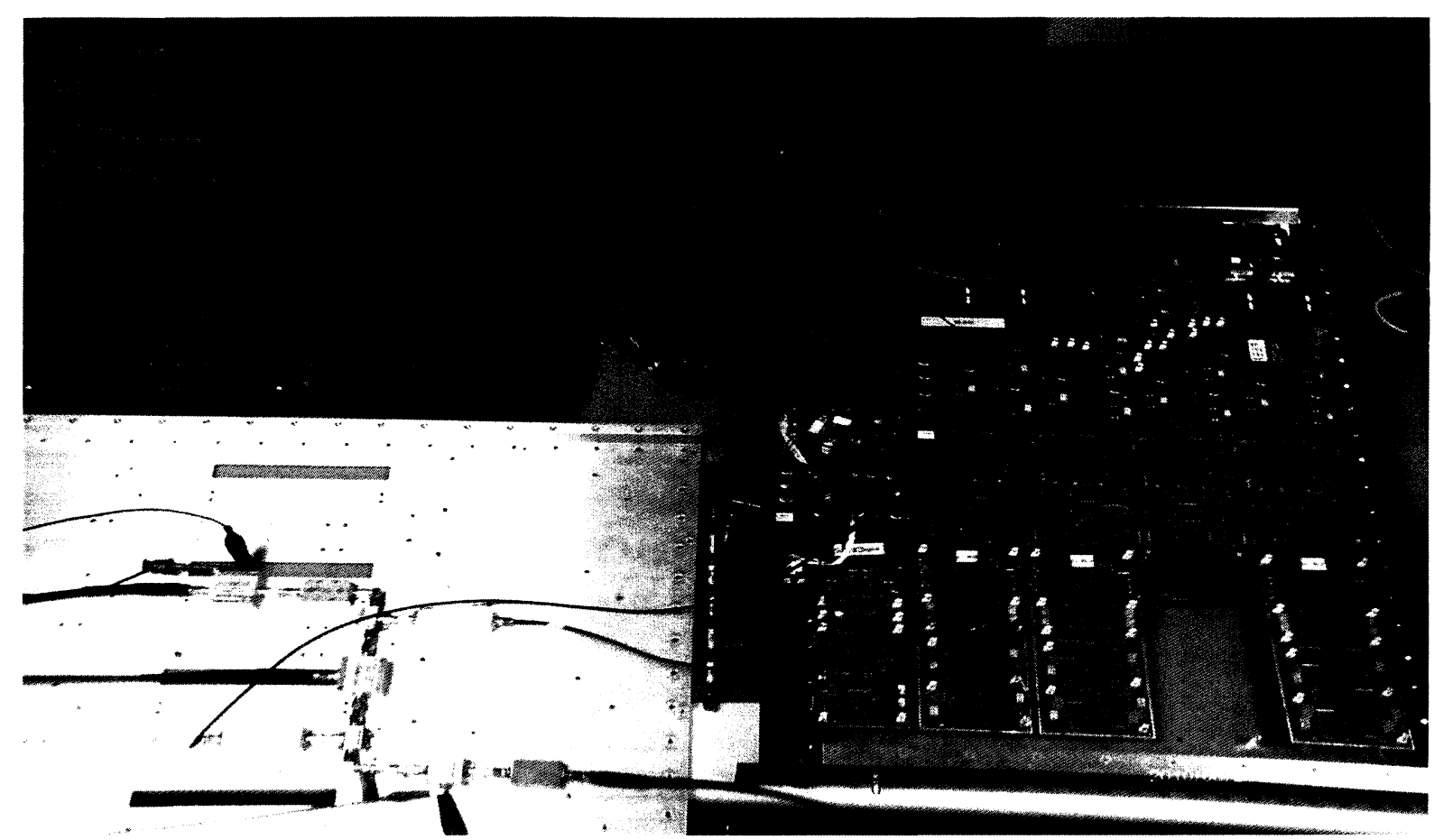

Figure 5.4: D-TA 2300 (on right) and RF transceiver (on left)
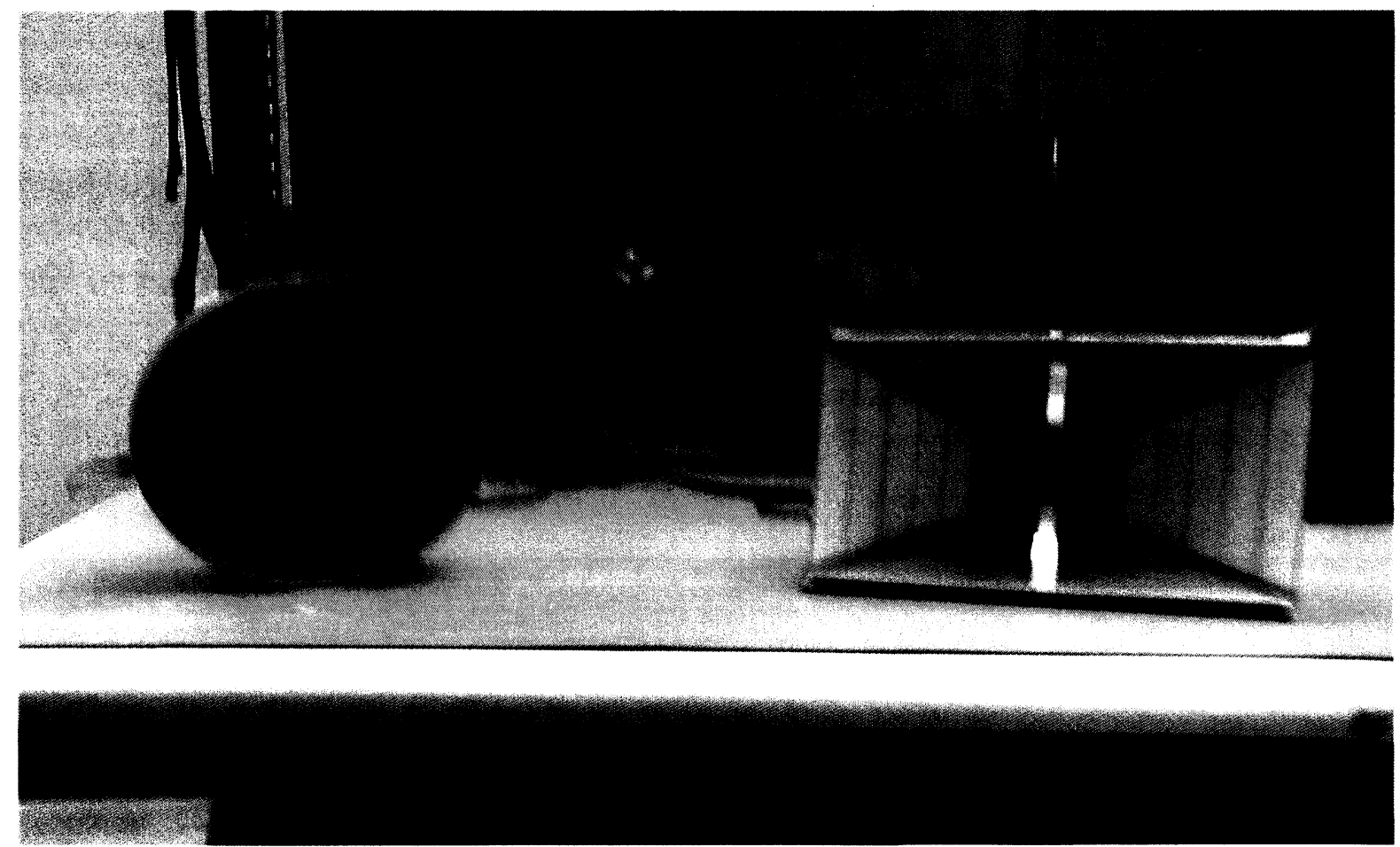

Figure 5.5: Circular polarized antenna (on left) and Horn antenna (on right) 
Figure 5.4 shows the D-TA 2300 which is used to convert computer packets to an analog signal centered at $70 \mathrm{MHz}$ and vice versa. It also shows the RF transceiver (on the left) which is used to up-convert and down-convert signals from $70 \mathrm{MHz}$ to $1.5 \mathrm{GHz}$ and $1.5 \mathrm{GHz}$ to $70 \mathrm{MHz}$ respectively. Figure 5.5 shows the horn antenna and the circular polarized antenna used to transmit and receive signals respectively.

\subsection{Resolution and Power Verification}

The proposed waveform resolution is verified by detection of a rectangular metal object in an office environment over various ranges for all three types of waveforms. The antenna used for transmission is the H-1734 manufactured by BAE Systems. The H-1734 is a linear polarized horn antenna with $8.65 \mathrm{~dB}$ of bore sight gain at $1.5 \mathrm{GHz}$. Figure 6.1 and 6.2 under Appendix A show the antenna gain variation with target angle for the elevation and horizontal planes respectively. The receiving antenna is the ASO-1658A which is a circular polarized antenna with $2 \mathrm{~dB}$ of bore sight gain at $1.5 \mathrm{GHz}$ [15]. Since the transmitting antenna is linear polarized and the receiving antenna is cross-polarized, there is a $3 \mathrm{~dB}$ loss in antenna gain. This implies the antenna gain to be used in the range equation (1.10) is given by:

$G=G_{t}+G_{r}-6 d B=4.65 d B$. The full system test is done in a closed stationery environment and the transmit power can be observed from figure 5.3 to be $-2.1 \mathrm{dBm}$. Given the above transmit power and antenna parameters used to conduct the experiment, the expected maximum ranges for a single return with and without processing gain can be calculated and the resulting values compared to the maximum range obtained from 
correlation. Let the minimum target cross-section $(\sigma)$ be $1 \mathrm{~m}^{2}, k T_{o}=-174 \mathrm{dBm} / \mathrm{Hz}$ and minimum SNR per pulse $-\left(\frac{s}{N}\right)_{1}=6.02 \mathrm{~dB}$. Since there is no pulse integration, $n=1$. The Noise Bandwidth $\left(B_{n}\right)$ and Noise Factor $\left(F_{n}\right)$ are system parameters of the D-TA 3290 and are given by $40 \mathrm{MHz}$ and 10 respectively. Maximum range without correlation gain is obtained from equation 2.10 and is calculated as follows:

$R_{\max }^{4}=\frac{0.617 m * 2.92^{2} * 0.204^{2}}{(4 \pi)^{3} *(3.981 E-21) * 40 E 6 * 10 * 4}=>R_{\max }=11.45 \mathrm{~m}$

Maximum range with correlation gain is obtained from equation 2.14 and is calculated as follows:

$R_{\max }^{4}=\frac{0.617 m * 2.92^{2} * 1024 * 0.204^{2}}{(4 \pi)^{3} *(3.981 E-21) * 40 E 6 * 10 * 4}=>R_{\max }=64.76 \mathrm{~m}$

Based on calculations, a waveform with pulse compression can achieve a maximum range of about 5.6 times the maximum range of a waveform without pulse compression. Due to test environment limitations, the maximum range the target was able to reach was $9.8 \mathrm{~m}$.

However, the maximum range which can be obtained from auto-correlation can be observed in figure 5.6. Figure 5.6 shows no returns above $40 \mathrm{~m}$ and this value can be viewed as the maximum range the system is able to process. The observed $R_{\max }$ of 40 is smaller than the calculated $64.76 \mathrm{~m}$ value and this could be due to a smaller cross-section at the maximum range than the estimated $1 m^{2}$ value. The observed $R_{\text {max }}$ is however greater than the calculated $R_{\text {max }}$ for a transmission scheme without pulse compression $(11.45 \mathrm{~m})$ thus validating the premise that pulse compression can reduce the required transmitter power. 
Figures 5.6, 5.7 and 5.8 show range plots for the phase-coded-linear-FM waveform, phase coded waveform and linear FM waveforms respectively. Table 5.1 shows normalized autocorrelation magnitudes of all three waveforms over various distances. Each figure shows range returns for the target at two separate resolution bins where each resolution bin is $2.4 \mathrm{~m}$. Given a $31.25 \mathrm{MHz}$ system bandwidth, the phase coded waveform is only able to achieve $9.8 \mathrm{~m}$ of range resolution and this is observed by the superimposition of the range returns shown in figure 5.5. The phase-coded-linear-FM signal is able to achieve the same range resolution as the linear-FM waveform $-2.4 \mathrm{~m}$ as shown in figure 5.6 and 5.8 respectively. In this way, the range resolution advantage of frequency modulation is verified.

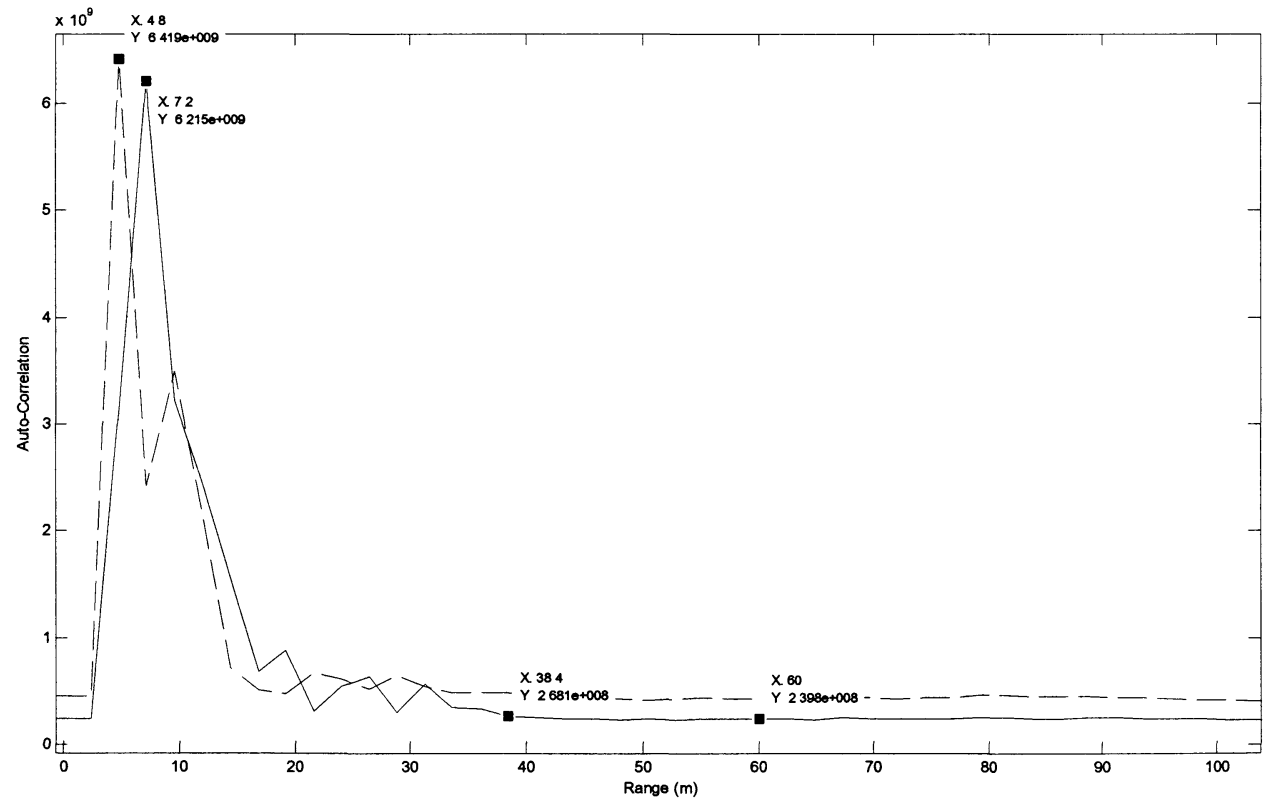

Figure 5.6: Plot of Auto-correlation against range for phase-coded-linear-FM waveform 


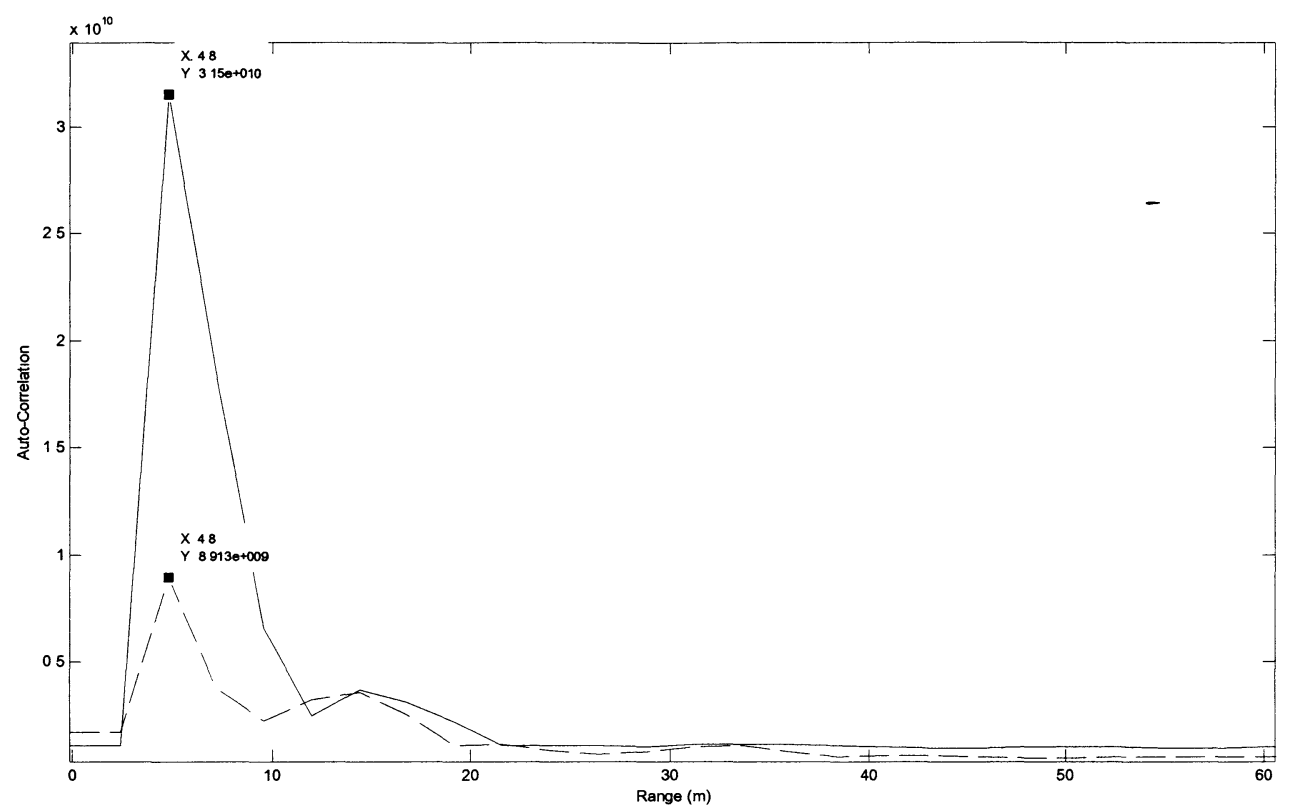

Figure 5.7: Plot of Auto-correlation against range for phase-coded waveform

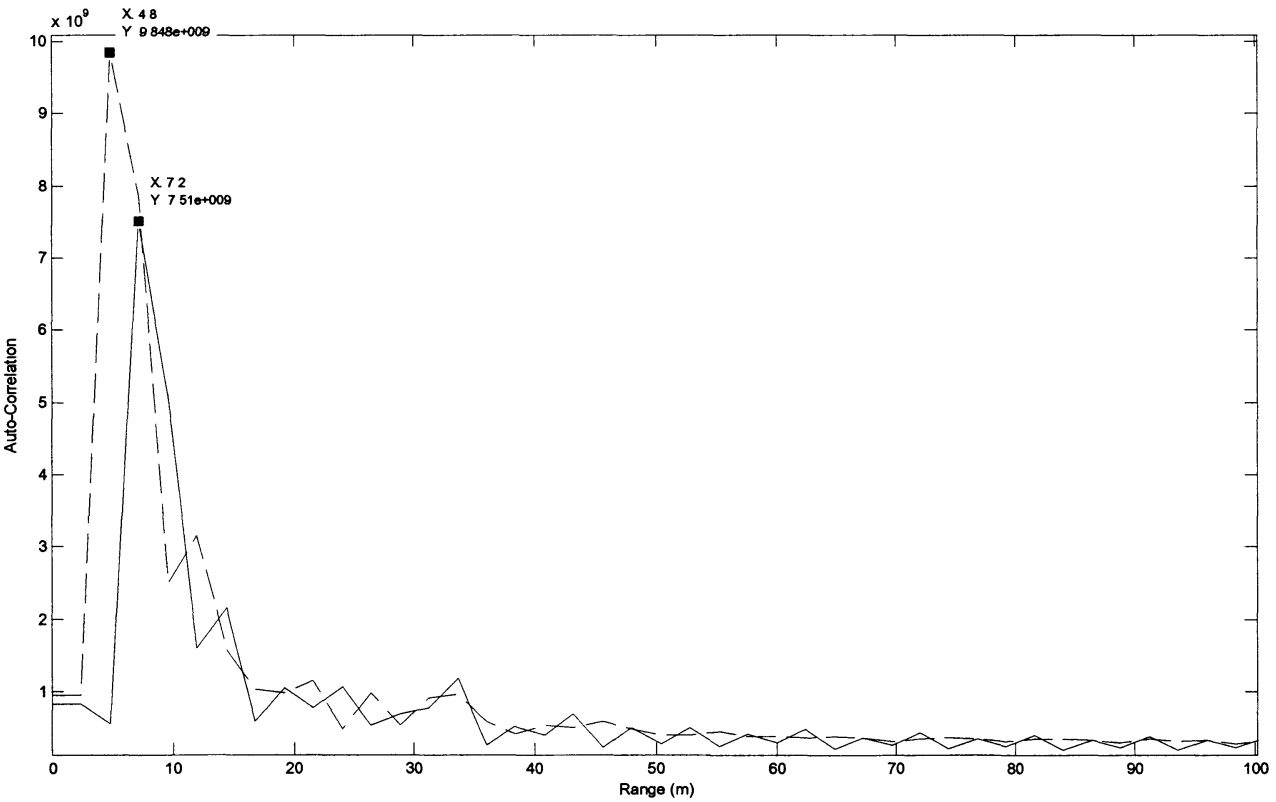

Figure 5.8: Plot of Auto-correlation against range for linear-FM waveform 


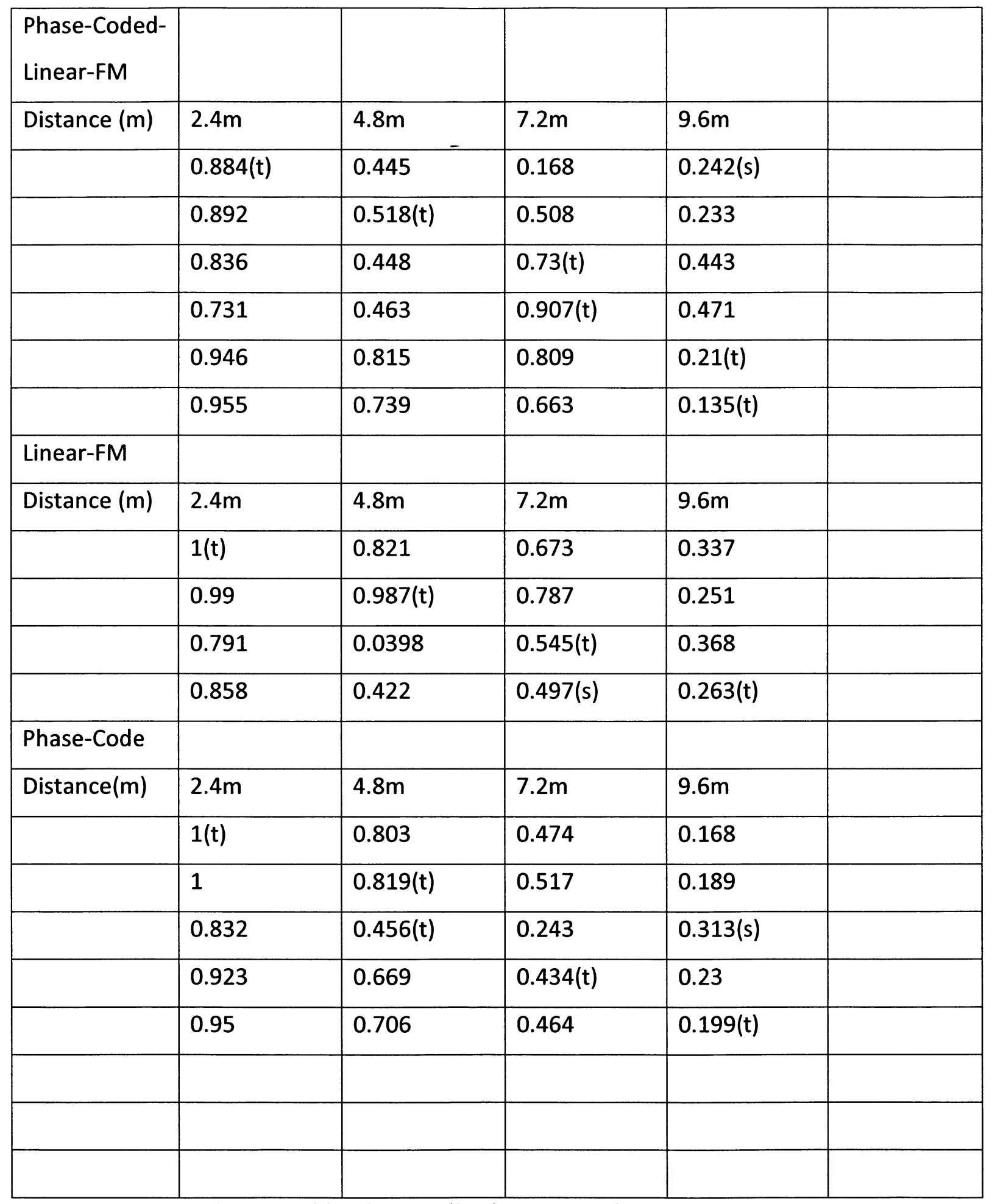

Table 5.1: Table of Normalized Auto-correlation values 
Table 5.1 shows the normalized auto-correlation values for all three types of waveforms at various distances or range bins. Values in table 5.1 with $(t)$ next to them imply the location of the metal box target while values with (s) imply correlation side-lobes. Due to the high clutter nature of the environment, returns exist at each range bin and the metal box can only be clearly visualized at certain range bins. For both the phase-modulated-linear-FM and linear-FM signals, the metal box auto-correlation peak is clearly visualized within the $7.2 \mathrm{~m}$ resolution bin. The phase-coded waveform only shows an auto-correlation peak within the $4.8 \mathrm{~m}$ resolution bin due to the $9.8 \mathrm{~m}$ resolution property of the waveform.

\subsection{Doppler Verification}

\subsubsection{Doppler Test Setting}

The ability of the radar system to distinguish stationery from moving targets depends on the Doppler shift moving targets cause. The moving target used in this experiment is a Powermax fan with Aluminum blades. The fan has four blades and each blade has a radius of $0.1778 \mathrm{~m}$. The transmitted signal hits the rotating blades which are moving perpendicular to the direction of the signal. Figure 5.9 shows the cross-section diagram of the Powermax fan blades. The echo at the receiver is a signal shifted by a Doppler which is proportional to the radial velocity of the fan blades. Since radial velocity is directly proportional to the distance from the center of the fan, each portion of the blade adds a Doppler shift which depends on its distance from the center of the fan. This problem is minimized by using fan blades with 
minimal cross-section around the center but increase as the fan edges are approached. Radial velocity can be calculated with knowledge of the fan rpm as follows:

$l_{\text {arc }}=r \theta=>$

$v_{r}=\frac{d l_{\text {arc }}}{d t}=r \frac{d \theta}{d t}=2 \pi r(r p m) / 60$

Where $v_{r}$ the radial velocity, $\mathrm{r}$ is the distance from the center to any point in the blade and $l_{\text {arc }}$ is the length of the arc made by any point on the blade as defined by its radius. Fan speed is tested at two values of rpm -1220 and 1517. From the above equation, the radial velocity at the edge of the fan with radius of $0.1778 \mathrm{~m}$ is calculated as follows:

$$
\begin{aligned}
& v_{r_{-} \min }=2 \pi * \frac{0.1778(1220)}{60}=\frac{22.715 \mathrm{~m}}{\mathrm{~s}} \\
& v_{r_{-} \max }=2 \pi * \frac{0.17(1517)}{60}=28.245 \mathrm{~m} / \mathrm{s}
\end{aligned}
$$

The resulting Doppler frequencies are obtained from $f_{d}=v_{r} * \frac{f_{c}}{c}$ and given a carrier frequency of $1.472 \mathrm{GHz}$, the minimum and maximum Doppler frequencies are calculated as follows:

$$
\begin{aligned}
& f_{d \_ \text {min }}=21.71 * \frac{1.472 E 9}{3 E 8}=111.46 \mathrm{~Hz} \\
& f_{d \_ \text {max }}=27.01 * \frac{1.472 E 9}{3 E 8}=138.59 \mathrm{~Hz}
\end{aligned}
$$

The calculated Doppler frequencies are as a result of velocities at the edge of the fan blades; however, the maximum cross-section of the fan occurs at about half the length of the blades. Since the cross-section is what dictates the strength of the echo, the most dominant Doppler shift will occur at the point with the highest cross-section. This implies that the observable 
minimum and maximum Doppler shifts will be about half the calculated values or $55.728 \mathrm{~Hz}$ and $69.295 \mathrm{~Hz}$ for $1220 \mathrm{rpm}$ and $1517 \mathrm{rpm}$ respectively.

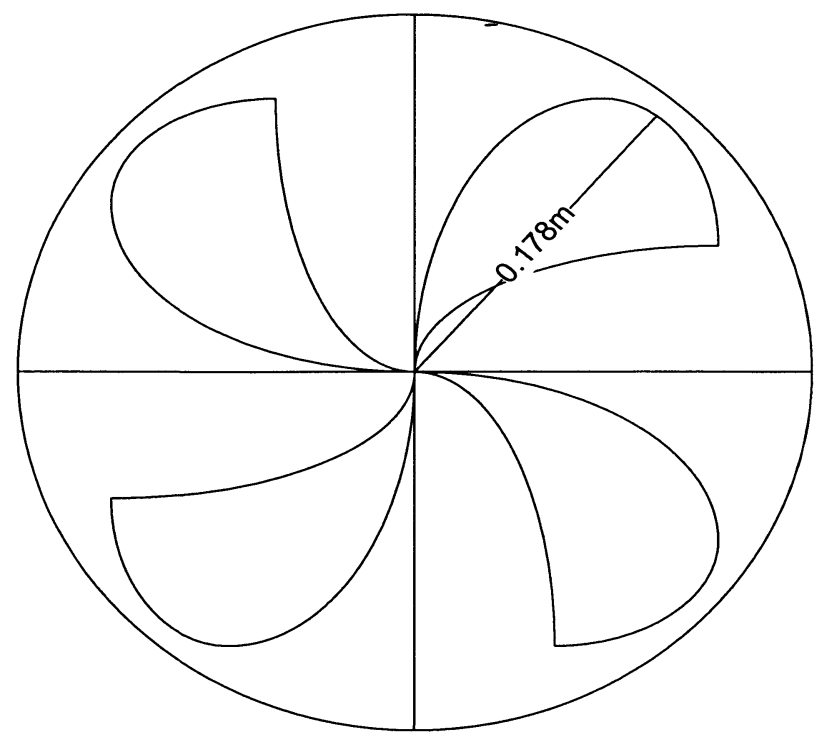

Figure 5.9: Cross-Section of Powermax fan

\subsubsection{Doppler Test Results}

Serial Doppler processing is done by taking the FFT of correlated values over successive echoes at each range bin. The magnitude of the complex correlated values gives an indication of the target range while the phase of the complex correlated values gives an indication of the Doppler shift. Correlation improves the carrier to noise ratio (CNR) of the return while preserving the phase such that fewer pulses are required to obtain the desired Doppler carrier to noise ratio. To improve the correlation gain, the pulse repetition interval was increased by a factor of 7 yielding a pulse repetition frequency (PRF) of $2.18 \mathrm{kHz}$. Due to real time processing restrictions, the maximum FFT length is 256 yielding a Doppler resolution of 
8.5Hz. Figure 5.10 and 5.11 show the Doppler spectra at the fan range bin for rotating speeds of $1220 \mathrm{rpm}$ and $1517 \mathrm{rpm}$ respectively.

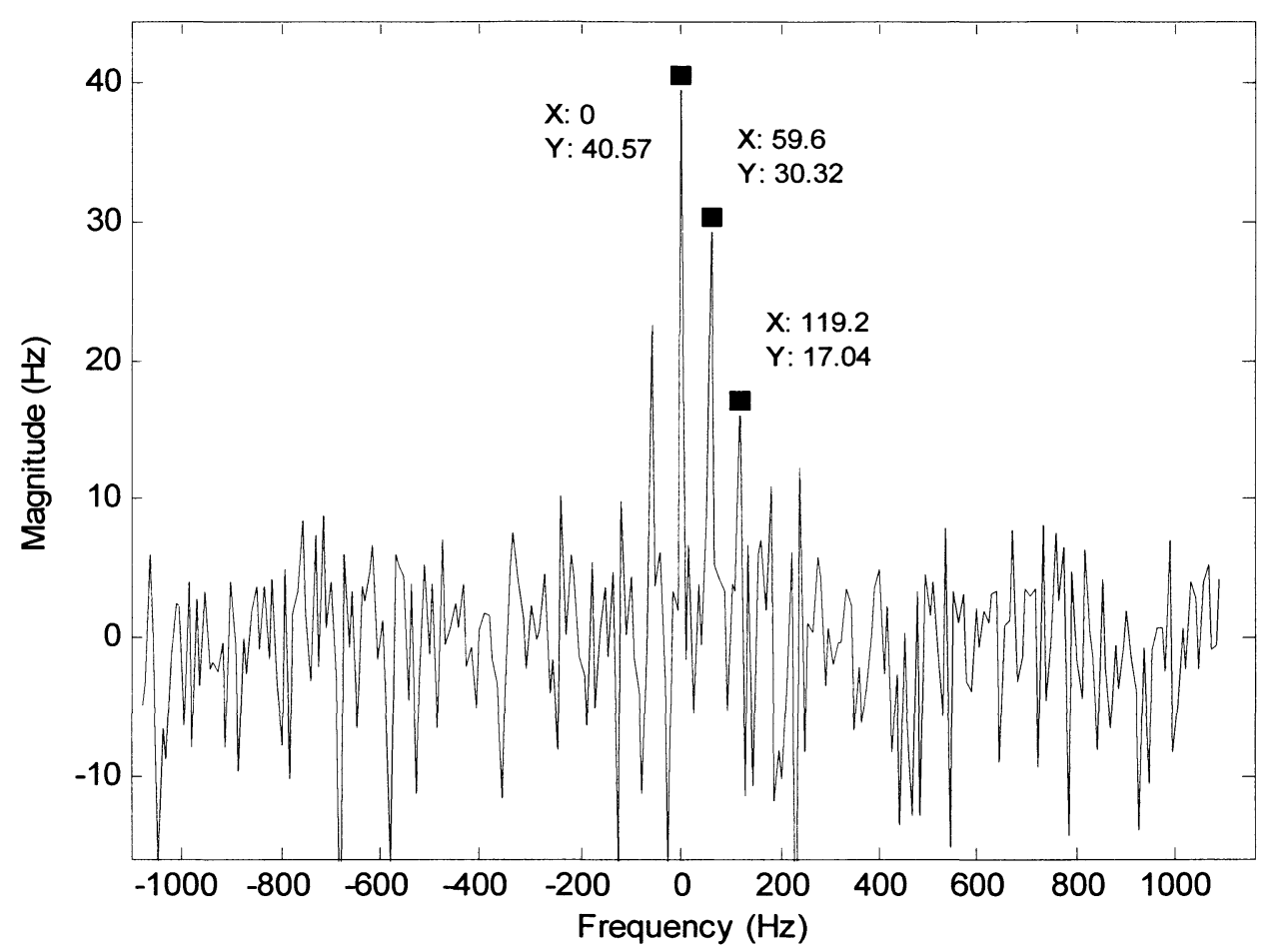

Figure 5.10: Doppler spectrum at target range bin for fan rotating at 1220rpm

Figure 5.10 shows frequency components at DC, $59.6 \mathrm{~Hz}$ and $119.2 \mathrm{~Hz}$. The DC component is as a result of the stationery clutter present at the same range bin as the fan. The components at $59.6 \mathrm{~Hz}$ and $119.2 \mathrm{~Hz}$ correspond to Doppler shifts produced by the center and edge radial velocities respectively. The calculated Doppler shift due to the center radial velocity is $55.728 \mathrm{~Hz}$ implying a discrepancy of $3.872 \mathrm{~Hz}$. Since the FFT resolution is $8.5 \mathrm{~Hz}$, the discrepancy falls within the FFT resolution bin. The calculated Doppler shift due to the edge radial velocity is $111.46 \mathrm{~Hz}$ implying a discrepancy of $7.74 \mathrm{~Hz}$. 


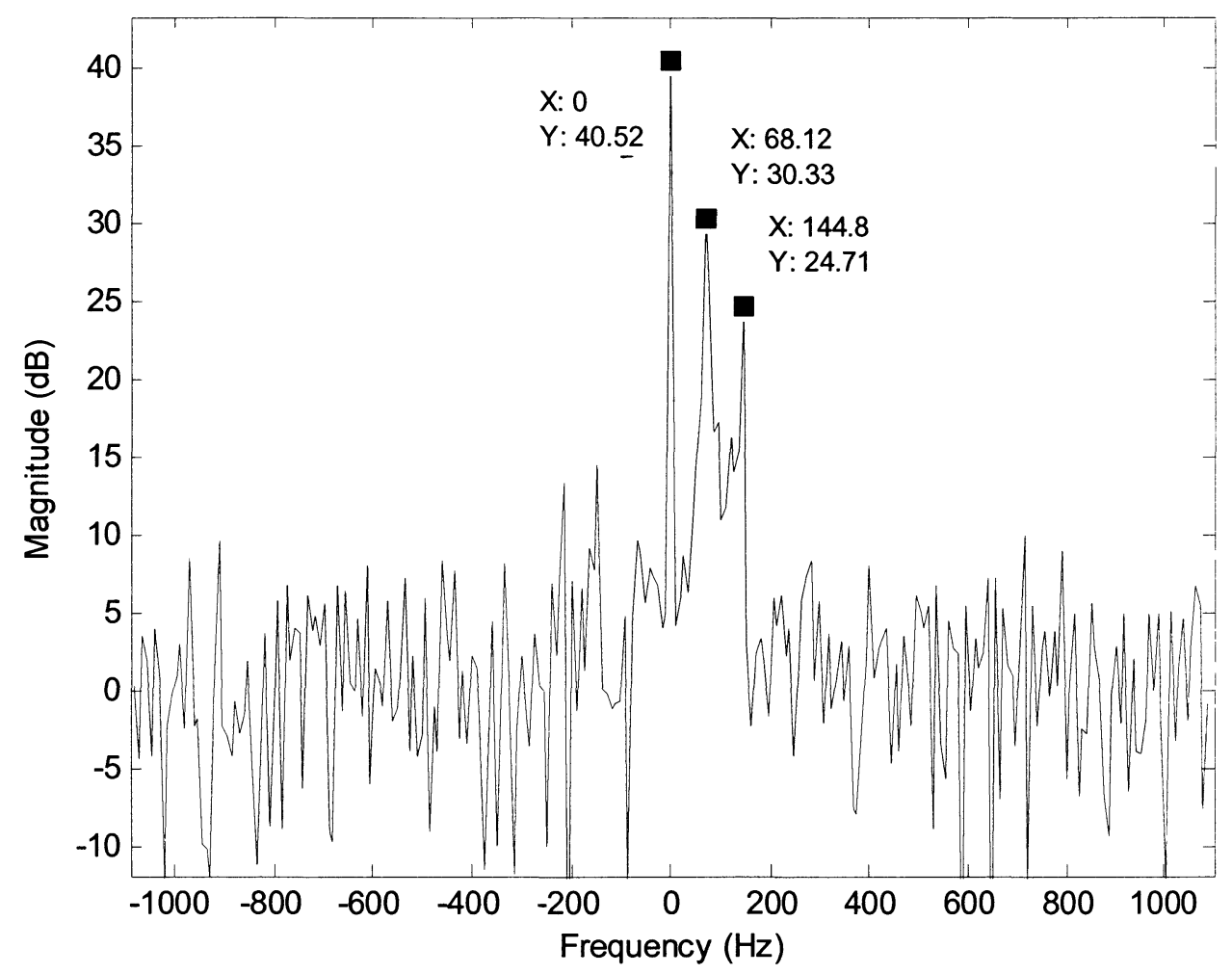

Figure 5.11: Doppler spectrum at target range bin for fan rotating at $1517 \mathrm{rpm}$

Figure 5.11 shows frequency components at DC, $68.12 \mathrm{~Hz}$ and $144.8 \mathrm{~Hz}$. The DC component is again as a result of the stationery clutter while the components at $68.12 \mathrm{~Hz}$ and $144.8 \mathrm{~Hz}$ correspond to Doppler shifts produced by the center and edge radial velocities respectively. The calculated Doppler shift due to the center radial velocity is $69.295 \mathrm{~Hz}$ implying a discrepancy between measured and calculated values of $1.175 \mathrm{~Hz}$ which falls within the FFT resolution bin. The calculated Doppler shift due to the edge radial velocity is $138.59 \mathrm{~Hz}$ implying a discrepancy of $6.21 \mathrm{~Hz}$. 


\subsection{Tested Serial Vs Parallel Radar Processing}

Experimental testing of the parallel process is done by multiplying the received sequence from the rotating (fan) target with the transmitted sequence. Figure 5.12 shows both serial and parallel processed Doppler spectra at the target range bin for the fan rotating at 1220rpm. The serial Doppler spectrum is obtained by computing the FFT over 256 pulses while the parallel Doppler spectrum is obtained by computing the FFT over 1024 pulses. Since 1024 pulses are utilized for the parallel process, the FFT length used to obtain the Doppler spectrum is 1024 and the resulting frequency resolution is:

$\frac{P R F}{1024}=\frac{15.26 \mathrm{k}}{1024}=14.9 \mathrm{~Hz}$. For the parallel Doppler spectrum shown in figure 5.12, the spectrum peak occurs at DC with a CNR of $33.19 \mathrm{~dB}$. The next predominant peak occurs at $119.2 \mathrm{~Hz}$ with a CNR of $17.57 \mathrm{~dB}$. The observed edge Doppler shift coincides with that obtained from serial processing and differs from the calculated Doppler shift of $111.46 \mathrm{~Hz}$ by 7.74Hz. The CNR at DC as obtained by the serial process is $51.01 \mathrm{~dB}$ while the CNRs at the edge and center Doppler frequencies of the fan are $27.4 \mathrm{~dB}$ and $38 \mathrm{~dB}$ respectively as observed from figure 5.12. Figure 5.13 shows both serial and parallel processed Doppler spectra at the target range bin for the fan rotating at $1517 \mathrm{rpm}$ as obtained from 256 pulses for the serial process and 1024 pulses for the parallel. For the parallel Doppler process, spectrum peaks again occurs at DC and $149 \mathrm{~Hz}$ with CNRs of $33.6 \mathrm{~dB}$ and $25.03 \mathrm{~dB}$ respectively as observed in figure 5.13. The CNR at DC as obtained from the serial process is $51 \mathrm{~dB}$ while the CNRs at fan Doppler shifts of $68.12 \mathrm{~Hz}$ and $144.8 \mathrm{~Hz}$ are $38.39 \mathrm{~dB}$ and $30.36 \mathrm{~dB}$ respectively as observed from figure 5.13. 

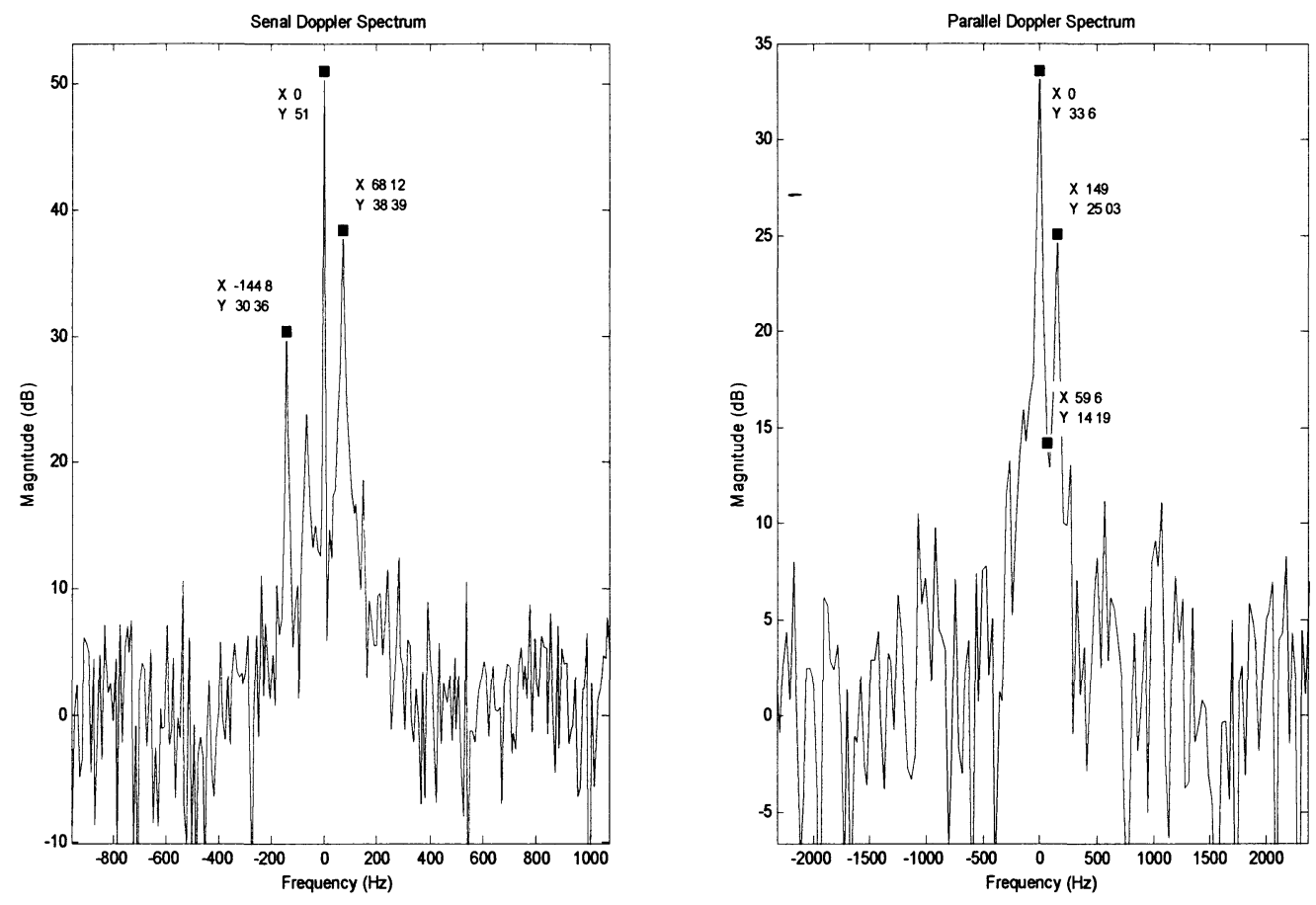

Figure 5.12: Serial and Parallel Doppler Spectrum of fan at 1220rpm
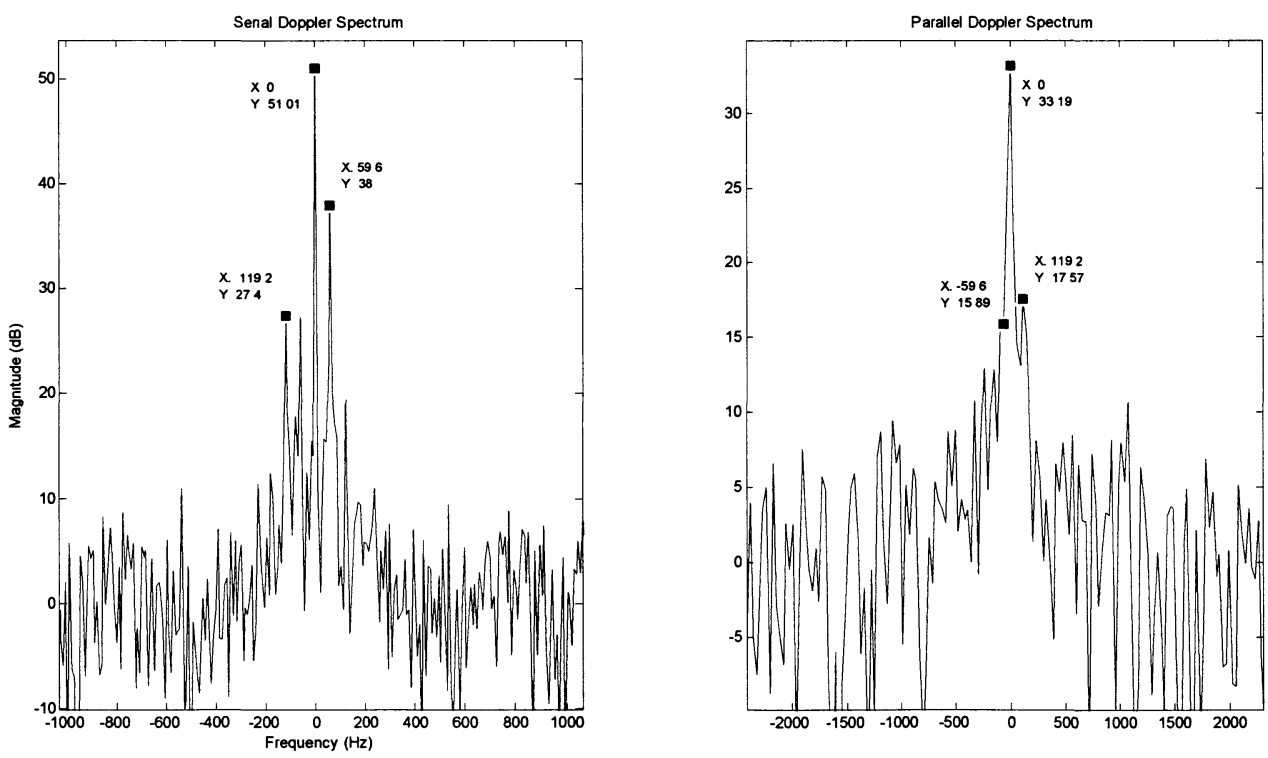

Figure 5.13: Serial and Parallel Doppler Spectrum of fan at 1517rpm 


\begin{tabular}{|l|l|l|l|l|l|l|l|l|}
\hline $\begin{array}{l}\text { Calculat } \\
\text { ed } \\
\begin{array}{l}\text { Doppler } \\
(\mathrm{Hz})\end{array}\end{array}$ & $\begin{array}{l}\text { Tested } \\
\text { Serial } \\
\text { Frequen } \\
\text { cy }(\mathrm{Hz})\end{array}$ & $\begin{array}{l}\text { Tested } \\
\text { Parallel }\end{array}$ & $\begin{array}{l}\text { Simulat } \\
\text { ed } \\
\text { ncy }(\mathrm{Hz}) \\
-\end{array}$ & $\begin{array}{l}\text { Simulat } \\
\text { ed } \\
\text { Parallel } \\
\text { Serial } \\
\text { CNR } \\
(\mathrm{dB})\end{array}$ & $\begin{array}{l}\text { Simulate } \\
\text { d CNR } \\
\text { Improve } \\
\text { ment } \\
(\mathrm{dB})\end{array}$ & $\begin{array}{l}\text { Tested } \\
\text { Serial } \\
\text { CNR } \\
(\mathrm{dB})\end{array}$ & $\begin{array}{l}\text { Tested } \\
\text { Parallel } \\
\text { CNR } \\
(\mathrm{dB})\end{array}$ & $\begin{array}{l}\text { Tested } \\
\text { CNR } \\
\text { Improve } \\
\text { ment } \\
(\mathrm{dB})\end{array}$ \\
\hline 0 & 0 & 0 & 55 & 28 & 27 & 51.01 & 33.19 & 17.82 \\
\hline 55.728 & 59.6 & N/A & 55 & 28 & 27 & 38 & 15.89 & 22.11 \\
\hline 111.46 & 119.2 & 119.2 & 55 & 28 & 27 & 27.4 & 17.57 & 9.83 \\
\hline 69.295 & 68.12 & N/A & 55 & 28 & 27 & 38.39 & 17.63 & 20.76 \\
\hline 138.59 & 144.8 & 149 & 55 & 28 & 27 & 30.36 & 25.03 & 5.33 \\
\hline
\end{tabular}

Table 5.2: Tested and Simulated Serial and Parallel carrier to noise ratios at different frequencies

Table 5.2 shows the CNRs for both serial and parallel Doppler processes at all Doppler frequencies as well as the CNR improvement ratio between these two processes.

\subsection{Chapter Summary}

This chapter describes the implementation of the baseband processor on a PC to ensure real time processing. Based on the described implementation, the proposed waveform is experimentally transmitted and received and the resulting auto-correlation response is examined to verify range resolution and power optimization. Finally, the Doppler Spectrum of a rotating fan at $1220 \mathrm{rpm}$ and $1517 \mathrm{rpm}$ is examined for both parallel and serial Doppler processes with the highest serial to parallel improvement ratio of $20.76 \mathrm{~dB}$ observed at $69.25 \mathrm{~Hz}$. 


\section{Chapter 6 \\ Discussion and Conclusion}

\subsection{Discussion}

As shown in figure 5.6, the peak auto-correlation value for the phase-coded-linear-FM waveform occurs at $7.2 \mathrm{~m}$ and table 5.1 verifies this with a peak normalized auto-correlation value of 0.907 . Since targets at or below the smallest range bin $(2.4 \mathrm{~m})$ are overshadowed by the main beam interference, auto-correlation values at this range bin do not reflect the presence of a target. From table 5.1, if the target is at $4.8 \mathrm{~m}$ and the auto-correlation values at the $2.4 \mathrm{~m}$ range bin are ignored, a peak normalized auto-correlation value of 0.518 occurs at $4.8 \mathrm{~m}$. Based on the fact that the range bin with the peak auto-correlation value changes as the target is moved across each range bin, it can be concluded that the phase-coded-linear-FM signal has a resolution of $2.4 \mathrm{~m}$. From table 5.1, the peak auto-correlation value for the linearFM waveform occurs at $4.8 \mathrm{~m}$ (ignoring values at $2.4 \mathrm{~m}$ ) with a peak normalized autocorrelation value of 0.987 . From table 5.1 , if the target is at $7.2 \mathrm{~m}$ and the auto-correlation values at the $2.4 \mathrm{~m}$ range bin are ignored, a peak normalized auto-correlation value of 0.545 occurs at $7.2 \mathrm{~m}$. It can again be concluded that the linear-FM signal has a resolution of $2.4 \mathrm{~m}$ since the range bin with peak auto-correlation value changes as the target is moved across each range bin. For the phase-coded waveform, the peak normalized auto-correlation value is 0.819 and it occurs when the target is $4.8 \mathrm{~m}$ from the Radar. However, for subsequent pulses, as the target distance from the radar is increased, the range bin with the peak normalized 
auto-correlation value remains at $4.8 \mathrm{~m}$ as can be observed from table 5.1. This means that the phase-modulated signal has a range resolution which is greater than $9.6 \mathrm{~m}$.

As calculated in Chapter 5, the rotating fan generates Doppler shifts at $55.728 \mathrm{~Hz}$, 111.46Hz, 69.295 and 138.59 and serial Doppler processing yields Doppler shifts which differ from the calculated values by $3.872 \mathrm{~Hz}, 7.74 \mathrm{~Hz}, 6.21 \mathrm{~Hz}$ and $1.175 \mathrm{~Hz}$ respectively. The maximum deviation is less than the FFT resolution bin of $8.5 \mathrm{~Hz}$ implying an acceptable accuracy for experimentally determined values. Finer accuracy could be achieved by increasing the FFT length. Parallel processing only yields Doppler shifts at the edge frequencies of $111.46 \mathrm{~Hz}$ and 138.59 with discrepancies of $3.872 \mathrm{~Hz}$ and $10.41 \mathrm{~Hz}$ respectively. The discrepancies are again within the $14.9 \mathrm{~Hz}$ frequency resolution for parallel Doppler processing thus the Doppler accuracy is acceptable. Better accuracy can again be improved by increasing the FFT length and number of pulses required for processing. The inability of parallel Doppler processing to detect Doppler shifts resulting from the center of the fan blades is as a result of the number of processed pulses. Increasing the number of pulses will increase the FFT length and consequently the CNR at the Doppler frequency. Table 5.2 shows the difference in CNR at the Doppler frequencies as obtained by the serial and parallel processes. For simulated values, CNR is independent of the Doppler frequency. The serial process yields a simulated CNR of $55 \mathrm{~dB}$ while the parallel process yields a simulated CNR of $27 \mathrm{~dB}$. This implies an improvement of $28 \mathrm{~dB}$ of the serial process over the parallel for the same processing speed. Table 5.2 shows the tested CNR improvement ratios for all Doppler frequencies. The tested serial CNR at DC is 4dB less than the simulated value and $17 \mathrm{~dB}$ more than the parallel CNR. The tested serial CNR at Doppler frequencies due to 
the center of the fan $(55.73 \mathrm{~Hz}$ and $19.296 \mathrm{~Hz})$ is $38 \mathrm{~dB}$ which is about $17 \mathrm{~dB}$ below the simulated value and about $20 \mathrm{~dB}$ above the tested parallel CNR. The $20 \mathrm{~dB}$ improvement factor between the serial and parallel process is reasonably close to the simulated $28 \mathrm{~dB}$ value and shows that serial processing yield a higher CNR than parallel processes for comparable processing time. The difference between the simulated and tested CNR is because real channel effects such as target cross-section, and actual antenna gain tend to increase the signal attenuation. At edge Doppler frequencies of $111.46 \mathrm{~Hz}$ and $138.59 \mathrm{~Hz}$, the parallel to serial improvement factors are 9.83 and 5.33 respectively which are much lower than the simulated $28 \mathrm{~dB}$ value. However, the improvement factor still indicates that there is a significant CNR benefit in using serial instead of parallel Doppler processing.

\subsection{Conclusion}

The main objective of this thesis was to come up with a Radar waveform which requires low transmitter power, possesses fine range resolution and rejects ambiguous targets. Another objective was also to examine the effect of parallel and serial Doppler processing on the carrier to noise ratio. From Radar theory, it has been shown that minimum transmitter power is obtained from continuous waveforms, however to achieve fine range resolution, pulse compression is required. Chapter 2 of this thesis then examines frequency and phase modulated waveforms as two types of compression schemes. The phase-modulated waveform is theoretically shown to possess the ambiguous target rejection property while the frequency modulated signal has the finer resolution of the two. By convolving the frequency 
and phase modulated signals, a new waveform is obtained which has the fine resolution of the FM signal and ambiguous target rejection property of the PM signal. Simulations are then carried out (based on the proposed waveform) to test the ability of the receiver processor to detect the range of a target and its speed in the presence of clutter, path loss, multipath, target cross-section and AWGN. Target range is obtained by Auto-Correlation and ambiguous target rejection is done by Cross-Correlation. Figure 5.6 verifies the resolution property of the proposed waveform while figure 3.4 verifies the cross-correlation property. Simulation also shows a $28 \mathrm{~dB}$ benefit in CNR of serial Doppler processing compared to parallel Doppler processing for comparable processing times. Experimental evaluation of the proposed waveform is done by using the D-TA 2300 and D-TA 3290 which are IF and RF processors respectively. The power benefit obtained by the pulse compression waveform is expressed in terms of the maximum achievable range with and without pulse compression. Given the hardware restrictions, the calculated maximum range without pulse compression is $11.45 \mathrm{~m}$ while the calculated maximum range with pulse compression is 64.76 . From tested results shown in figure 5.6, a maximum range of $40 \mathrm{~m}$ can be observed from the auto-correlation plot. This implies that for the same maximum range, a system which does not utilize pulse compression requires greater transmitter power than a system that does. For range resolution testing, a rectangular metal object is placed at various range bins and the Auto-Correlation responses of all 3 types of waveforms are obtained. Figure 5.6 to 5.8 show that the phasecoded-linear-FM signal has the same resolution as the linear FM signal $-2.4 \mathrm{~m}$ while the phase modulated signal has a range resolution greater than $9.6 \mathrm{~m}$. It was not possible to verify ambiguous target rejection experimentally because for ambiguous targets to be detected, the 
maximum range has to be greater than the unambiguous range. This condition was not fulfilled in this case because the transmitted power was too small to detect any target over the unambiguous range. Doppler processing is done with a rotating fan as the target. The Doppler velocities are calculated for rotating speeds of 1220rpm and $1517 \mathrm{rpm}$ and compared with those obtained from the received echo. Serial Doppler processing yields CNRs at the processed Doppler shifts which are about 20dB higher than those obtained from parallel Doppler processing as shown in table 5.2. This indicates that for comparable processing times, serial Doppler processing is better than parallel Doppler processing as the obtained CNR at the Doppler frequency is considerably higher. This thesis has thus shown by experimental evaluation that pulse compression in the presence of a continuous waveform requires low transmitter power and achieves high resolution. Pulse compression also allows for high CNRs when processing for Doppler as auto-correlation is used for echo compression and this increases the SNR of the return signal.

Contributions made in this thesis include the derivation, simulation and testing of a new waveform which rejects ambiguous targets and possesses high resolution. The resolution property was verified through testing but the ambiguous target rejection property was not because of transmitter power restrictions. Another significant contribution is the utilization of auto-correlation values to obtain the Doppler shift in serial Doppler processing and the resulting CNR improvement over parallel Doppler processes.

Further work in this research can be carried out in the waveform design by increasing the peak to side-lobe ratio of the auto-correlation response. This can be done by utilizing poly-phase codes instead of PN sequences as they possess a better peak to side-lobe ratio. 
Side-lobe reduction in the linear FM signal can also be done by frequency weighting but at the expense of lower range resolution. The demonstrated waveform in this thesis can thus be improved by developing a coding scheme which has better cross-correlation and side-lobe properties than PN sequences. Future work can also includes testing of the ambiguous target rejection property of the waveform by utilizing more transmit power. Finally, the Doppler test demonstrated in this research could be more definitive if the transmitted signal is focused perpendicular to the edge of the fan blades such that a single Doppler frequency is obtained. 


\section{Appendix A \\ Antenna Measurements}

Figure 6.1: Elevation plane Gain measurement for the $\mathrm{H}-1734$ antenna at $1.5 \mathrm{GHz}$

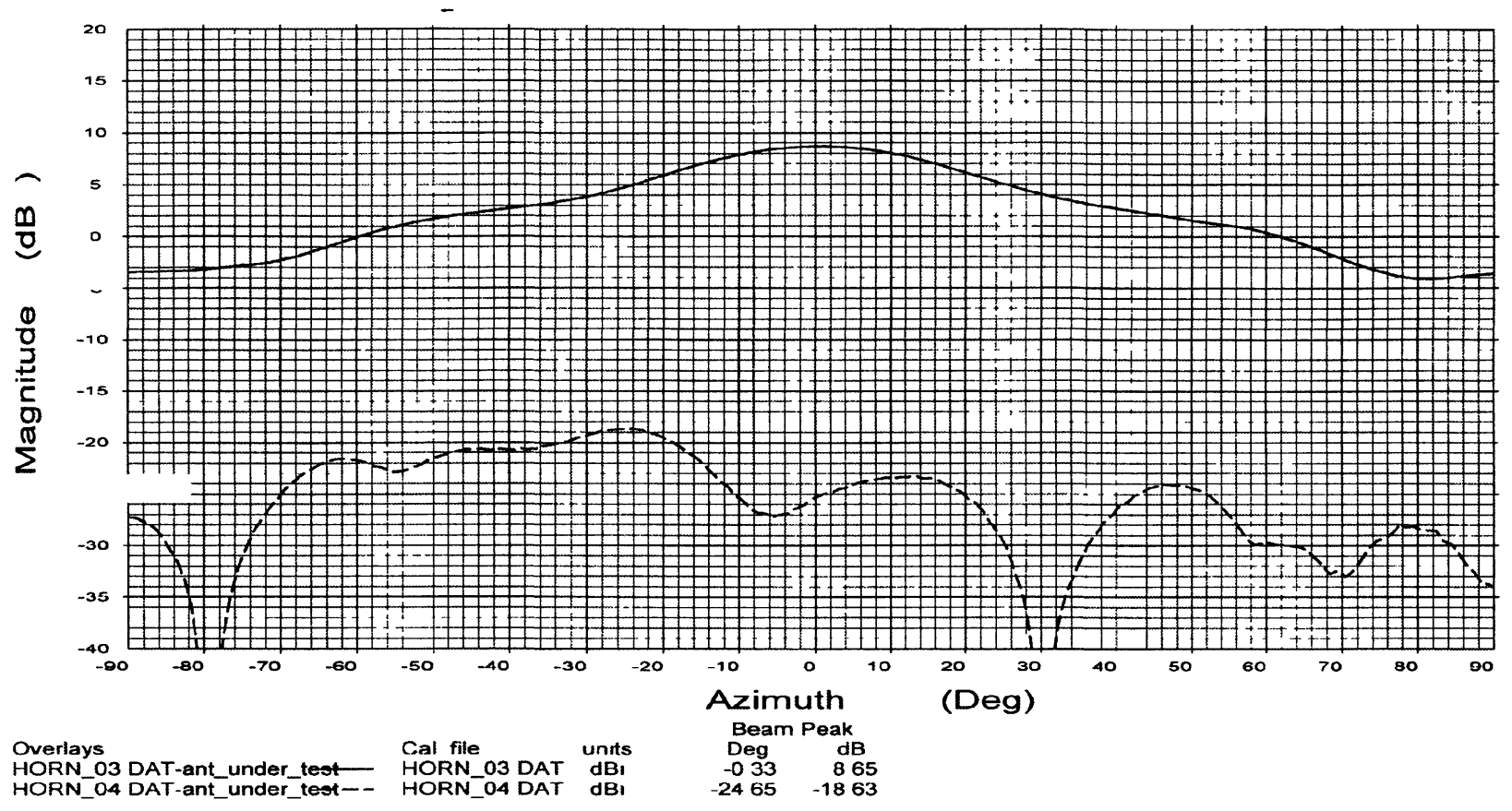

Figure 6.2: Azimuth plane Gain measurement for the $\mathrm{H}-1734$ antenna at $1.5 \mathrm{GHz}$

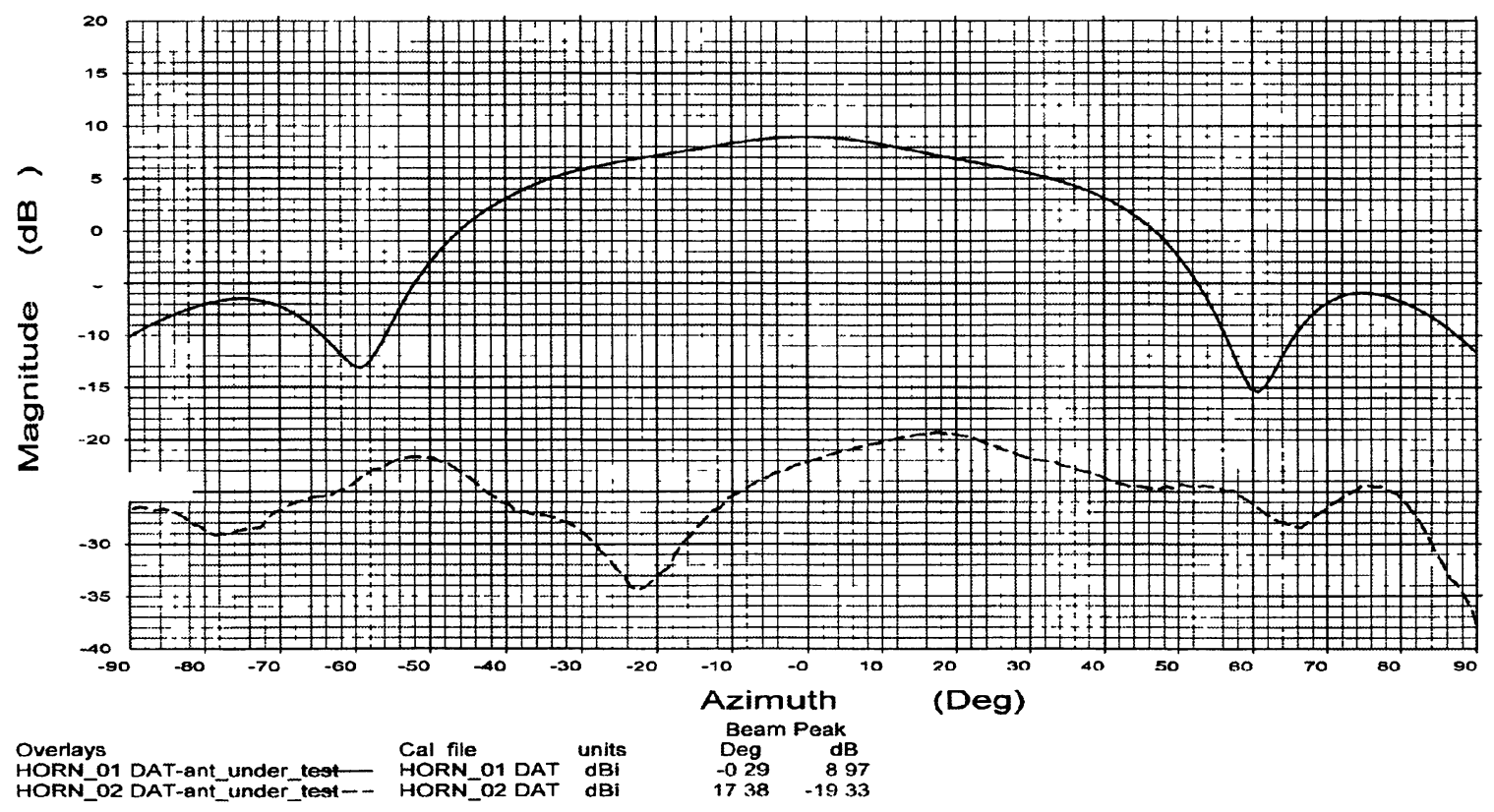




\section{Bibliography}

[1] Merrill I. Skolnik, Dean D. Howard, Radar Handbook, 2nd ed., McGraw-Hill, 1990, pp. $18.46-18.50$

[2] Merrill I. Skolnik, David J. Murrow, Radar Handbook, 2nd ed., McGraw-Hill, 1990, pp.20.33-20.39

[3] Merrill I. Skolnik, Radar Handbook, 2nd ed., McGraw-Hill, 1990, pp.1.1 -1.13

[4] Blake, L. V,: “A Guide to Basic Pulse-Radar Maximum-Range Calculation Part-1”, Naval Research Lab. Washington D.C. 20362, December 23rd 1969

[5] Merrill I. Skolnik, Lamont V. Blake, Radar Handbook, 2nd ed., McGraw-Hill, 1990, pp. $2.20-2.26$

[6] Merrill I. Skolnik, Edward C. Farnett and George H. Stevens, Radar Handbook, 2nd ed., McGraw-Hill, 1990, pp.10.15-10.26

[7] Merrill I. Skolnik, William K. Saunders, Radar Handbook, 2nd ed., McGraw-Hill, 1990, pp.14.21-14.28

[8] G. L. Stuber, Principles of Mobile Communication, 2nd ed., Kluwer, Dordrecht, 2001, Chapter 9.2

[9] Andrea Goldsmith, Wireless Communications, 1st ed., Cambridge University Press, 2005, pp. $403-417$ 
[10] Merrill I. Skolnik; William H. Long, David H. Mooney and William A. Skillman, Radar Handbook, 2nd ed., McGraw-Hill, 1990, pp.17.2-17.18

[11] Merrill I. Skolnik, Lewis B. Wetzel, Radā̄ Handbook, 2nd ed., McGraw-Hill, 1990, pp.13.8-13.23

[12] http://www.windfinder.com/wind/windspeed.htm

[13] Rodger E. Ziemer and Roger L. Peterson, Digital Communications and spread Spectrum Systems, Macmillan Publishing, 1985, pp.333-338

[14] Matteo Frigo and Steven G. Johnson, "The Design and Implementation of FFTW3", Proceeding of the IEEE, vol. 93, no. 2, February 2005, pp. 216-231.

[15] Cobham Sensor Systems, "Spiral Antennas", Retrieved May 4th, 2010, from https://www.cobham.com/media/101028/Spiral\%20Catalog\%20-\%20Lansdale.pdf 